\title{
Relational Concept Knowledge in a Social Network
}

\author{
by \\ Shaun McQuaker \\ A thesis submitted to \\ the Faculty of Graduate Studies and Research \\ in partial fulfillment of the requirements for the degree of \\ Master of Computer Science
}

\author{
Ottawa-Carleton Institute for Computer Science \\ School of Computer Science \\ Carleton University \\ Ottawa, Canada
}

May, 2007

Copyright (C) 2007 Shaun McQuaker 


$\begin{array}{ll}\begin{array}{l}\text { Library and } \\ \text { Archives Canada }\end{array} & \begin{array}{l}\text { Bibliothèque et } \\ \text { Archives Canada }\end{array} \\ \begin{array}{l}\text { Published Heritage } \\ \text { Branch }\end{array} & \begin{array}{l}\text { Direction du } \\ \text { Patrimoine de l'édition }\end{array} \\ \begin{array}{l}\text { 395 Wellington Street } \\ \text { Ottawa ON K1A 0N4 }\end{array} & \begin{array}{l}\text { 395, rue Wellington } \\ \text { Ottana ON K1A ON4 } \\ \text { Canada Oa }\end{array}\end{array}$

Your file Votre référence ISBN: 978-0-494-33682-3 Ourfile Notre référence ISBN: 978-0-494-33682-3

NOTICE:

The author has granted a nonexclusive license allowing Library and Archives Canada to reproduce, publish, archive, preserve, conserve, communicate to the public by telecommunication or on the Internet, loan, distribute and sell theses worldwide, for commercial or noncommercial purposes, in microform, paper, electronic and/or any other formats.

The author retains copyright ownership and moral rights in this thesis. Neither the thesis nor substantial extracts from it may be printed or otherwise reproduced without the author's permission.
AVIS:

L'auteur a accordé une licence non exclusive permettant à la Bibliothèque et Archives Canada de reproduire, publier, archiver, sauvegarder, conserver, transmettre au public par télécommunication ou par l'Internet, prêter, distribuer et vendre des thèses partout dans le monde, à des fins commerciales ou autres, sur support microforme, papier, électronique et/ou autres formats.

L'auteur conserve la propriété du droit d'auteur et des droits moraux qui protège cette thèse. $\mathrm{Ni}$ la thèse ni des extraits substantiels de celle-ci ne doivent être imprimés ou autrement reproduits sans son autorisation.
In compliance with the Canadian

Privacy Act some supporting forms may have been removed from this thesis.

While these forms may be included in the document page count, their removal does not represent any loss of content from the thesis.
Conformément à la loi canadienne sur la protection de la vie privée, quelques formulaires secondaires ont été enlevés de cette thèse.

Bien que ces formulaires aient inclus dans la pagination, il n'y aura aucun contenu manquant. 


\section{Abstract}

This thesis deals with the topic of peer-to-peer referral systems and the policies that allow for the emergence of efficient retrieval of requested information. In an agentbased peer-to-peer network, member agents are capable of giving and following referrals to each other. This results in the emergence of communities where agents directly interact with other neighbouring agents that supply the required service or will refer the right source. The notion of referral networks, as presented in the work of Singh and Yolum [1], and their application to knowledge management, lacks two fundamental aspects; the relation of concepts within a domain, and the ability of an agent to dynamically change their interests based on suggestions in the form of concept relations. This thesis introduces the concept of an "oracle" agent, which is an agent with relational concept knowledge that can supply suggestions to a querying agent on how to adapt their interests. Additionally the notion of health and localized trust automata are used to aid agents in discriminating useful pieces of concept knowledge. These new features allow agents to search in new ways so as to achieve superior results and as a consequence outperform agents in a traditional referral network. The thesis presents simulation results that confirm this hypothesis. 


\section{Acknowledgements}

First and foremost I would like to thank Tony White, my thesis supervisor for introducing me to referral networks and framing the initial problem that inspired this thesis. Without the tireless support and affirmations from Tony this thesis would never have been completed. I would also like to thank Tony for periodically checking his email spam folder for one of my many emails.

I cannot escape thanking Pinar Yolum and Munindar Singh for providing me a simulation test bed on which to build my thesis. Their work in this area is pioneering and without it I would have been hopelessly lost. Correspondence with Pinar was also helpful at key stages in this research.

My family and friends were continual sources of positive inspiration and encouragement, and have my deepest gratitude for standing by my side throughout this process. 


\section{Table of Contents}

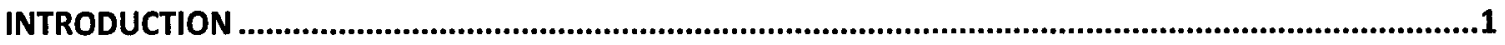

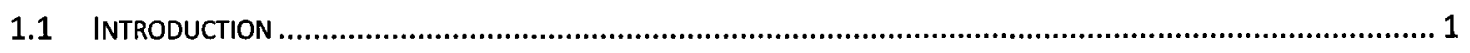

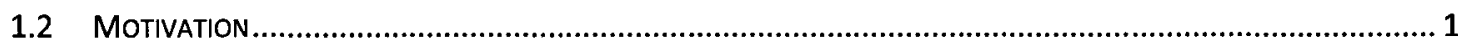

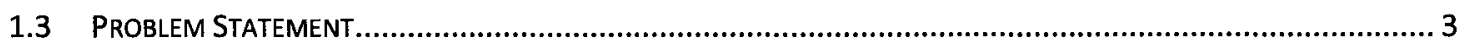

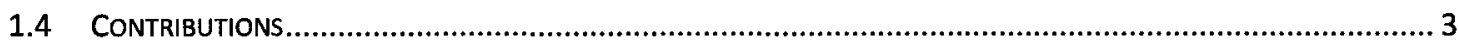

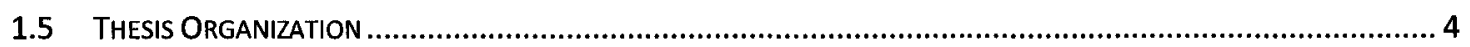

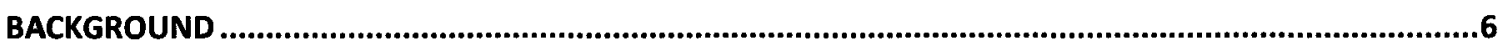

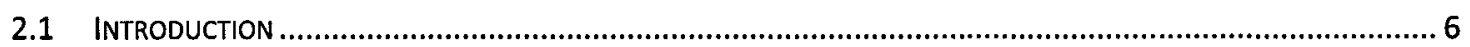

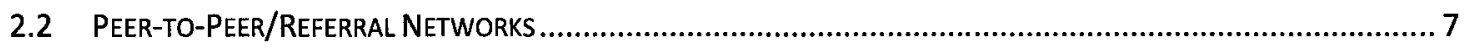

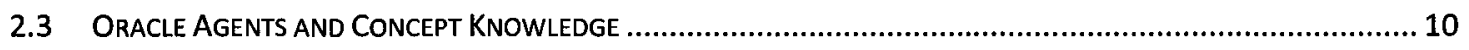

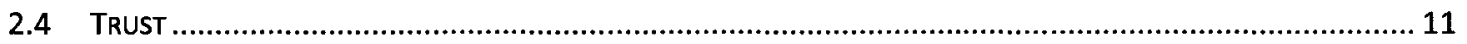

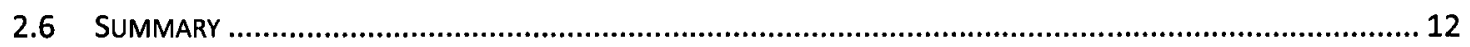

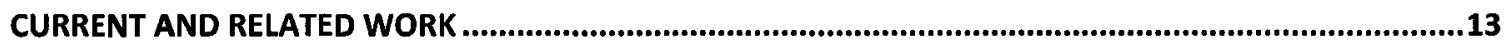

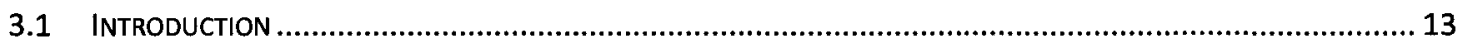

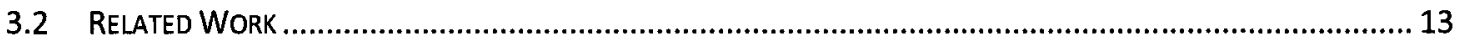

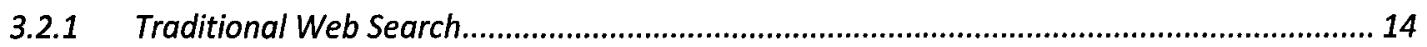

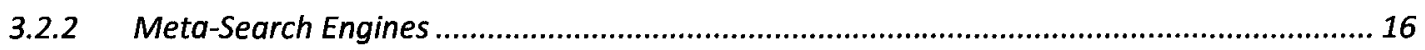

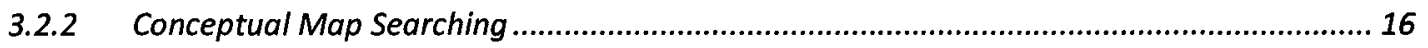

3.2.3 Ontologies and Recommender Systems......................................................................... 18

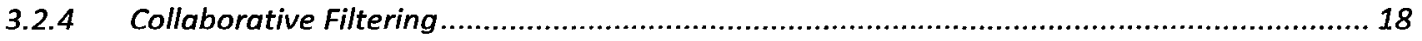

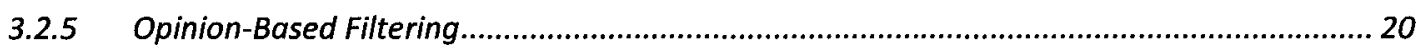

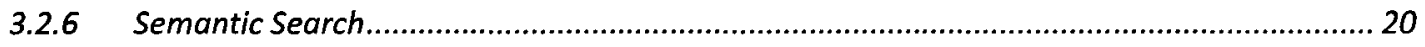

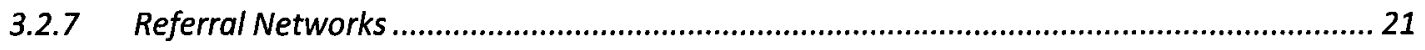

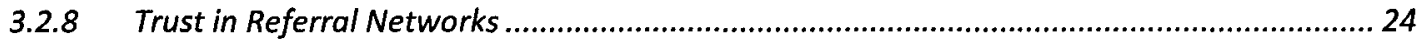


3.2.9 Summary

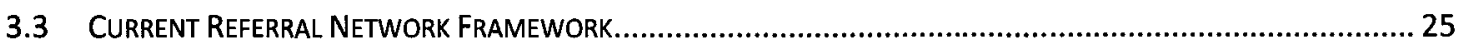

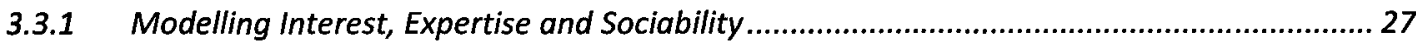

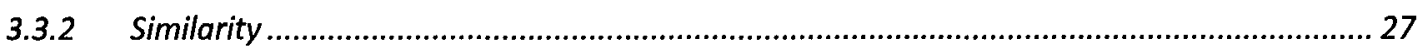

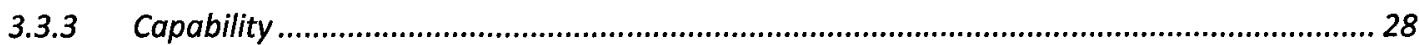

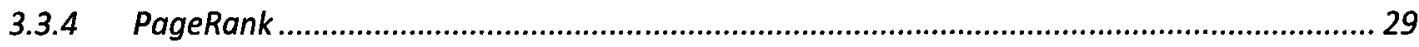

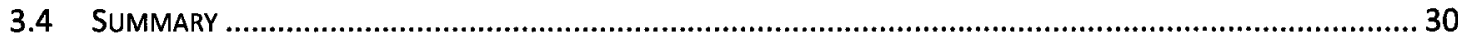

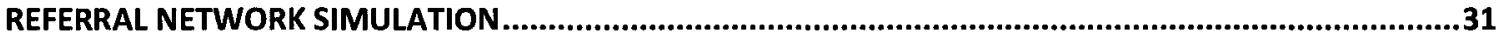

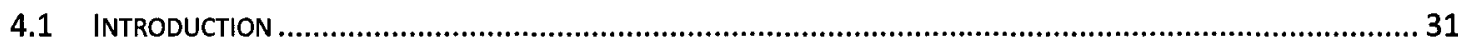

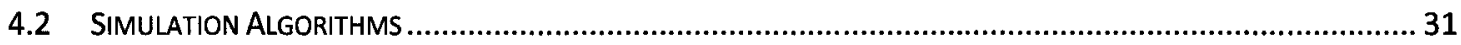

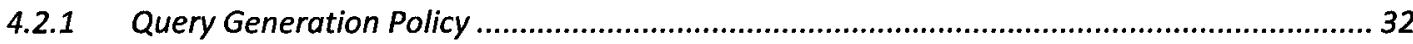

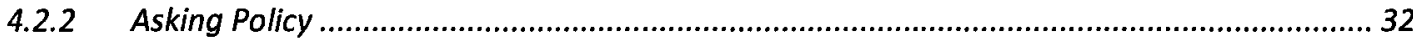

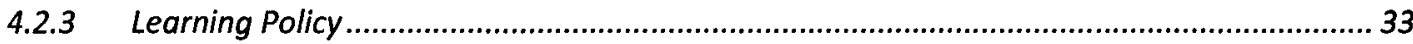

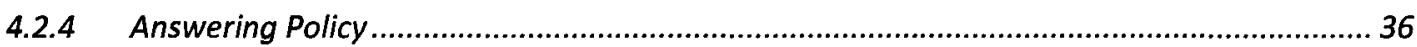

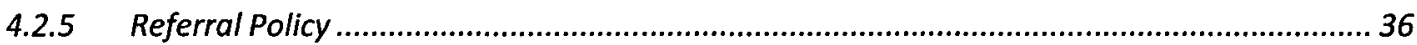

4.2.6 Neighbour Selection Policy.......................................................................................... 36

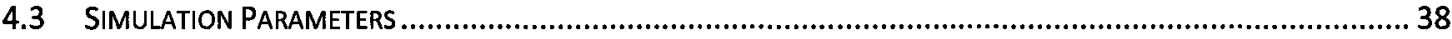

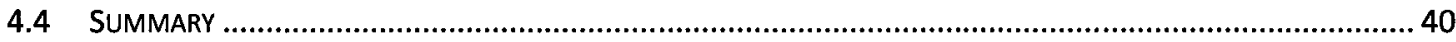

MEASURING PERFORMANCE OF REFERRAL NETWORKS ...................................................................41

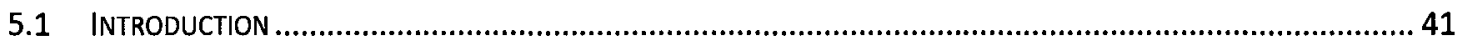

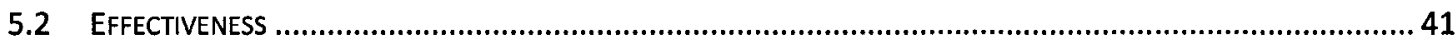

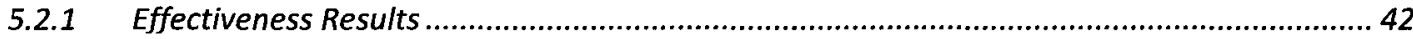

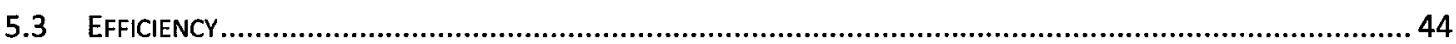

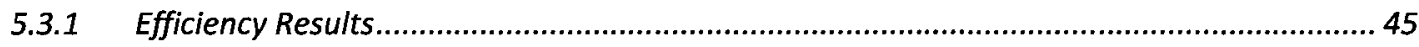

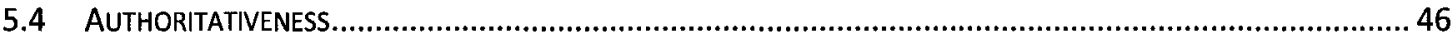

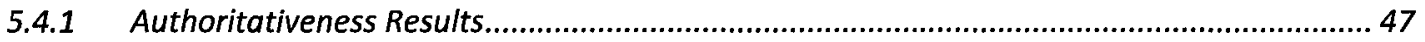

vi

Reproduced with permission of the copyright owner. Further reproduction prohibited without permission. 


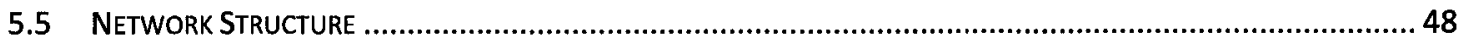

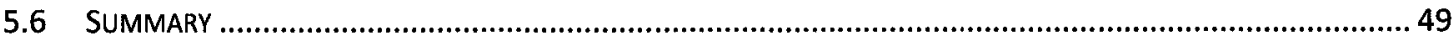

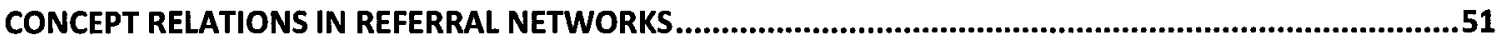

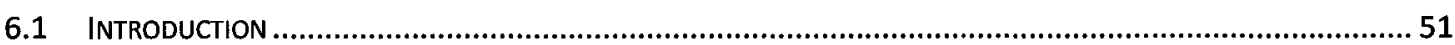

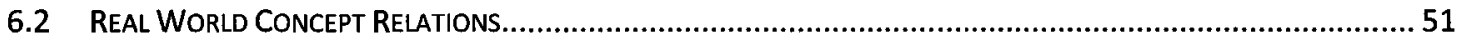

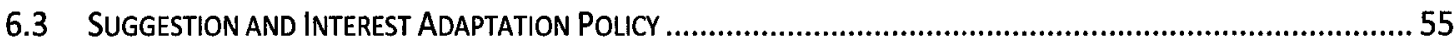

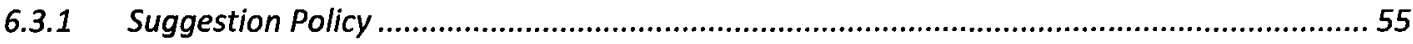

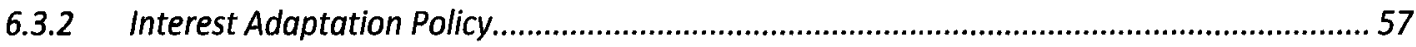

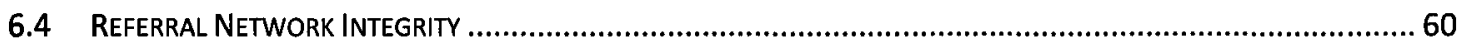

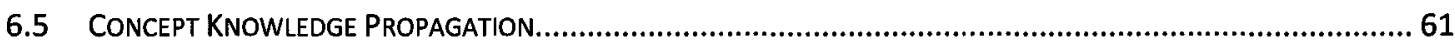

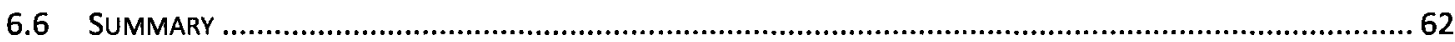

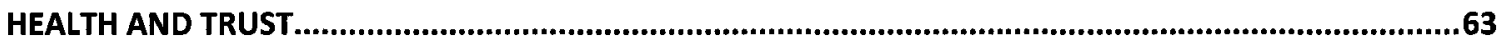

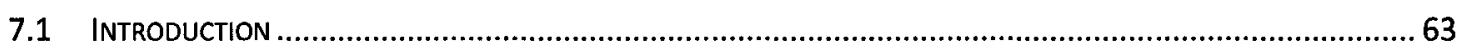

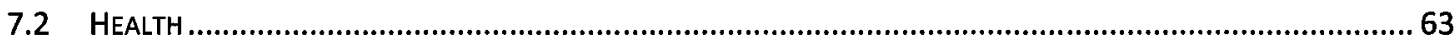

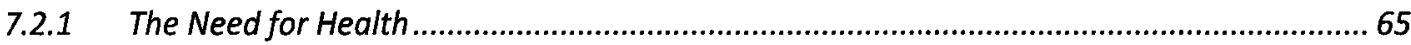

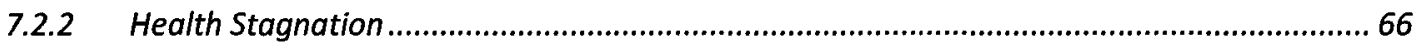

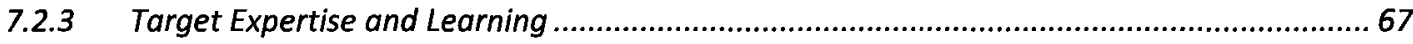

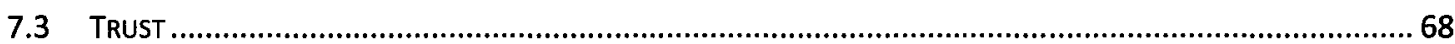

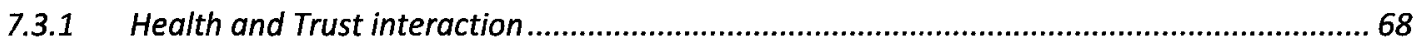

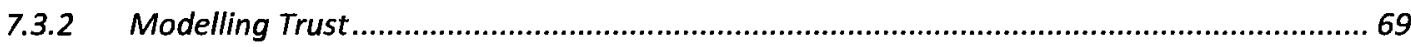

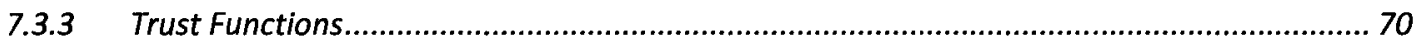

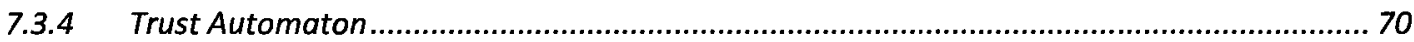

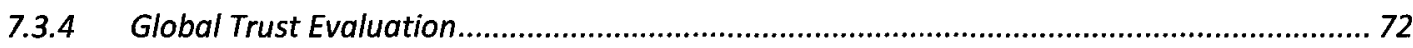

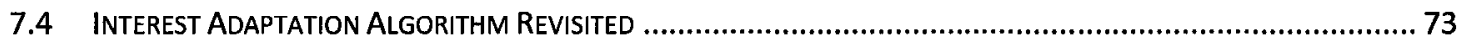

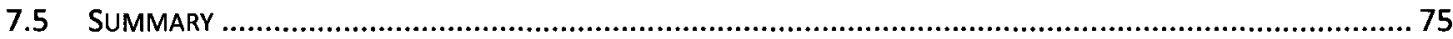

vii

Reproduced with permission of the copyright owner. Further reproduction prohibited without permission. 


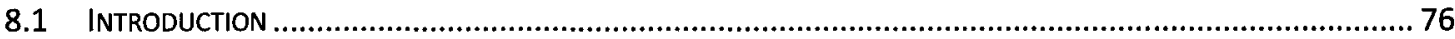

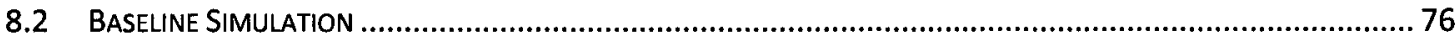

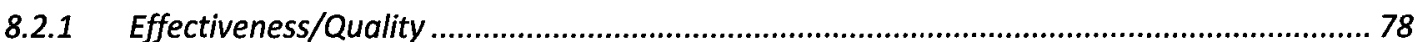

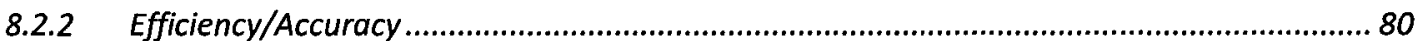

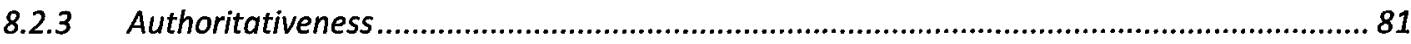

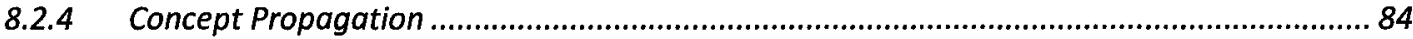

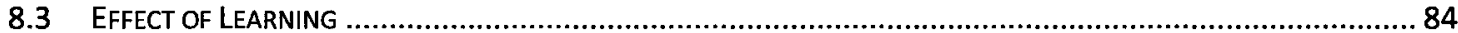

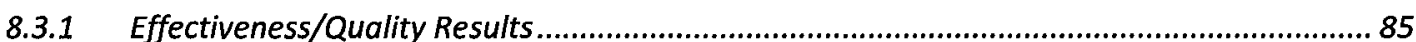

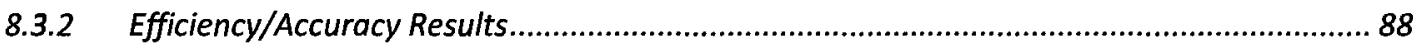

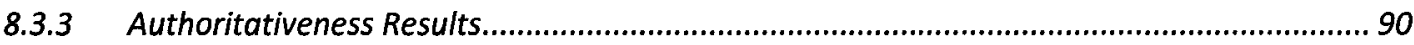

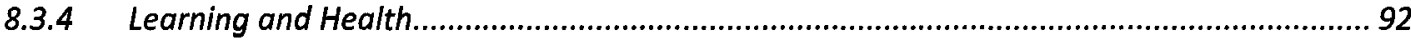

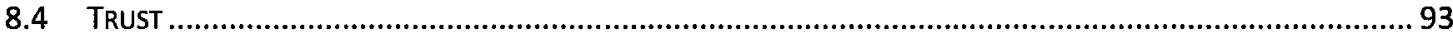

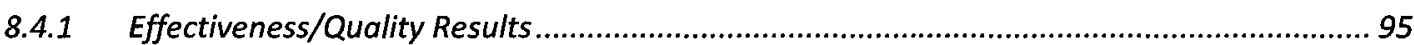

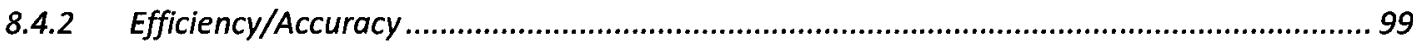

8.4.3 Oracle Trust and Agent Health....................................................................... 101

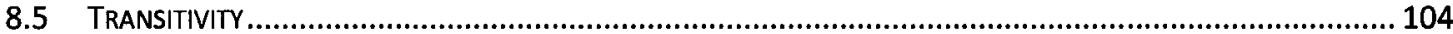

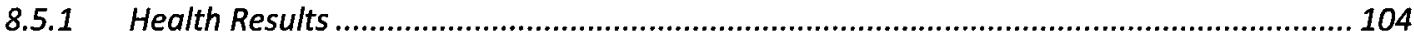

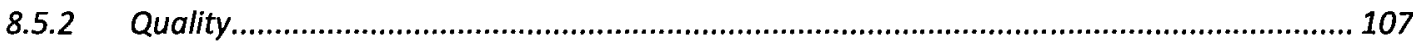

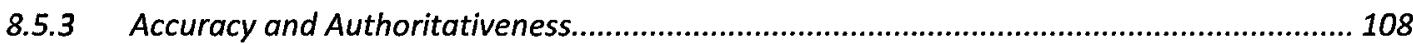

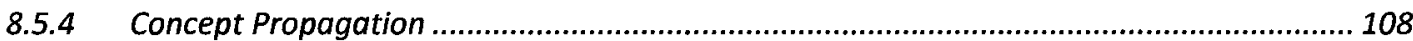

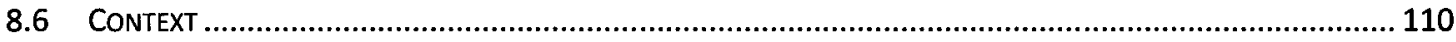

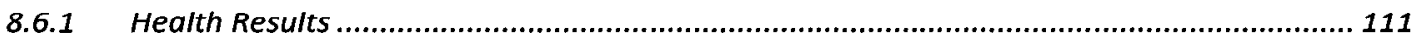

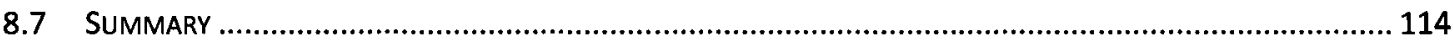

CONCLUSIONS AND FUTURE WORK.....................................................................................115

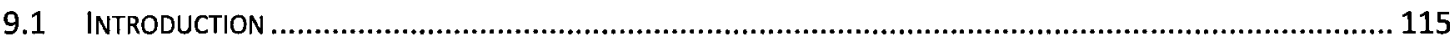

viii

Reproduced with permission of the copyright owner. Further reproduction prohibited without permission. 


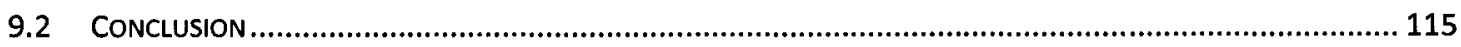

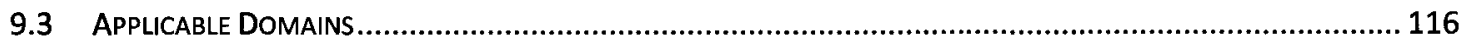

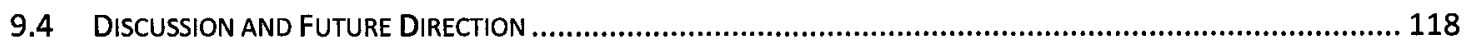

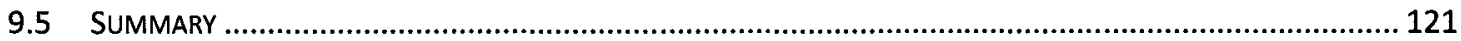

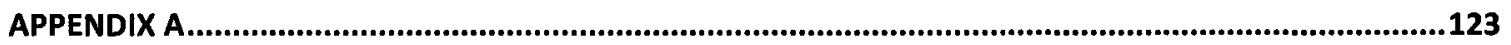

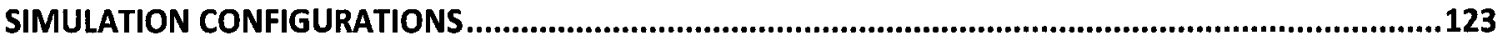

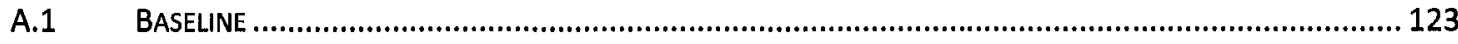

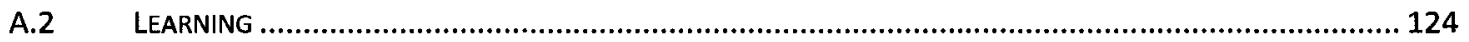

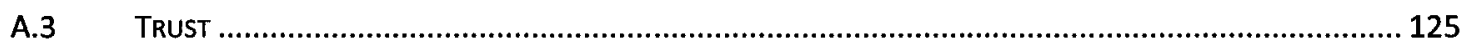

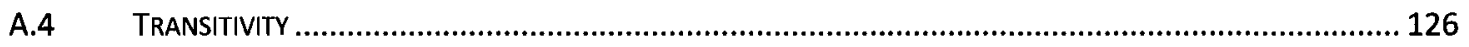

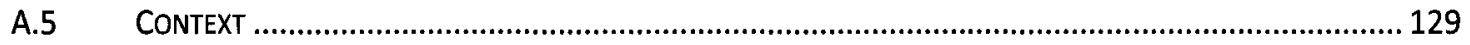




\section{List of Figures}

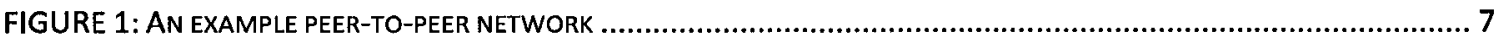

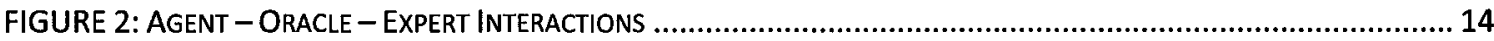

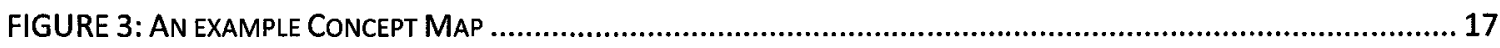

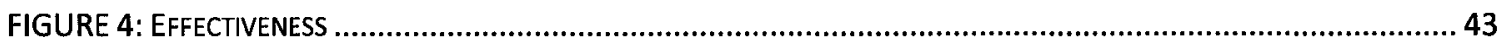

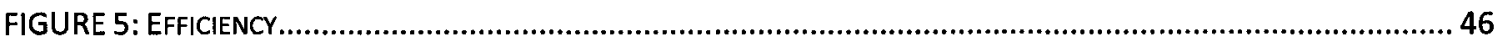

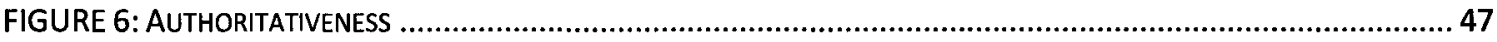

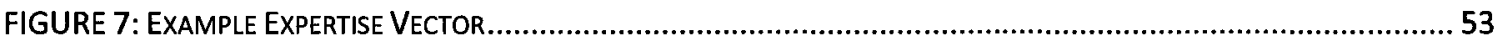

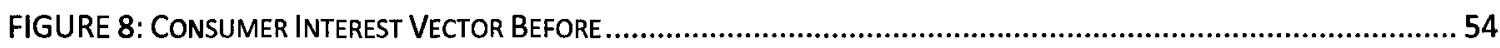

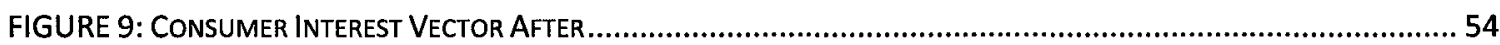

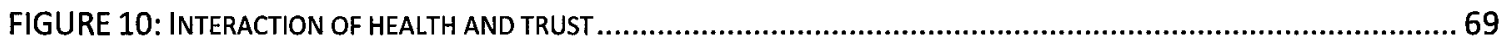

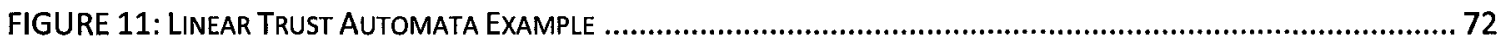

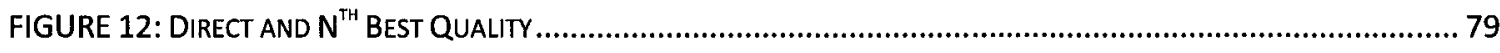

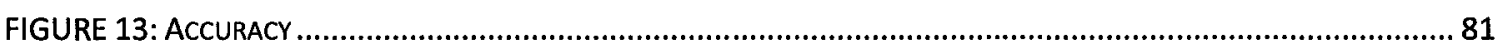

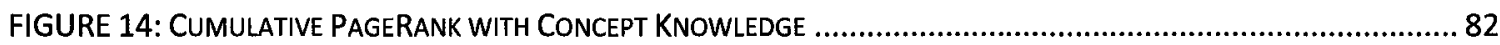

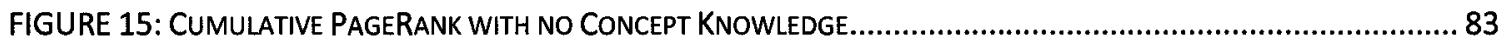

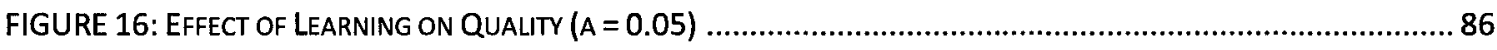

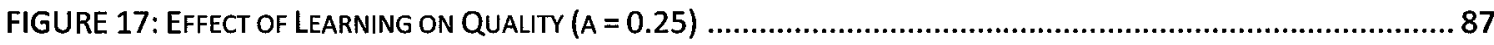

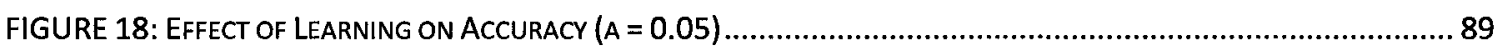

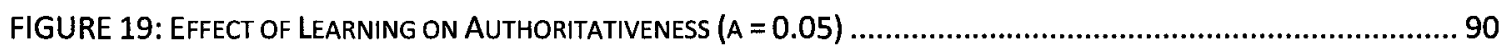

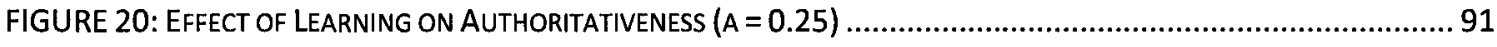

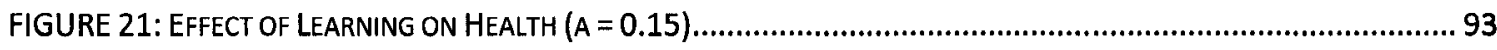

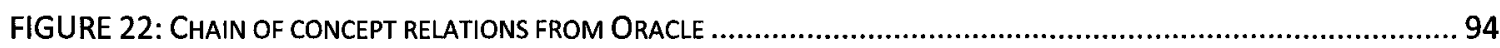

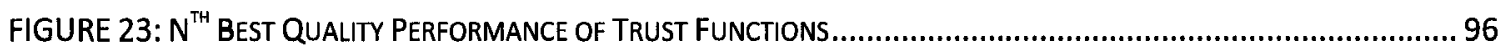

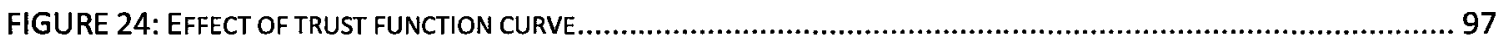

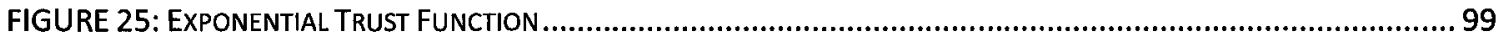


FIGURE 26: ACCURACY PERFORMANCE OF TRUST FunCTIONS.

FIGURE 27: AVERAGE HEALTH AND VARIANCE

FIGURE 28: HEALTH OVER TIME.

FIGURE 29: INFRASEARCH OVERVIEW 


\section{List of Equations}

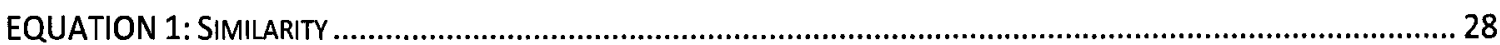

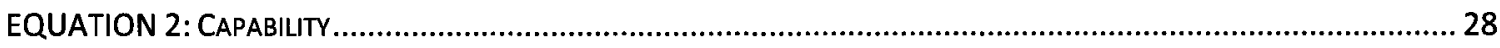

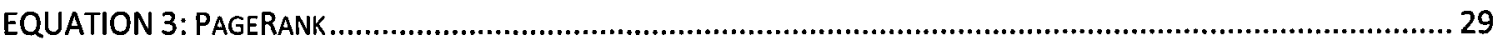

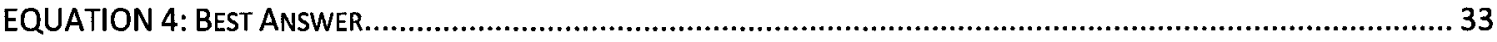

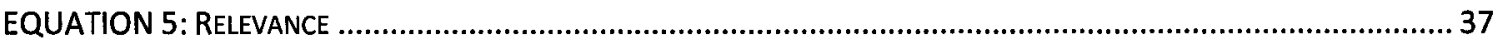

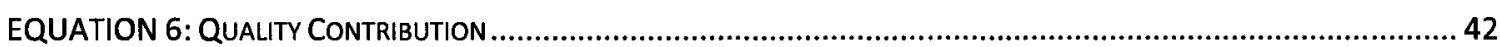

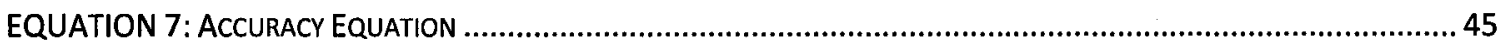

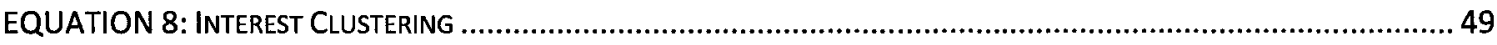

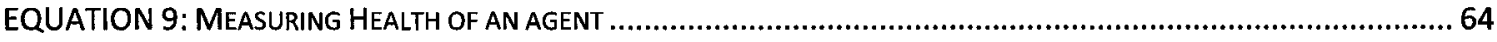

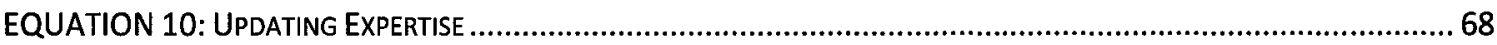

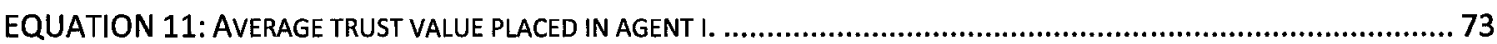




\section{List of Algorithms}

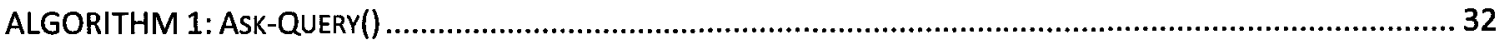

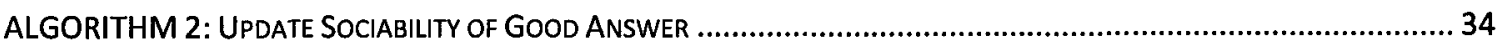

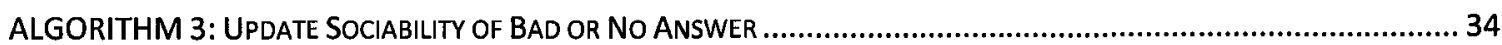

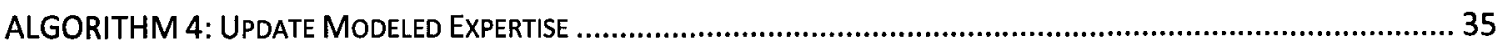

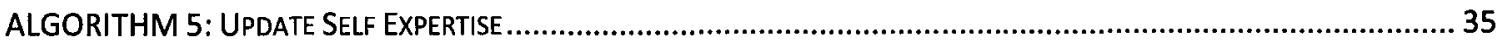

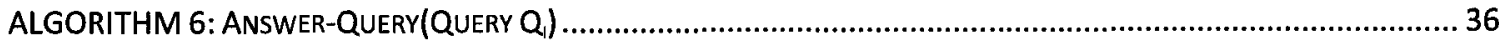

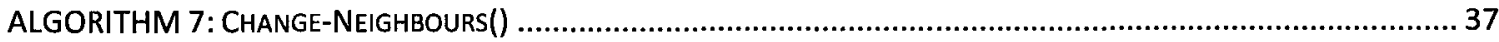

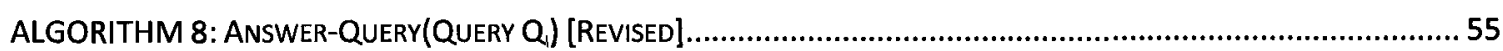

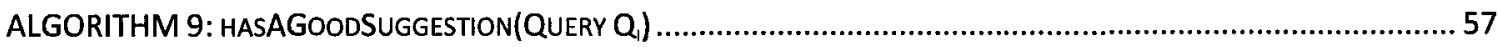

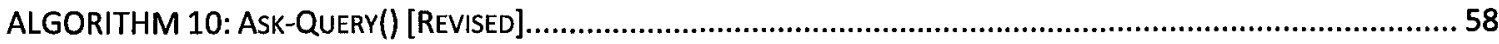

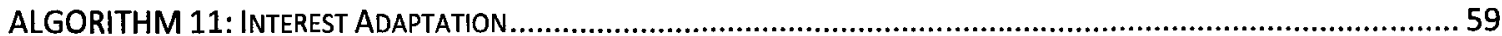

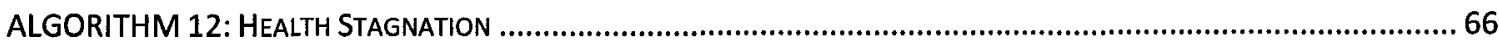

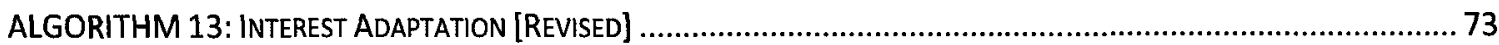




\section{Chapter 1}

\section{Introduction}

\subsection{Introduction}

In day-to-day life, social networks within human communities are created and changed constantly. Social networks as we know them in real-life consist of groups of connected people which have fashioned some form of a model that enables them to interact with each other in unique and mutually advantageous ways. The diversity of our social networks is not only essential to our survival but act as an aide in our psychological and educational development. Social networks also exist in the online world, allowing people to connect and learn from each other in ways never before possible. The use of social networks to form communities and thus enable a more personalized and intuitive search experience is becoming a major contribution to the online world.

\subsection{Motivation}

Concepts derived from real-life social networks have provided powerful computational tools in a variety of areas in computer science. From providing an architecture for social networking sites such as Facebook.com ${ }^{1}$ to managing institutional knowledge in medical and corporate arenas [2], social networks are

${ }^{1}$ http://www.facebook.com 
gaining traction as a useful tool for controlling and disseminating knowledge and connecting people. The application of real-life social networking concepts to the online world is becoming ever more important as the Web is becoming an extension of our every-day lives. There are increasing pressures not to access but rather to efficiently find useful information. The success of companies like Google and Yahoo are a testament to how important searching is in our everyday lives.

As the Internet continues to grow it is increasingly hard to find useful information using traditional search methods. Existing keyword-based search engines are "becoming ineffective due to the rapid growth and changes in the contents of the World Wide Web" [3]. This is due to the fact that current keyword-based searching allows little to no contextual data to be given; it cannot distinguish a musical keyboard from the keyboard used to write this thesis. For instance, searching for the word "matrix" on ${ }^{2}$ Google "yields 4,380,000 results and included in the first 20 are references to a movie, the mathematical concept, four different companies, a data center, a monastery, a display device, a hair care product and an on-line community." [4] Additionally, current search engines do little to provide sources of conceptually related knowledge. For instance, they do not suggest searching for the term computer keyboard if you enter "keyboard" as your search keyword.

Information retrieval researchers have suggested that the process of seeking information has always been a social process [5], thus applying social networking

\footnotetext{
${ }^{2}$ http://www.google.com
} 
concepts to internet searching has the potential to alleviate some of the complexities of online searching by forming communities around sources of expertise; people, documentation, computer code, etc. Social communities or groups can then aid new as well as existing users in learning through the provision of useful information from well connected sources of expertise.

\subsection{Problem Statement}

Current social networking solutions in conjunction with the global reach of the Internet act as a great medium to connect people with pieces of useful information. However, current social networks must evolve methods that better mirror how we as humans model our world and the knowledge therein. Current work for modelling social networks with referral networks does not provide two essential ingredients that humans use in real-life social networks; concept knowledge and suggestions which propagate concept knowledge. The ability for referral networks to provide a mechanism for the dissemination of concept knowledge through suggestions and thus the ability to distinguish context is essential to reducing the complexity of searching through the vastness of knowledge available on the Internet today. This thesis, therefore, proposes solutions to the concept knowledge propagation problem within the scope of referral networks.

\subsection{Contributions}

This thesis builds on top of the existing work in the field of Referral Networks by extending how knowledge is maintained and modelled in those networks. The 
objective is to study the effect of relational concept knowledge on the quality, efficiency and authoritativeness of a referral system. Thus, how agents within a referral network with concept knowledge assist other agents in finding useful knowledge via suggestions is examined and several learning algorithms are proposed for concept propagation, learning, and evaluation. Additionally, localized concept knowledge trust is modelled using a localized trust automaton to aid in minimizing risk of using useless or malicious pieces of concept knowledge. The notion of agent health is also introduced as a reinforcement technique to drive evaluation of concept knowledge.

\subsection{Thesis Organization}

The remainder of the thesis is organized as follows: Chapter 2 provides essential background information on referral networks, trust and concept knowledge. Chapter 3 introduces current and related work on referral networks and explains some of the underlying concepts in the area. Chapter 4 outlines how a referral network is simulated, and reviews algorithms by Singh and Yolum. Chapter 5 outlines performance metrics for measuring referral networks. Chapter 6 introduces concept knowledge and how it can be applied to referral networks to improve the modelling of social networks. Chapter 7 establishes the notion of agent health and its role in localized trust for concept knowledge. Chapter 8 presents simulation results with referral networks employing relational concept knowledge and provides key insights into the improvements that the added knowledge 
provides. Finally, Chapter 9 gives conclusions and outlines potential future work in this expanding field. 


\section{Chapter 2}

\section{Background}

\subsection{Introduction}

An overview of the referral network architecture used to computationally model social networks is presented in this chapter. Additionally, the notion of oracle agents is introduced as the mechanism by which concept knowledge is propagated in a referral network and lastly trust is introduced as it plays a vital role in finding useful information in a referral network.

The popular adage "it's not what you know, but who you know" exists for a reason. People tend to use their personal social networks more when "faced with reduced institutional support." [6] Take the real-life example of being faced with a problem you cannot solve. Usually we turn to a trusted source or expert for advice on how to proceed with solving the problem at hand. If we do not know of such a person, we usually ask our friends if they know such a person, and could perhaps provide us with some initial contact. If our friends cannot help us or provide a referral they may be able to provide a suggestion as to what else we may research to find a solution. In this way we rely on our social network for three things, advice, referrals to experts, or suggestions on how to reframe our problem such that a solution may be more forthcoming. It is this reframing of the problem that is the focus of this 
thesis. Peer-to-peer referral networks can be used to computationally model these sets of interactions.

\subsection{Peer-to-Peer/Referral Networks}

A peer-to-peer network (P2P) is a decentralized network of nodes/agents that exchange information between each other. This network is formed "on top of-and independently from—the underlying physical computer (typically IP) network, and is thus referred to as an 'overlay' network." [7] File-sharing systems such as Limewire and Gnutella use the peer-to-peer architecture as an overlay peer-to-peer network to propagate queries (for a file) from one peer (agent) to another, thus allowing the exchange of files without the need for a centralized server. Figure 1 graphically depicts the structure of a P2P network, where nodes are the blue dots and the lines are connections to peers in the network.

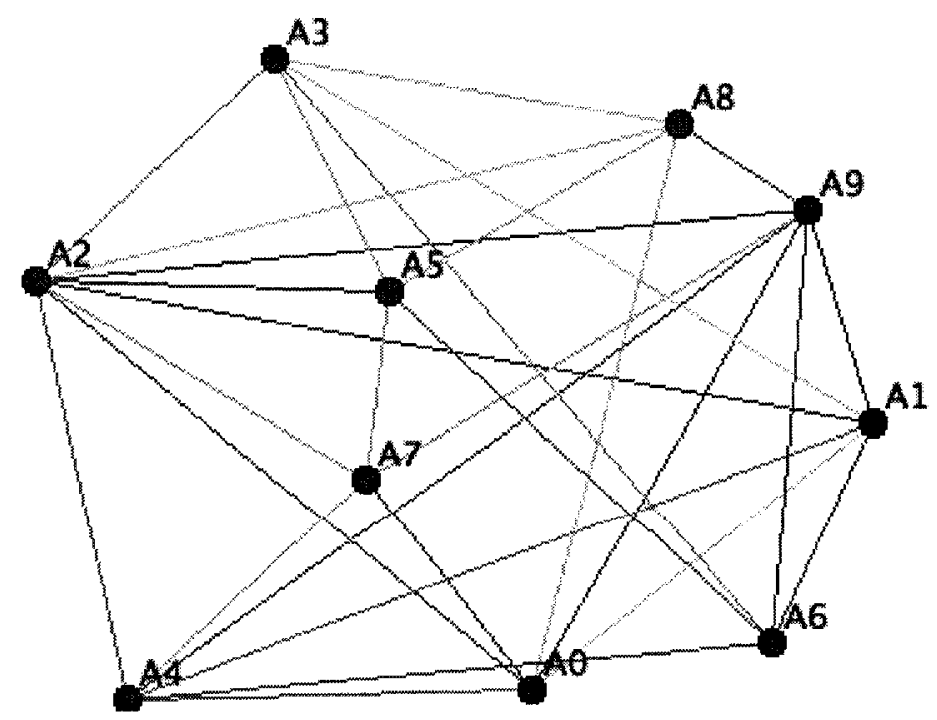

FIGURE 1: An example peer-to-peer network 
The motivation for using decentralized peer-to-peer networks is "their ability to function, scale, and self-organize in the presence of a highly transient population of nodes, network, and computer failures." [7] Freenet [8] is a self-organizing decentralized P2P network content management system meant to provide electronic freedom of speech through strong anonymity. As data is inserted into the network, it is replicated over several nodes, thus ensuring that if one node is removed from the network data access is still maintained. Additionally most P2P networks do not require a central server to coordinate the activities and connectivity of nodes within the network; they are by nature decentralized. As there is no single point of failure, the resilience of P2P networks and their low administration needs make them very appealing to any sort of content distribution system.

Since a peer-to-peer network is never completely connected, communication to peers who are not direct neighbours must be done through existing neighbours. Thus the "topology, structure, and degree of centralization of the overlay network, and the routing and location mechanisms it employs for messages and content are crucial to the operation of the system, as they affect its fault tolerance, self maintainability, adaptability to failures, performance, scalability, and security." [7] For instance, the distribution of content is a problem faced by any peer-to-peer network. More "popular content is likely to be available at several peers and any peer searching for it is likely to find the same thing, but if a peer is looking for rare 
data shared by only a few other peers, then it is highly unlikely that search will be successful." [9]

The referral-based architecture of Singh and Yolum [10] can also be thought of as a peer-to-peer overlay network. Consumer agents representing the interests of their real-life operators attempt to find provider agents in the network via a system of referrals. Agents pose questions to direct neighbours which can either answer these questions if they have sufficient expertise or refer a neighbour of their own that could possibly answer the question. Based on previous interactions agents build local models of their neighbours thus allowing better referrals to be given. Once a consumer has found a provider in the network, it can judge the quality of service from the provider as well as the quality of referrals it received for the provider. As in peer-to-peer networks, considerations such as the number of neighbours, the policy for changing and referring neighbours and answering queries determines the structure and quality of a referral network.

Similar to their real-life counterparts, agents in a referral network are autonomous and heterogeneous. That is, they are autonomous in the sense that they can "decide what actions they want to perform, with whom they want to interact, or how they want to carry out their tasks." [10] This parallels real-life social networks in that humans decide with whom to interact based on what questions or interests they may have at that time and as interests change so may their social network. Agents are heterogeneous in that they "can be diverse in their specifications and adopt 
different strategies to carry out interactions" [10], just as every human employs different strategies for interaction; for instance, a science student may want to have more experts very close by in their social networks, whereas an arts student may want more sociable people close by in their network. Concepts of expertise, interests and sociability are explored further in section 3.3.

\subsection{Oracle Agents and Concept Knowledge}

Agents in a referral network must be able to not only give answers and referrals but also disseminate concept knowledge in the form of suggestions, just as we do in real-life. The class of agents which contain concept relations are called oracles. Suggestions allow agents to learn about new concepts present in the network, especially ones related to their human operator's current area of interest.

There are various methods and architectures for capturing and utilizing concept knowledge. Current work tends to focus more on ontological solutions [11]; however, concept maps have been used as well [12]. Both these methodologies attempt to capture and use meta-data; i.e., data about data. Whether it is knowledge management or the Semantic Web, there is a need not only to share information, but also to share knowledge that gives information explicit meaning. This makes it easier for computer applications to automatically process and integrate information; for instance, knowing that a website is concerned with furniture tables rather than mathematical tables (matrices) would be useful to a web search application, especially if it knew a Math student was searching as opposed to a 
tradesperson, such as a carpenter. This thesis introduces oracle agents which are the primary source of concept knowledge; i.e., they are the holders of metaknowledge about the relationship of pieces of information within or across domains of knowledge.

\subsection{Trust}

An essential ingredient to any sort of social interaction is trust. The benefits of trust are many-fold and can vary from the reduction of risk to the reduction of complexity [13]. Every time we buy an airline ticket we are placing trust in the engineers and pilots of the airline that they have done their jobs correctly and the airplane will not crash unexpectedly; as George Carlin once put it "it's not flying l'm afraid of, it's the unexpected crash landing." The reason we can trust airlines is not that there have not been any unexpected crashes, but rather that there have been very few, making air travel relatively risk-free. However, it does not take too many unexpected events to change our minds. If the last few times we stepped on a plane it crashed, we would be rather hesitant to step on a plane again. In this way humans are initially risk-takers but once have been proverbially stung, tend to shy away. In this way trust acts as a mechanism to reduce risk.

Two major areas of trust establishment are: local and social. Local trust is created based on direct interactions with a trustee, whereas social trust is based on information from others on the trustee. Local interactions are considered more valuable in creating trust for two reasons, One, "since the trustor itself evaluates the 
interactions, the results are more reliable" [10] and two, "the context in which the trustworthiness of the provider is evaluated is explicit and relevant to the trustor." [10] For instance, in the example given above no matter how many people (social) told you it was safe to fly, if the last three time you (local) flew, the plane crashed, you would most likely still hesitate to fly.

Work by Singh and Yolum [1] has shown the emergence of trust based on prior direct and indirect (local and social) interactions and the ability of other agents to provide services across multiple domains and contexts. However, agents within a referral network must not only be able to trust answers and referrals they are receiving from other agents, but also they must maintain some measure of trust regarding the usefulness of concept knowledge they have received.

\subsection{Summary}

Peer-to-peer networks are an effective way to find distributed content. Built on the foundation of peer-to-peer networks referral networks provide a simple mechanism to find content based on social principles. The addition of oracles to referral networks allows a new type of referral, a suggestion, which allows agents to find new sources of related knowledge or expertise. As in any social setting trust is necessary not only in answers and referrals we receive, but in suggestions which prompt us to search for new sources of information. 


\section{Chapter 3}

\section{Current and Related Work}

\subsection{Introduction}

The concentration of this thesis is on collaborative social search; how communities can be utilized to both personalize and enhance search requests. This chapter presents a brief overview of related work as well as laying the foundation for the referral network architecture.

\subsection{Related Work}

When searching for information in either the online or real-world there are several important aspects:

1. An initial search query.

2. Sources of knowledge (oracles). The provision of knowledge on related concepts to reformulate a search query provides an important part of search (just as humans rephrase a search query when an existing one does not find desired results). Additionally, agents with concept knowledge can help distinguish context; for instance, the word matrix is a movie, mathematical construct, etc. 
3. Sources of expertise. Useful sources of expertise are the desired eventuality of any search. These so-called experts must be authoritative and trustworthy in order to be used.

This very general model is graphically depicted in Figure 2, where a person asking a question (1) utilizes knowledge from an oracle (2) to find a useful expert (3).

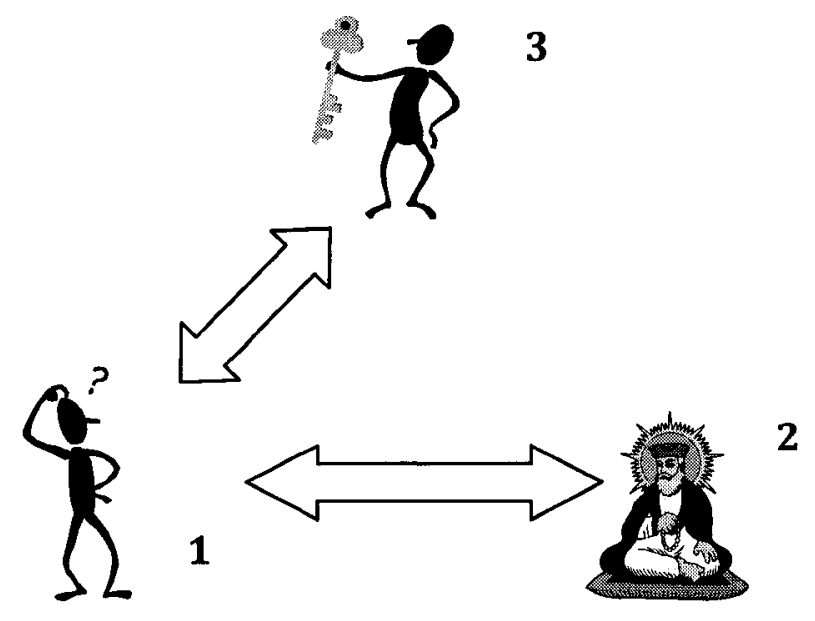

FIGURE 2: Agent - Oracle - Expert Interactions

The following sub-sections present several current solutions to better online searching, outlines work in referral networks and the emergence of trustworthy sources of expertise.

\subsubsection{Traditional Web Search}

Traditional search engines, such as Google, use web-crawling techniques to explore data on the web and then index content words in a data format that allows for fast 
access. [14] The frequency of search terms in a webpage is traditionally used to determine relevant pages. Furthermore, ranking is also done to web pages to determine relevance during search. A popular ranking technique is PageRank [15] which evaluates two things, "how many links there are to a web page from other pages, and the quality of the linking sites." [16] However, traditional search engines do not provide semantic constructs that allow the dissemination of related concepts to find new sources of knowledge or to denote context.

In addition to providing keyword-based search and authoritativeness ranking, most search engines apply some degree of classification to web pages. For instance, Yahoo.com ${ }^{3}$ has a directory of web pages which allows navigation by category instead of keyword. Yahoo also allows searching within a directory itself, allowing a limited amount of context to be given to a keyword. However, the categorization of knowledge is dependent on the professionals at Yahoo and is labour intensive and complicated to maintain and keep pace with the expansion of content on the web.

Abawajy and $\mathrm{Hu}$ in [17] present an application that utilizes the categorization capabilities of Google itself to classify search results returned to a user. Rankings are computed on a category-by-category basis and thus allow users to eliminate a complete set of results if a category does not fit their desired search criteria. This solution, however, is quite static and relies heavily on existing classification done by Google and does not provide a solution for finding related conceptual knowledge.

3 http://www.yahoo.com 


\subsubsection{Meta-Search Engines}

Meta-search engines provide a method to aggregate search results from several search engines. [18] An example of such a meta-search engine is InfraSearch pioneered by Gene Kan. [19] InfraSearch was built on top of the Gnutella protocol and exploited its ability to access and utilize heterogeneous sources of information. Search results were collected from any number of information providing nodes in the network. For instance, searching for the term "Nortel" may return results from financial nodes showing declining stock prices, while a photography node may return pictures of happier days on the Nortel campus. There is little information made available on InfraSearch; nevertheless, it is considered a motivating framework for work done in this thesis.

\subsubsection{Conceptual Map Searching}

Carvalho, Hewett and Canas, have used concept-maps to determine if the agents using knowledge represented in concept-maps improved the ranking provided by the search engines, and therefore "coming closer to the users' ranking of documents" [12]. Concept Maps first introduced by Novak [20] are "a twodimensional representation of a set of concepts that is constructed so that the interrelationships among them are evident." [12] Figure 3 shows a concept map modelling the notion of concept maps themselves. 


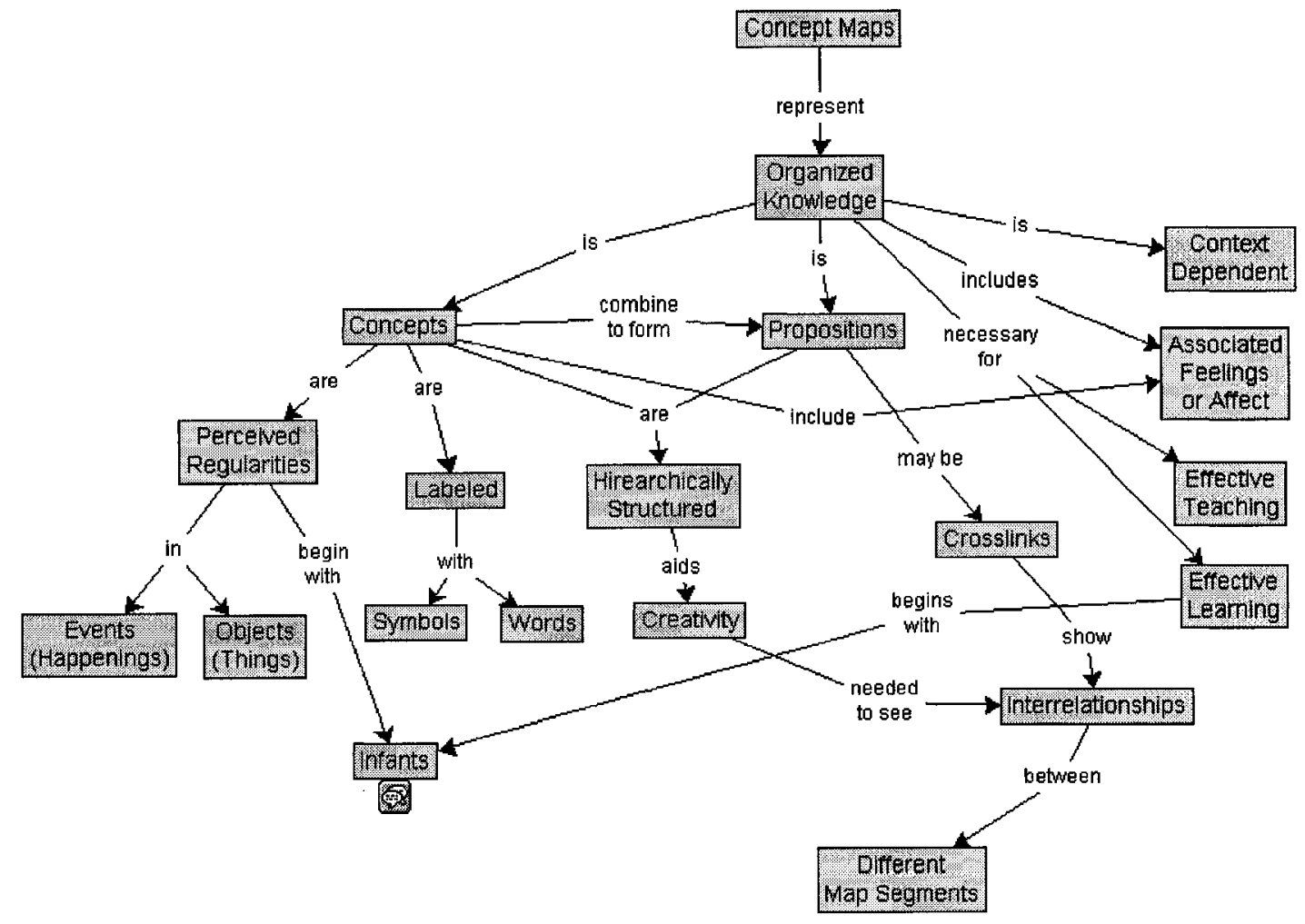

FIGURE 3: An example Concept Map

In work by Carvalho et. al. concept maps are utilized by agents to determine how many concepts are present in a document and their textual distance in relation to their existence and distance in the concept map. This work is domain specific and is meant to model how well a document (webpage) models a given domain given a set of concepts arranged in a concept map. Thus this research provides promise when searching for information that best represents a given concept, but does not provide the ability to distinguish between concepts when the ontology of discourse is not revealed. For instance, it could not provide useful information to a math student searching for information on "table" using a concept map for furniture tables. 
Additionally this work does not evaluate the information source on any sort of authoritativeness dimension.

\subsubsection{Ontologies and Recommender Systems}

Recommender systems attempt to alleviate the problem of information overload by learning preferences of a user and recommending new topics or sources of content in the same vein as the user's preferences. Supplementing recommender systems with ontological data has been shown to increase the accuracy of profiling a user and thus enables precise and useable recommendations to be given. [21] Ontologies are "a conceptualisation of a domain into a human-understandable, but machinereadable format consisting of entities, attributes, relationships, and axioms." [22] Referral networks can be utilized as recommender systems in that through a process of referrals agents can learn about new sources of content. It is the contribution of this thesis that through the addition of a simplified ontological construct (concept relations) the power of referral networks as recommender systems can be augmented.

\subsubsection{Collaborative Filtering}

Collaborative Filtering first introduced by [23] has been used to develop recommender systems [24] that attempt to aggregate and direct recommendations to similar people. A generalization of collaborative filtering has been presented in [25] which stipulates that collaborative filtering establishes the Implicit Culture phenomenon. Implicit Culture is defined as "the relation between two groups of 
agents such that the agents belonging to a group behave consistently with the 'culture' of the agents belonging to another" [25]. A System for Implicit Culture Support, Implicit, [26] [27] [28] provides a mechanism which is mainly intended to improve the web search of a community of people with similar interests. The goal of Implicit is to achieve Implicit Culture so "when a user submits a query, Implicit suggests specific information exploiting previous observations about the behaviour of other users when they asked similar queries." [26] This solution leverages the use of similar agents in a very efficient manner but still requires agents to first find a community of similar agents in order to leverage the utility of its implicit knowledge. Additionally the mechanisms by which other similar agents came to choose the information they have is not explicit and does not allow the agent to learn in any way from the recommendations of other agents. It is this problem that we address in this thesis.

Concepts used in collaborative filtering are also mirrored in "relevance" systems. A peer-to-peer relevance feedback application as described in [29] is used to provide the relevance of a search result based on users who made the same search in a peerto-peer network. For instance, a search for the image "sunset" would return results from peers throughout the network, but additionally annotated with the most common actions performed when searching for "sunset". Files can then be ranked in accordance to relevance based on download rate for example. This system, however, does not provide any contextual or semantic constructs for search queries. 


\subsubsection{Opinion-Based Filtering}

Work on recommender systems has expanded into the domain of decentralized agent systems with the advent of opinion-based filtering. Montaner et. al. introduce

"a technology that allows agents to look for similar agents that can offer them advice." [30] Once agents have found a group of friends with similar interests they can rely on these friends to provide recommendations. This technology successfully integrates elements of collaborative and content filtering as well as a measure of trust in neighbouring agents; similar agents giving unsuccessful advice will eventually be ignored. This thesis resonates with the core concepts of this work, (the importance of local evaluation, trust and a form of content filtering) however, it differs in one major respect. This thesis does not assume that similar agents will provide useful recommendations, answers or suggestions. Current referral networks provide a platform for the propagation of knowledge which will in turn aid agents in finding new and useful sources of expertise.

\subsubsection{Semantic Search}

The Semantic Web [31] provides a common standard (RDF, OWL etc.) for websites to publish the relevant information in a manner that more readily supports machine processing and integration. The use of the Semantic Web in search is introduced in a paper by [4] which utilizes the lightweight Alpiri Project (TAP) [32] semantic infrastructure to augment traditional searches. For instance, a search on Yo-Yo Ma may produce related results from a variety of websites on such things as current concert dates, ticket availability and to a biography of the artist. Guha et al. present 
the need for denotation of search terms and suggest the use of heuristics, such as specifying if you are searching for a person and what type of person to allow for more relevant search results.

The goal of the Semantic Web is quite different from the goal of this thesis and is only mentioned to contrast the two ideas. This thesis attempts to mirror the social process of search where suggestions are given to aid search queries, and the utility of these suggestions are subjectively rated by the agent. The Semantic Web, conversely, attempts to utilize objective ontological data to provide context for search. Although semantic constructs could provide useful concept relations, they are considered out of the scope of this thesis and no Semantic Web technology is used for search.

\subsubsection{Referral Networks}

Pioneering work on referral networks was done by Kautz et al. in ReferralWeb [33] which mined documents to uncover a social network of people. The ReferralWeb application modelled relationships statically, while work by Singh et al. introduced a framework that modelled "the relationship of agents dynamically by allowing them to adapt through neighbour changes." [34] This work allowed the study of "the emergence of authorities" [35] in a more dynamic type of network, and lead to the definition of a referral network as a "de-centralized agent-based approach for service location, where agents provide and consume services, and also co-operate with each other by giving referrals to other agents." [34] 
A major reason to believe that referral networks are useful for searching is that "referrals capture the manner in which people normally help each other find trustworthy authorities." [36] We, as humans, give referrals on a daily basis; however, indiscriminate referrals are seldom given. It is our ability to evaluate experts and to recall those assessments which provide the means for a rich and meaningful source of referrals. Referral networks parallel this real-life phenomenon by allowing agents to also evaluate past interactions with experts in the network and provide referrals based on those evaluations. Another powerful aspect of reallife social networks is their ability to form weak-ties [37]; loose connections across communities. It has long been known by sociologists that people with weak-ties are more useful than one with strong-ties (well-connected within a community), as they "add greater value by having different knowledge and perspectives." [38] Just about every person has a "social butterfly" friend who has at one point introduced them to someone helpful or interesting outside of their tightly-knit community of friends. In [39] the importance of pivot agents, which acted as weak-ties, was shown to improve the quality of the network, as agents had a means to connect across the network and were not constrained to isolated communities. This fact is useful in search as it allows better connectivity in the network and promotes discovery of hard-to-find information.

Service graph representations are also used in referral networks. Service graphs allow agents to capture relationships between service types; for example, "a service 
provider that has been found to be trustworthy for one type of service can be considered for another type of service based on how well the services relate." [40] A service graph represents a set of services connected by weighted edges denoting the likelihood that two connected services can be provided by the same service provider. A service graph is maintained by each agent and, based upon interactions with service providers, an agent determines its own set of weightings for the edges in the service graph. Agents with this model of services can then "promote" service providers for a new service "only if it has performed well for another service, and if performing well in the first service indicates that the provider may perform well in the second service." [40] Related service provision is learned from the experts themselves in the network, and though may be helpful in some cases, does not allow the reformulation of a query into a domain outside the area of expertise of the provider. It may be the case that a useful answer may lie in learning about a related concept that the provider cannot help the agent with. Additionally, the ability for contextual discrimination of concepts is not readily apparent in this framework.

In current referral networks, agents are allowed to dynamically move through the network but the interests of agents remain static. The ability for agents to adapt interests and search for new sources of expertise based on conceptually related information remains missing from current referral networks. We believe that these are important deficiencies; deficiencies that are the subject of research described in Chapter 6. 


\subsubsection{Trust in Referral Networks}

Since referral networks are meant to model "large distributed systems of autonomous and heterogeneous agents, it is generally inadvisable to assume that there are universally accepted trustworthy authorities that can announce the cooperativeness of different agents." [41] Thus an important research area in referral networks is the emergence of trust. Traditional search uses ranking algorithms such as PageRank [15] by Google to infer the authoritativeness of web pages and to offset search engine persuasion or web spamming [42]. However, a larger number of links to a page does not indicate authority as a malicious user may use "link farms" [43] to raise the rank of a particular web page.

Work by Yolum and Singh in [1] and [44] utilized service rating mechanisms to have trust emerge both locally and socially in referral networks. Thus agents now modelled the expertise and sociability (described in section 3.2.1) of other agents locally, allowing agents to verify the quality of not only answers but referrals in the network. Agents giving better answers or referrals were considered more authoritative or social, respectively, and were given higher ratings. Thus through local models of expertise and sociability, the property of trustworthy neighbours emerged in referral networks. This work revealed two important aspects of social search; firstly, the importance of local evaluation of referrals and answers and secondly, the utility of this local evaluation in finding authoritative experts. The work clearly reinforces the observation that trust is relative, not absolute and 
provides the motivation for the localized trust evaluation of concept knowledge presented later in this thesis.

\subsubsection{Summary}

Lacking in all of the aforementioned work is the ability of communities in social networks to leverage abstract relational concept knowledge to provide suggestions. Current collaborative filtering mechanisms allow recommendations based on existing preferences, but do not provide the discriminatory power of making suggestions. Conceptual mapping research allows a tighter fit between concepts and the information content of a document, but do not provide a mechanism to aid search or to redirect it usefully based on knowledge contained within a concept map. Current referral networks provide an efficient way to find information but lack the ability to find new sources of related information.

We now continue by describing a model of Referral Networks in more detail as it forms the basis of our extended model described in Chapter 6 .

\subsection{Current Referral Network Framework}

A referral network consists of 2 distinct entities: agents and their network of interconnections. The agents can be broken down into two sub-categories: consumers and providers. Consumer agents have a set of interests for which they are trying to find answers. The agents providing answers in the referral network are referred to as Providers and possess expertise in one or more domains. The goal of a 
referral network is to provide the mechanism by which a consumer agent can find a provider or expert (the words are used interchangeably within this thesis) whose expertise can best answer or best service the consumer's interests. The consumer agent attempts to find a provider (or an answer to a question if you will) by querying its neighbouring agents. The neighbouring agents may answer directly if they have enough expertise for the question at hand. However, they may not be experts in the domain that is being requested of them so they will not answer, but instead, in accordance with their referral policy, decide to give a referral to another agent who may be more knowledgeable in the given domain. A referral policy is a directive on how to refer an agent to another agent when an answer cannot be given directly [1]. Each agent maintains a model of its environment, which includes the expertise (the quality of service they provide) and the sociability (the quality of referrals they give) of agents who are neighbours (directly connected) or were neighbours at one point in time (may not be directly connected any longer). This model serves as a type of memory for the agent, as it holds current as well as previous models of interactions with other agents. After a certain amount of time asking questions, an agent may decide, based on its neighbour selection policy, to change its neighbours. For instance, I may want to have someone as my neighbour who is an expert in the area that I have been questioning or I may want to have a sociable neighbour who gives very good referrals to experts or providers in the network. Intuitively, a neighbour selection policy is a directive on who to keep as neighbours (or who to change to) and is weighted based upon whether sociable agents or expert agents are required as neighbours. 


\subsubsection{Modelling Interest, Expertise and Sociability}

Expertise, interest and sociability within a referral network are represented using the Vector Space Model [45]. Traditionally, the Vector Space Model (VSM) is used to index the content words of a document in an $\mathrm{m}$-dimensional vector, where $\mathrm{m}$ is the number of content words and the length of $\mathrm{m}^{\text {th }}$ component of the vector is associated with the term (word) frequency. This vector can then be compared against other vectors, thus enabling search functionality (Google employs this method for keyword searching). For knowledge representation in a domain, each term or dimension in an interest or expertise vector represents the amount of expertise or interest an agent has in that domain, normalized between 0.0 and 1.0 , where 1.0 represents a high interest or expertise in a domain and 0.0 represents a low interest or expertise in a domain.

In order for a referral network to function, the comparison of interest and expertise vectors is of great importance. There are three main evaluation metrics used. These are Similarity, Capability and PageRank. These metrics are described in the following 3 sections.

\subsubsection{Similarity}

To determine the similarity between two agents, a formula that is commutative is required. A common similarity measure is the cosine of the angle between two vectors, but this does not take effect of the length of the vector into consideration. 
Similarity is computed as shown in Equation 1 [46] below, where $I_{i}$ represents the interest vector of agent $i, I_{j}$ is the interest of another agent $j$ and $n$ is the dimension of $I_{i}$ and $I_{j}$ (they must be of the same length). Measured this way similarity is commutative, measures the Euclidean distance between two vectors and is normalized to obtain a result between 0.0 and 1.0 [1].

$$
I_{i} \otimes I_{j}=\frac{e^{-\left\|I_{i}-I_{j}\right\|^{2}}-e^{-n}}{1-e^{-n}}
$$

\section{EQUATION 1: Similarity}

\subsubsection{Capability}

A measurement of how good the expertise of an agent is for a given query is needed. This allows an agent to reply with a certain degree of surety or else give a referral. Capability resembles cosine similarity but also takes into account the magnitude of

the expertise vector [1]. This means that agents with more expertise will have a greater capability of answering the query. Capability is computed as follows [47]:

$$
Q_{i} \oplus E_{j}=\frac{\sum_{t=1}^{n}\left(q_{t} e_{t}\right)}{\sqrt{n \sum_{t=1}^{n} q_{t}^{2}}}
$$

EQUATION 2: Capability

In Equation 2, $Q_{i}$ is agent $i$ 's query vector $\left[\mathrm{q}_{1}, \mathrm{q}_{2}, \ldots, \mathrm{q}_{\mathrm{n}}\right]$ and $E_{j}$ is the expertise vector $\left[\mathrm{e}_{1}, \mathrm{e}_{2}, \ldots, \mathrm{e}_{\mathrm{n}}\right]$ of agent $j$, again $n$ is the dimension of $Q_{i}$ and $E_{j}$. 


\subsubsection{PageRank}

A ranking of agents within the referral network is used to measure the authoritativeness of agents and is computed using the PageRank [15] metric. This metric is used by Google to rank web pages returned from a query. In its classical setting of ranking web pages, PageRank is a measurement of how important a webpage is based on the links to it from other web pages on the Internet. In the setting of a Referral Network it is a measure of how authoritative an agent is and uses the number of other agents that have chosen the given agent as a neighbour. The PageRank of agents is computed as follows [1]:

$$
P(i)=d \sum_{j \in A_{i}} \frac{P(j)}{N_{j}}+(1-d)
$$

\section{EQUATION 3: PageRank}

In Equation $3, P(i)$ denotes the PageRank of agent $i, A_{i}$ is the set of agents that have $i$ as a neighbour, and $N_{j}$ is the number of agents that are neighbours of $j$. PageRank is a probability distribution used to represent the likelihood that a person randomly clicking on links will arrive at any particular page. The probability, at any step, that the person will continue surfing is a damping factor $d$ and is set to 0.85 as in the original paper. [48] 


\subsection{Summary}

There is an ever increasing need to supplement online searching with data that will enhance the search by either refining or generalizing it. The searching power of referral networks is combined with the simple notion of related conceptual information and localized trust to aid in searching across or within a domain. Having described related work and the current infrastructure of referral network, the next chapter describes algorithms for referral network evaluation and simulation parameters. 


\section{Chapter 4}

\section{Referral Network Simulation}

\subsection{Introduction}

The methodology for simulation used in this thesis is the same as that used in [1]. A network generation file is used to setup simulation policies, initialize vectors for interest, expertise and sociability, and assign random neighbours from a uniform distribution. A referral network simulation then runs for a number of cycles. During one cycle an agent can ask one or more questions based on their interests (query generation policy). The agent is then allowed to wait for all responses to and investigate any referrals (determined by a referral policy) given by other agents before asking their next question. Once all answers (as generated by the answering policy of responding agents] have been received the agent then updates its model of all its neighbours (using its learning policy). At the end of the cycle the agent is allowed to update its neighbours in accordance with a neighbour selection policy.

\subsection{Simulation Algorithms}

There are two main algorithms employed in a referral network. The first is the algorithm to ask queries and the second is to answer queries. Agents ask and evaluate queries in accordance with the following algorithm as defined in [1]. Lines 1 through 15 in the Algorithm 1 given below are repeated a set number of times (see 
simulation parameter in section 4.3 , ii) for every cycle. At the end of each cycle agents are allowed to change neighbours; the number of cycles is determined by the simulation parameter described in section 4.3 , i.

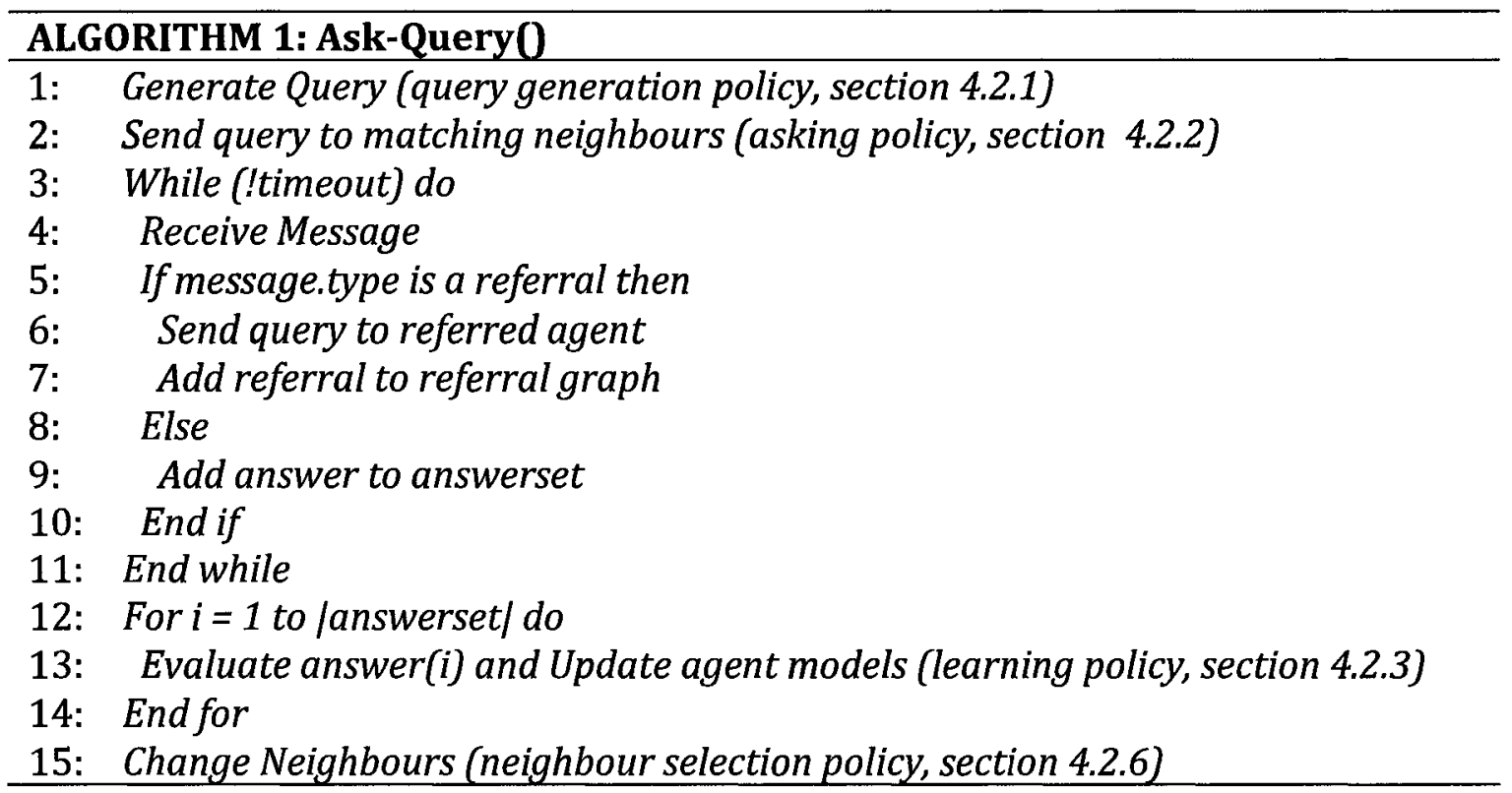

\subsubsection{Query Generation Policy}

Since there is no user interaction during a simulation, a policy for generating queries is required. The query generation policy fulfills this need by simply iterating over each index in the interest vector of the agent and perturbing the value by some small random amount from a uniform distribution. This newly perturbed interest vector is then used as the query.

\subsubsection{Asking Policy}

The asking policy is responsible for determining if the neighbour agent should be asked the given query. The default implementation of this policy uses the current 
agent model's of all neighbours and determines if the neighbour is capable (using the capability metric) of answering for the current query. If the capability metric is above a pre-defined threshold (section 4.3 , xi) then the neighbour is queried.

\subsubsection{Learning Policy}

The first responsibility of the learning policy is to evaluate the answers received for a query. If the similarity of current query and an answer (as determined using the capability metric) is above a given threshold (section 4.3, viii) then the answer is graded as good otherwise it is graded bad. The best answer given to agent $i$ is chosen using Equation 4.

$$
\forall r \in R ; B_{i}=\max \left(r \oplus Q_{i}\right)
$$

\section{EQUATION 4: Best Answer}

Where $R$ is the set of all responses (answers) from agents who responded to the query $Q_{i}$ from agent $i$, and $\mathrm{B}_{\mathrm{i}}$ is the best answer given to agent $i$.

The learning policy then updates three major areas, the modelled expertise and sociability of the agents which have provided answers or referrals and the expertise of the agent asking the question. The modelled sociability vector of agent $j$ is updated according to Algorithm 2 if it gives a referral that leads to a good answer. 


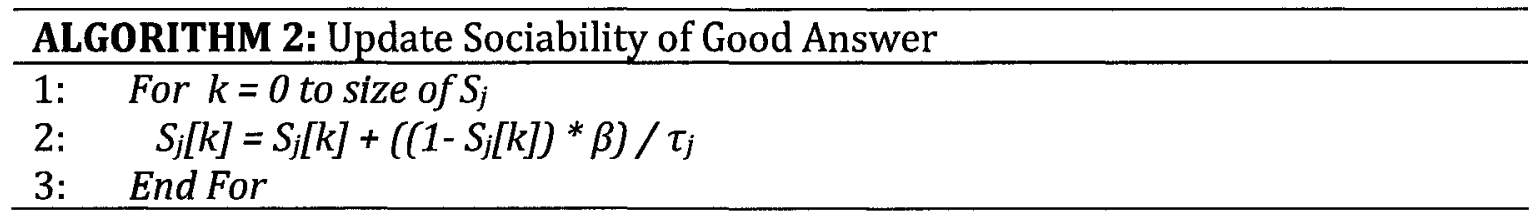

And if a bad or no answer is given the sociability of agent $j$ is updated using Algorithm 3.

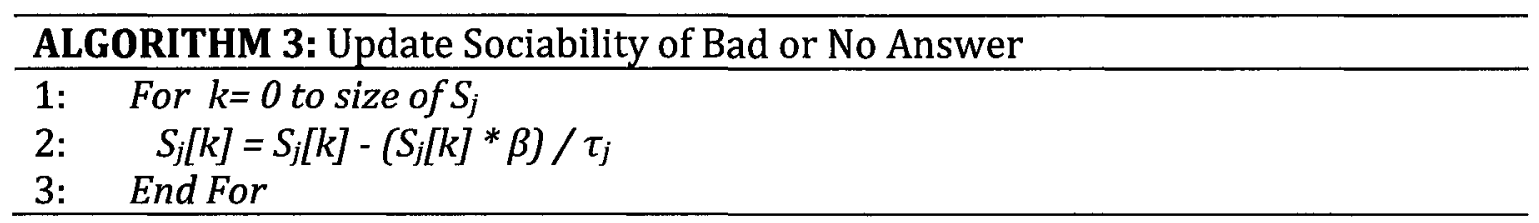

In both algorithms, $\beta$ denotes a weighting coefficient (see section 4.3 , vi), $\tau_{j}$ denotes the distance (as recorded in the referral graph for the query, line 7, Algorithm 1) between agent $j$ and the agent who provide agent $i$ a good, bad or no answer at all, and $S_{j}$ represents the modelled sociability vector of agent $j$. The referral graph is used to ascertain $\tau_{j}$ and is a directed a-cyclical graph (DAG) rooted at agent $i$ (which made the query), contains a finite set of agents (vertices) and a set of referrals (edges). The properties of the DAG are explained in [41] and are left out of this thesis for the sake of conciseness.

After updating the sociability of an answering agent, the modelled expertise of answering agents as well as the expertise of the querying agent is updated. The expertise of the agent $i$ making the query updates the modeled expertise of agent $j$ who gave an answer, using Algorithm 4. This algorithm is used for all agents that give an answer. 


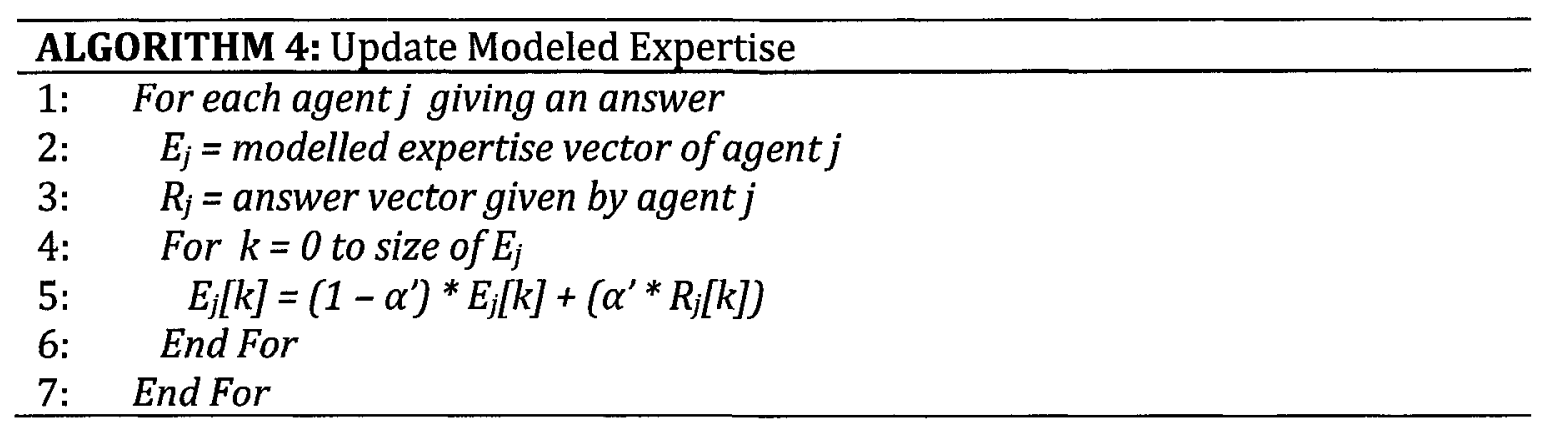

$E_{j}$ denotes the modelled expertise vector of agent $j$ giving the answer, and $\alpha^{\prime}$ (section $4.3, \mathrm{v}$ ) denotes a learning coefficient.

To update its own expertise $\left(E_{i}\right)$ the querying agent uses the best answer given $\left(B_{i}\right)$ and a different learning coefficient $\alpha$ (section 4.3, iv). Algorithm 5 formalises the computation.

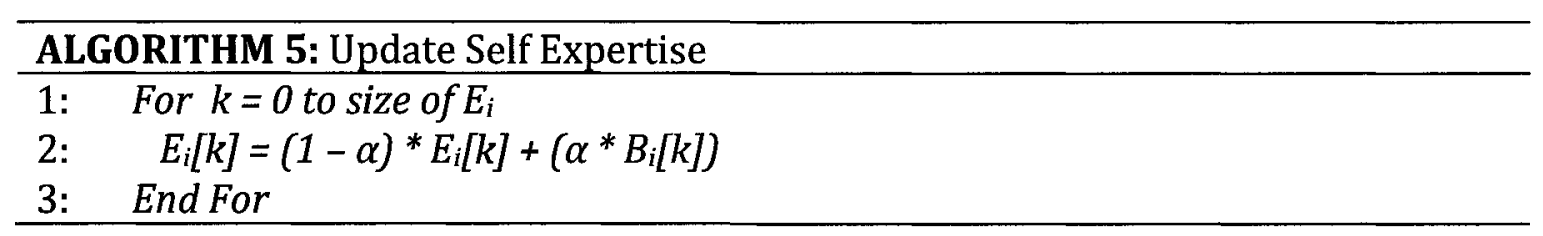

During each cycle an agent will invariably receive queries which it will attempt to answer by using Algorithm 6 from [1]. In Algorithm 6, the agent receiving the query is $j$ and the agent posing the query is $i, E_{j}$ denotes the expertise of agent $j$, and $Q_{i}$ denotes the query posed by agent $i$. 


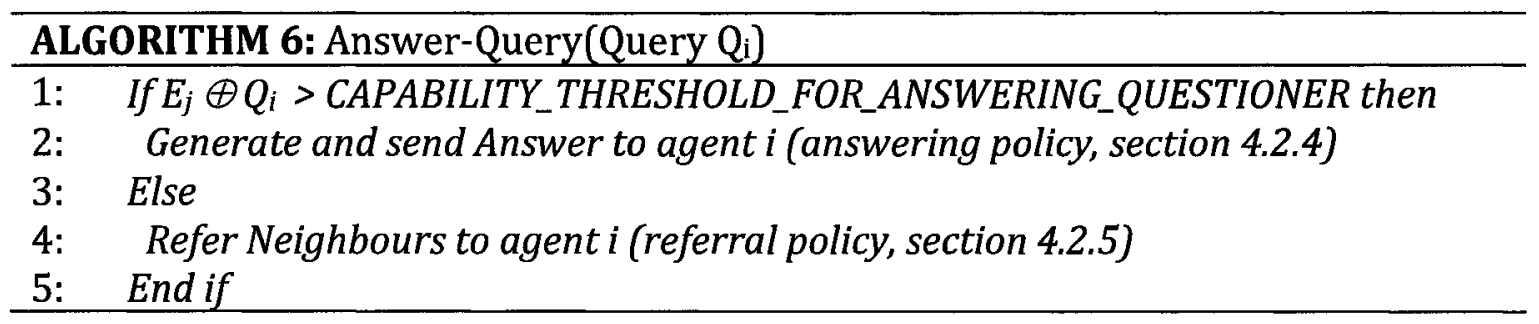

\subsubsection{Answering Policy}

The capability metric (Equation 2) is used in line 1 of Algorithm 6 to determine if the agent has sufficient expertise to answer the query. The answer is then generated by perturbing the expertise vector by either some small amount or not at all (the latter was done in this thesis).

\subsubsection{Referral Policy}

The capability metric is also used when determining which neighbours to refer (line 4). The query is compared to the modelled expertise of the neighbouring agents and if sufficiently similar the agent is referred. There are three main referral policies Refer All, Refer All Matching, and Refer Best. The Refer All policy refers all neighbours to a querying agent, regardless of the neighbour's ability to answer the current query. Refer All Matching only refers neighbours whose modelled expertise capability exceeds some threshold (section $4.3, \mathrm{x}$ ). Refer Best will only refer the neighbour best suited to answer the query; i.e., the best expertise.

\subsubsection{Neighbour Selection Policy}

Once an agent has completed asking and answering a set of questions, they are allowed to choose new neighbours based on their updated agent models (as 
determined in line 14 of Algorithm 1). An agent must always keep a static number of neighbours; thus if an agent decides to choose a new neighbour it must drop an existing one. The neighbour selection policy utilizes Algorithm 7 to change neighbours.

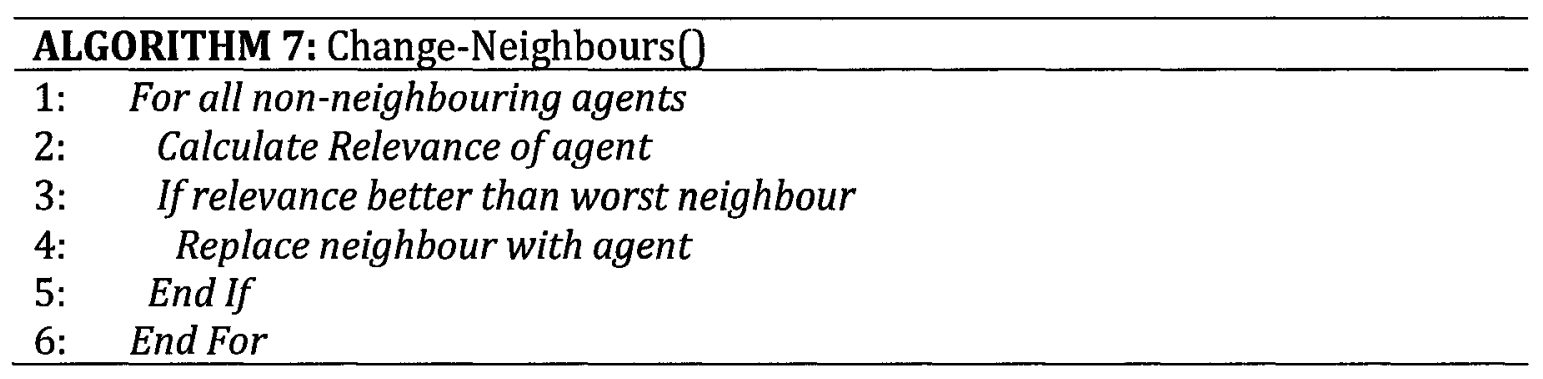

Let $i$ denote the agent changing neighbours and $j$ one of its non-neighbours. The relevance of agent $j$ (line 2) to agent $i$ is calculated according to Equation 5 .

$$
\operatorname{Rel}_{i}(j)=(1-W)\left(I_{i} \oplus E_{j}\right)+W\left(I_{i} \oplus S_{j}\right)
$$

\section{EQUATION 5: Relevance}

Where $W$ (section 4.3 , vii) is a coefficient weighting the importance of expertise or sociability in the calculation, $I_{i}$ indicates the interest of agent $i, S_{j}$ and $E_{j}$ denote the modelled sociability and expertise respectively of the non-neighbouring agent $j$. As described in Chapter $3, \oplus$ denotes the capability metric. 


\subsection{Simulation Parameters}

There are several parameters that are essential to the functioning of a referral network simulation they are described below.

i. NUM_NEIGHBOR_SET_CHANGES: This parameter denotes the number of cycles in the simulation.

ii. NUM_QUESTIONS_PER_NEIGHBOR_SET: The number of questions the agents are allowed to ask each cycle. A higher number for this parameter will result in more queries to neighbours and thus allow the agent to better model the expertise and sociability of a given neighbour.

iii. MAXIMUM_PERCENTAGE_PERTURBATION_FOR_GENERATING_QUERY:

The maximum amount the interest vector is allowed to be perturbed at any given index when generating the query.

iv. $\alpha$ : A learning coefficient used when calculating the amount to increase the expertise of an agent which received a good answer. As this value increases more emphasis is placed on the received answer than the current expertise of the agent.

v. $\boldsymbol{\alpha}^{\prime}$ : Another learning coefficient utilized when calculating the amount to update the modelled expertise of another agent which gave the questioning agent an answer (good or bad). The default value for this simulation parameter is 0.25 ; using this value allows agents to slowly build up their models, giving them a measure of stability in spite of variability in answers received. 
vi. $\quad$ : A coefficient used when calculating the amount to update the modelled sociability of another agent which gave the questioning agent an answer (good or bad). The default value for this simulation parameter is 0.2 ; this allows both good and bad referrals to be penalized fairly moderately, allowing agents a measure of stability in the face of variability in quality of referrals.

vii. W: denotes the weight of sociability in choosing neighbours. When set to 0 only the expertise of the agent is utilized (denoted as the Experts neighbour selection policy), when set to 1 only the sociability of the agent is used (denoted as using a Sociable neighbour selection policy). A value of 0.5 would indicate a weighted average.

viii. CAPABILITY_THRESHOLD_FOR_EVALUATING_ANSWER: The threshold used by the Learning Policy to determine if an answer is good or bad. The capability metric is applied to the query and answer provided and if above this threshold is evaluated as a good answer.

ix. CAPABILITY_THRESHOLD_FOR_ANSWERING_QUESTIONER:

This threshold is used by the answering policy to determine if the agent's expertise is sufficient to answer the given query. If the capability metric returns a value less than this threshold the agent will attempt to send a referral. Intuitively this threshold should be set to something higher than the threshold for evaluating an answer, so as to ensure that the answer given will be a good one. 


\section{CAPABILITY_THRESHOLD_FOR_REFERING_QUESTIONER_TO_NEIGHBOR:}

This threshold is used by the referral policy to determine if the agent should refer a neighbour based on the neighbours modelled expertise and the current query. This threshold is usually set to a value higher than the threshold for answering, so as to ensure that an agent will more often than not refer an agent which can answer the query.

xi. CAPABILITY_THRESHOLD_FOR_ASKING_NEIGHBOR: The asking policy uses this threshold to determine if the agent should ask its neighbours. This threshold is usually set very low to ensure that a majority of an agent's neighbours are contacted.

\subsection{Summary}

The lifecycle of an agent involves utilizing a number of policies to send and receive queries, and generate referrals and answers. These policies give agents their heterogeneous and autonomous properties. A group of simulation parameters guide several of the policies in making decisions such as neighbours to send queries to and which neighbours to refer. Once a referral network simulation completes, performance metrics are computed and results obtained as to the effectiveness, efficiency and authority of the referral network and its associated setup. These metrics and the results of Yolum and Singh are presented and explained in the subsequent chapter. 


\section{Chapter 5}

\section{Measuring Performance of Referral Networks}

\subsection{Introduction}

Once a simulation has ended several metrics can be used to evaluate the policies, simulation configuration parameters and structure of a referral network. There are three main evaluation measures for referral networks. They are effectiveness, efficiency, and authoritativeness. These metrics are described in the next 3 subsections. Additionally, factors affecting network structure are examined.

\subsection{Effectiveness}

The effectiveness of a system measures how easily agents find useful providers [1]. There are two metrics used to measure effectiveness. The first is direct quality, which is measured as the usefulness of the direct neighbours of an agent, in terms of their expertise and the agent's interests. The second is $n^{\text {th }}$ best quality. This metric takes into consideration "how well the agent's interest matches the expertise of all other agents in the system, scaled down with the number of agents it has to pass to get to the agent" [1]. So agents further away in the social network will contribute less to the quality of a given agent. The value of $n$ is taken to be twice the number of neighbours for a given agent as in [1]. The reason $n$ is chosen in this manner is to 
ensure that it measures how close agents are getting to experts in the system. The contribution of agent $j$ to agent $i$ 's quality is given by:

$$
\frac{I_{i} \oplus E_{j}}{\operatorname{path}(i, j)}
$$

\section{EQUATION 6: Quality Contribution}

In Equation 6, $I_{i}$ refers to the interest vector of agent $i$ and $E_{j}$ denotes the expertise of agent $j$. The function $p a t h(i, j)$ is the distance between the $i^{\text {th }}$ and $j^{\text {th }}$ agents and is computed using the Bellman-Ford algorithm [49]. The $n^{\text {th }}$ best quality metric is computed by calculating all qualities (using Equation 6) from an agent to all other agents in the network, and then taking the $n^{\text {th }}$ best measure from a sorted list. In this way the quality is more reflective of how close (but not directly connected) an agent is to experts in the network.

\subsubsection{Effectiveness Results}

It has been shown in [1] that when increasing the threshold to refer a neighbour; i.e., moving from the "refer all" policy to the "refer all matching" policy that the averaged value of the $n^{\text {th }}$ best quality metric increases. Yolum and Singh make the following observation, "among these referral policies Refer All Matching results in graphs with higher quality, where the best threshold increases with the percentage of experts in the society." Yolum describes this in [10] as follows, "Exchanging more referrals does not guarantee that the quality of the network will be high. The structure of the network can prevent consumers from locating some of the service providers". For 
instance, a high number of referrals do nothing in the way of aiding the querying agent in finding a good answer if all of an agent's neighbours cannot give good referrals. The results are graphically illustrated below in Figure 4, from [10].

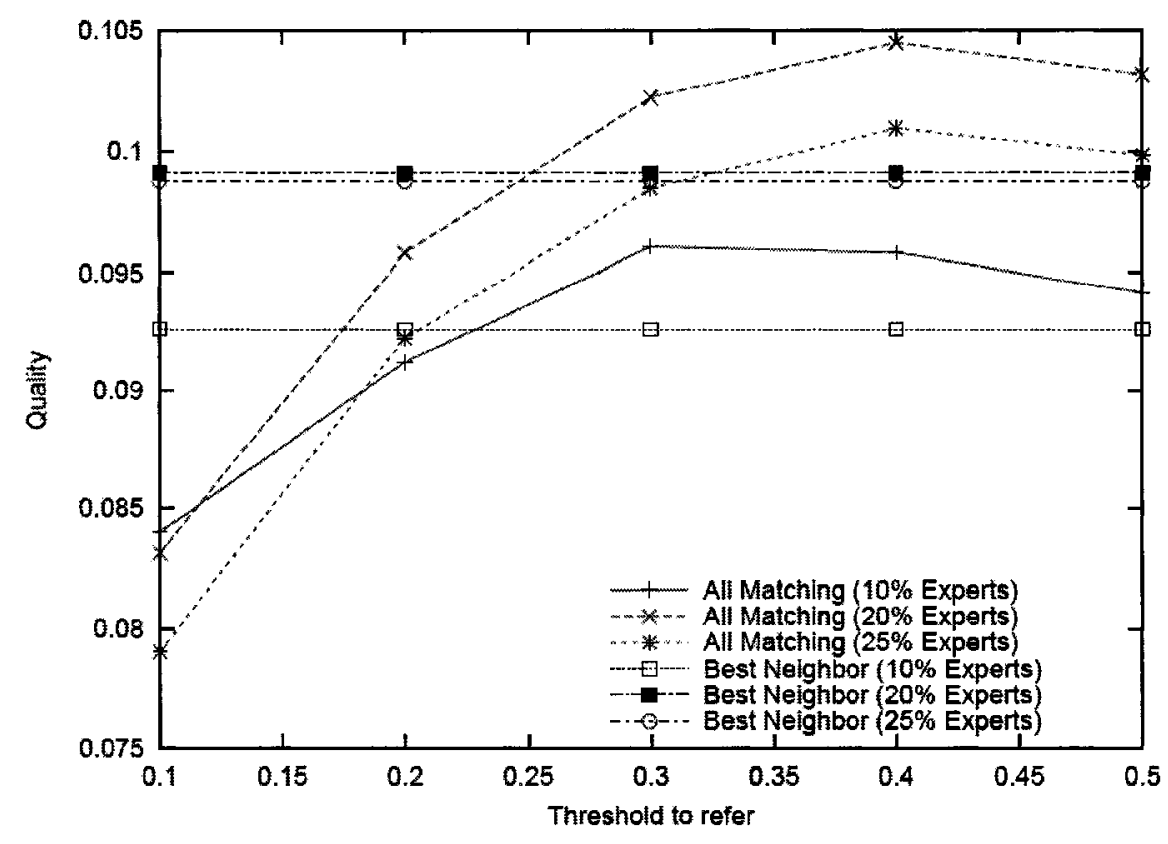

FIGURE 4: Effectiveness

There are some important observations to make about these results:

1. The average distance between agents decreases as the threshold to refer increases. With fewer referrals there was less convergence on experts in the system and thus shorter paths were found through the network to any given agent.

2. The lower the threshold to refer, the more agents converged on fewer experts in the system. 
3. Experts do not necessarily have interest in the same domain as their expertise, thus they most often did not have similar experts as neighbours.

Using these observations Yolum's results were replicated by tuning the simulation in one of the following ways:

1. Experts did not have high interest in the areas of their expertise and thus experts in the same domain did not seek each other out. Communities were formed around experts instead of around a group of experts.

2. Experts were spread across many domains and allowed to have interest in the same domain as their expertise.

CONCLUSION. Without connectivity between experts in a referral network, the threshold to refer (section $4.3, \mathrm{x}$ ) acts to increase quality as it mitigates convergence on fewer experts. Convergence serves to increase direct quality (the agent has experts as neighbours) but tends to isolate consumer agents to a set of experts. This causes either no path or only a long path to exist between the consumer agent and it $\mathrm{n}^{\text {th }}$ best neighbour, and thus leads to a worse quality measure. More selective referrals leads to a better quality measure as it results in less convergence and thus a shorter path to more experts in the system.

\subsection{Efficiency}

The quality measure of a network graph is optimistic [1] since it is possible that a provider may not respond and other agents may not make useful referrals. Thus, a 
high quality graph does not mean that an agent will reach the service it is trying to find. A new metric is introduced to measure the efficiency of finding answers. Efficiency is defined as the ratio of correct answers received to the number of agents contacted [1]. Efficiency is a measure of how well experts are responding to questions within the network. Accuracy is measured over all agents in the network and is calculated using Equation 7. The number of correct answers is the sum of correct answers received by all agents; the total questions asked is the sum of all questions asked by agents.

$$
\text { Accuracy }=\frac{\text { Correct Answers }}{\text { Total Questions asked }}
$$

\section{EQUATION 7: Accuracy Equation}

\subsubsection{Efficiency Results}

Similar to the effectiveness metric, the efficiency metric is dependent on the setting of the threshold to refer simulation parameter (section 4.3, $\mathrm{x}$ ). When this threshold is set low, the referrals become less selective and more agents are contacted, thus lowering the efficiency. If it is set too high, then referrals are too restricted and not enough agents are contacted to find useful answers. These results are shown graphically in Figure 5 below from [10]. 


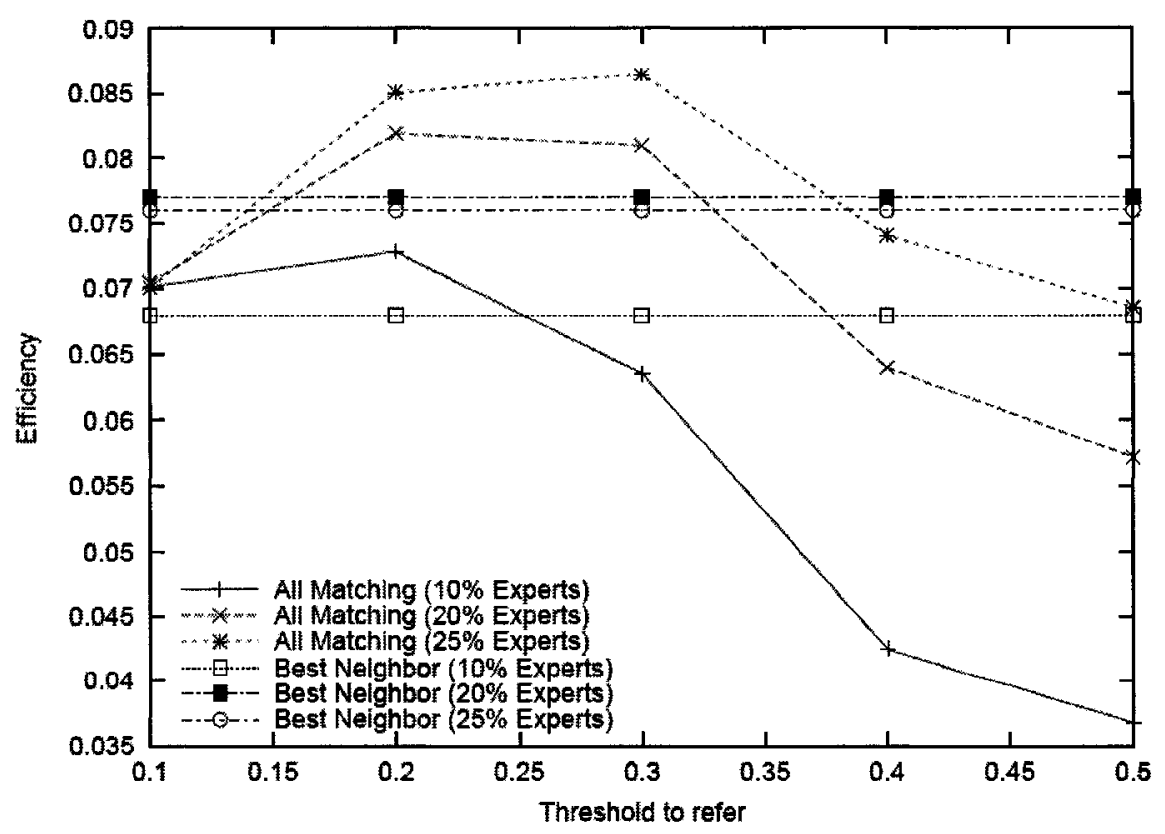

FIGURE 5: Efficiency

CONCLUSION. Both low and high thresholds to refer suffer from reduced efficiency as a low threshold contacts too many agents in proportion to good answers and a high threshold does not allow enough agents to be contacted to supply good answers.

\subsection{Authoritativeness}

Some agents will be chosen as a neighbour by a greater number of other agents, and are thus identified as more authoritative. They may be more sociable or have a high expertise (and better able to answer queries within their domain of knowledge), and thus are chosen as a neighbour more often than other agents who are less sociable or have a smaller expertise value. The authoritativeness measure is 
computed using the PageRank metric (section 3.3.4) to study the emergence of authorities in referral networks.

\subsubsection{Authoritativeness Results}

Yolum and Singh have found that more authorities emerge through the Refer All Matching Policy, but that the Refer All Policy causes the emergence of authorities whose level of authoritativeness is higher. The results are shown in Figure 6 from $[10]$.

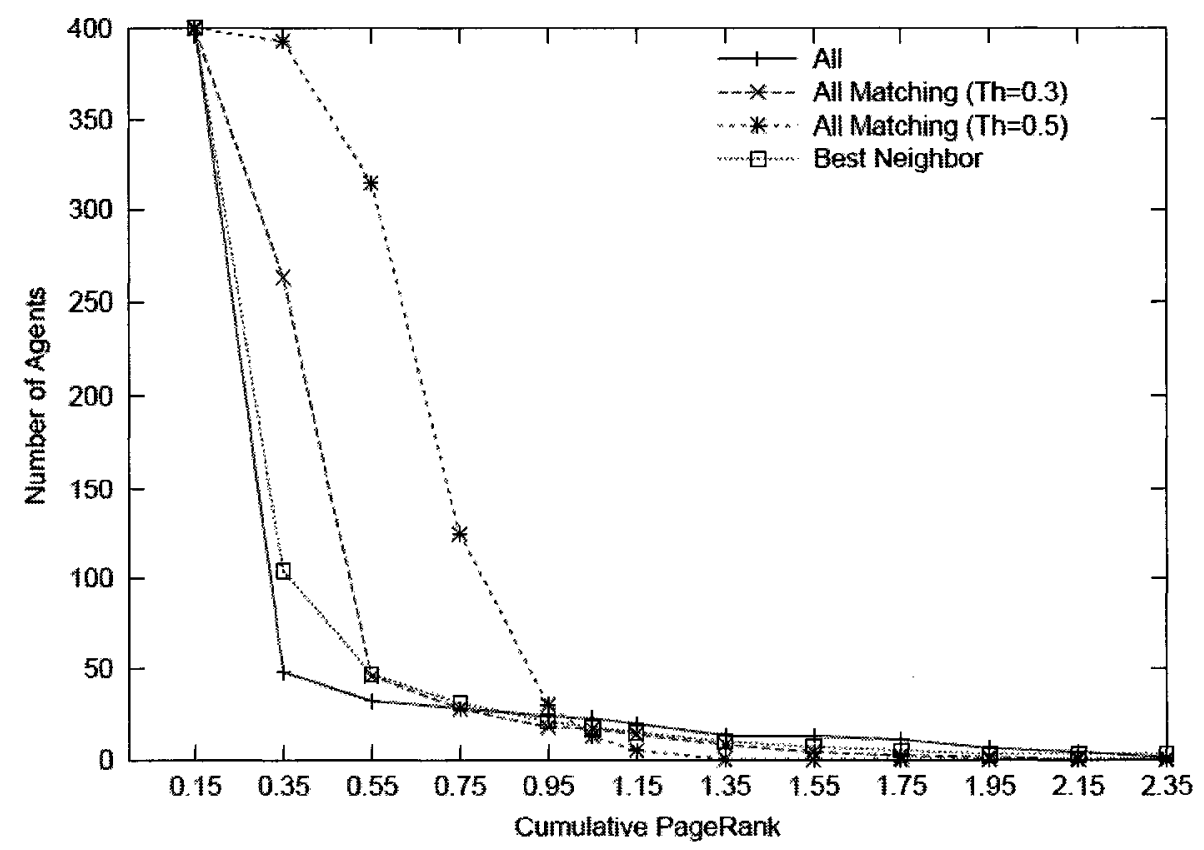

FIGURE 6: Authoritativeness

CONCLUSION. As the threshold to refer is increased authority is more distributed across agents as less referrals to experts in the network are given. Lower thresholds 
cause a larger amount of convergence and thus lead to more agents having a higher PageRank.

\subsection{Network Structure}

The neighbour selection policy profoundly affects the structure of the network. Recall that each agent chooses its neighbours based on local information, and is not managed by any centralized means. As agents change neighbours it may be to the advantage of the agent, but the resulting graph may be undesirable. Work by Yolum [1] has shown that if there are more providers than the number of neighbours an agent can have, agents looking for only providers in the network will cause the network to degenerate to a bi-partite graph. The quality of a bi-partite graph is stable and suboptimal as consumer agents have solely chosen providers and thus can make no referrals to other consumer agents. [1] Conversely if agents attempt to keep only sociable agents (that give referrals) as neighbours the graph will contain more than one weakly connected component. A weakly connected component of a graph is a maximal sub-graph that would be connected when the edges are treated as undirected. Thus there will be consumers that cannot reach one or more service providers as they have chosen only sociable agents as neighbours. A safe policy to use for neighbour selection is a weighted average that allows an agent to have both provider and sociable agents as neighbours.

Interest clustering is also an important aspect of a referral network structure and is defined as a "coefficient to measure if similar agents become neighbours." [1] 
Interest clustering measures how similar the interest vectors of an agent i's neighbours (including I itself) are to each other. [1] Equation 8 is used to compute interest clustering. The set containing agent $i$ and all its neighbours is denoted by $N_{i}$ and $E_{i}$ denotes all edges between the nodes in $N_{i}$.

$$
\gamma(i)=\frac{\sum_{(i, j) \epsilon E_{i}} I_{i} \otimes I_{j}}{\left|N_{i}\right|\left(\left|N_{i}\right|-1\right)}
$$

\section{EQUATION 8: Interest Clustering}

Yolum et al. make the observation that when agents use the Provider policy for neighbour selection $(\mathrm{W}=0$, section 4.3 , vii), interest clustering tends to decrease, while when using the Sociables policy $(\mathrm{W}>0.5)$ interest clustering increases. Agents with similar interests will tend to give better referrals and thus if an agent prefers sociable neighbours will most likely chose one with similar interests. This finding by Yolum necessitates that consumer agents must not have the same interests as experts who provide useful answers.

\subsection{Summary}

A rich set of experimental results exists for referral networks. The results show that being more selective with referrals can lead to less convergence and therefore increase the global quality of a network. However, being too selective reduces the number of referrals and may cause experts not to be found in the network, leading to agents not getting good answers. The distribution of authority as measured by 
PageRank shows how referral thresholds also affect the emergence of authority. Simulation parameters affecting neighbour selection will ultimately determine the referral network structure. Still, referral networks explained thus far lack the ability to adapt their interests and a mechanism to model some sort of semantic construct to aid in the adaptation of interests. This is explored next, in Chapter 6. 


\section{Chapter 6}

\section{Concept Relations in Referral Networks}

\subsection{Introduction}

This chapter introduces three fundamental aspects missing in state-of-the-art referral networks; firstly, the relation of concepts, secondly the propagation of this knowledge throughout the network and thirdly, the ability of an agent to dynamically change their interests based on newly acquired concept knowledge.

\subsection{Real World Concept Relations}

In the real world, humans innately relate concepts; for example, dog is to animal what apple is to fruit. It is our ability to relate concepts and form hierarchies of relations that allows us to effectively process and generalize information in our dynamic world. This concept relation knowledge is of great importance; it allows us to change our interests to reflect new found knowledge, rephrase questions in a manner that can return better search results, and give suggestions to people asking us questions, in the form, "Did you mean this instead of that?". For instance, within a real-life social network, if you ask a mathematician about Einstein's Theory of Relativity the mathematician may not be able to answer you with same level of expertise as a physicist because he has little knowledge of the theory, but he may have enough relational concept knowledge to give you a suggestion on what you 
should ask for instead. He may tell you, "Instead of asking me about Einstein's Theory of Relativity, why don't you ask me about Einstein's field equations?"; both are topics in the same domain and tightly related to each other. The mathematician is most likely very knowledgeable about the intricacies of the mathematics involved in Einstein's Field Equations and would give good answers to questions in that domain. Provided you could understand the mathematics involved in Einstein's Field Equations, learning about them would no doubt help augment your knowledge of the Theory of Relativity. Thus learning that a topic is related to another and then learning about this new topic, in the long run educates you on the original topic. It is this hypothesis that motivates the proposal of the additional components to referral networks described in this chapter.

Within the Vector Space Model (VSM, section 3.3.1), which is used to model expertise and interest in a referral network, it is quite possible that the expertise vector of an expert and the query vector of a consumer are very different upon analysis using the similarity metric described in Equation 1. However, it is quite plausible that the interests of the consumer lie in the same domain as the expertise vector of the expert and may even be tightly related. Thus a small amount of concept relational knowledge would be of great benefit, if the consumer were aware of it. This is the essence of the algorithms provided by this thesis and the primary motivation for the addition of a new type of agent - the oracle. 
As can be seen visually in Figures 7, 8, and 9, being aware of relational concept knowledge allows an agent to dynamically change its interests to better learn about concepts in the domain of its interests. Figure 7 depicts the expertise vector of an expert in the domain of Einstein's Field Equations, while Figure 8 depicts a consumer interested in the Theory of Relativity. If a consumer were to ask the expert in Figure 7 about Einstein's Theory of Relativity it would not get an answer (or at least not a very good one). However, after learning that Einstein's Field equations and the Theory of Relativity are related, if the consumer were to modify its interests placing more of an onus on Einstein's Field Equations and ask a question based on these new found interests, the expert would then be able to answer with a much better degree of accuracy. This would lead to an improved, presumably satisfactory, response and thus the querying agent would learn about Einstein's Field Equations and thus the Theory of Relativity.

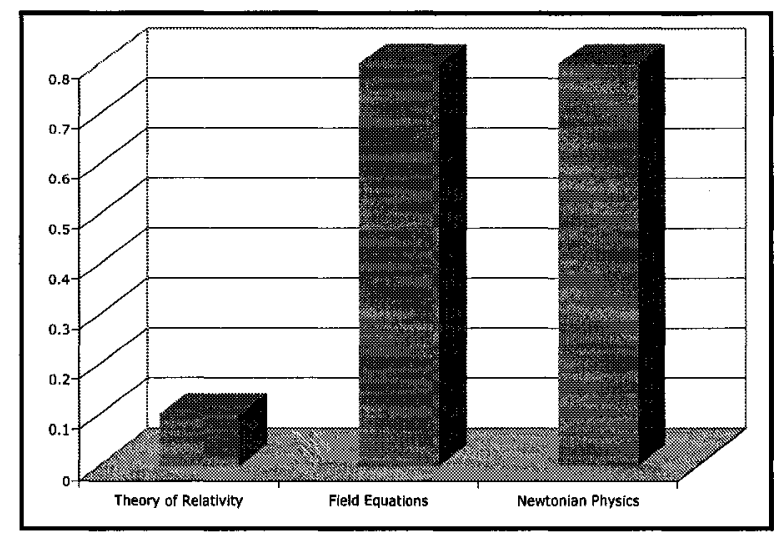

FIGURE 7: Example Expertise Vector 


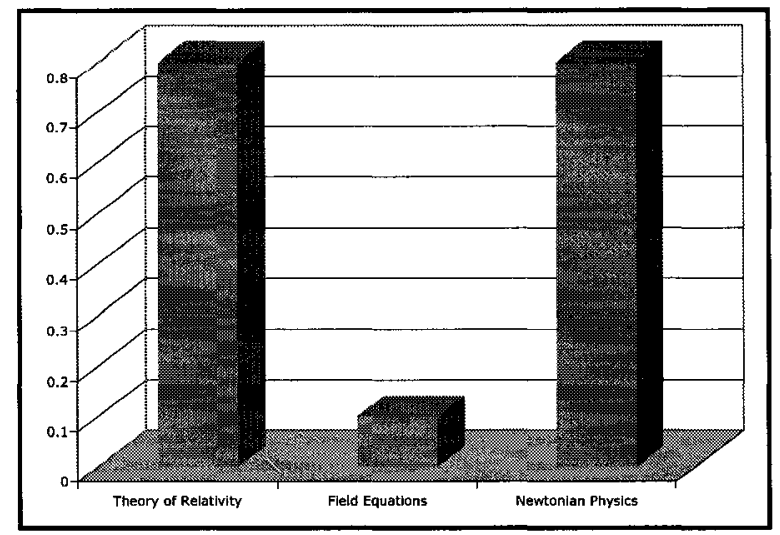

FIGURE 8: Consumer Interest Vector Before

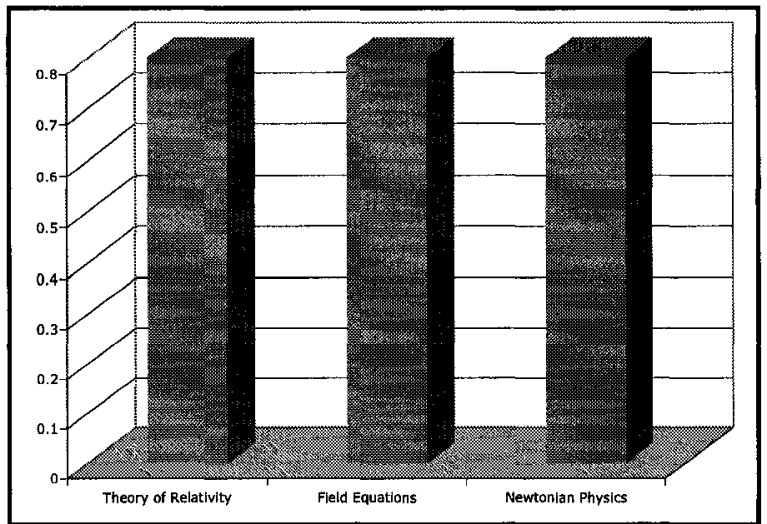

FIGURE 9: Consumer Interest Vector After

Notice that interest vector in Figure 9 now matches the expertise vector in Figure 7 in two of the three areas instead of one of the three areas as in Figure 8. This will result in the query (based on the interests of the querying agent) being more similar to the expertise vector of the expert and thus is more likely to overcome the capability threshold and be answered by the expert.

For a referral network to function as described above, concept relational knowledge needs to be introduced into the model along with policies for its usage. These are described in the next 2 sub-sections. 


\subsection{Suggestion and Interest Adaptation Policy}

For a referral network to function as described above, two new policies need to be introduced. One is a suggestion policy and deals with what concept knowledge an agent will suggest to another agent upon receiving a query. The second is an interest adaptation policy and functions in the capacity of updating the interests of an agent that has concept knowledge about related topics.

\subsubsection{Suggestion Policy}

Within the referral network simulator the suggestion policy is used when an agent is asked a question. Algorithm 6 introduced in section 4.2 is modified in the following manner. The text in bold indicates the changes that are required.

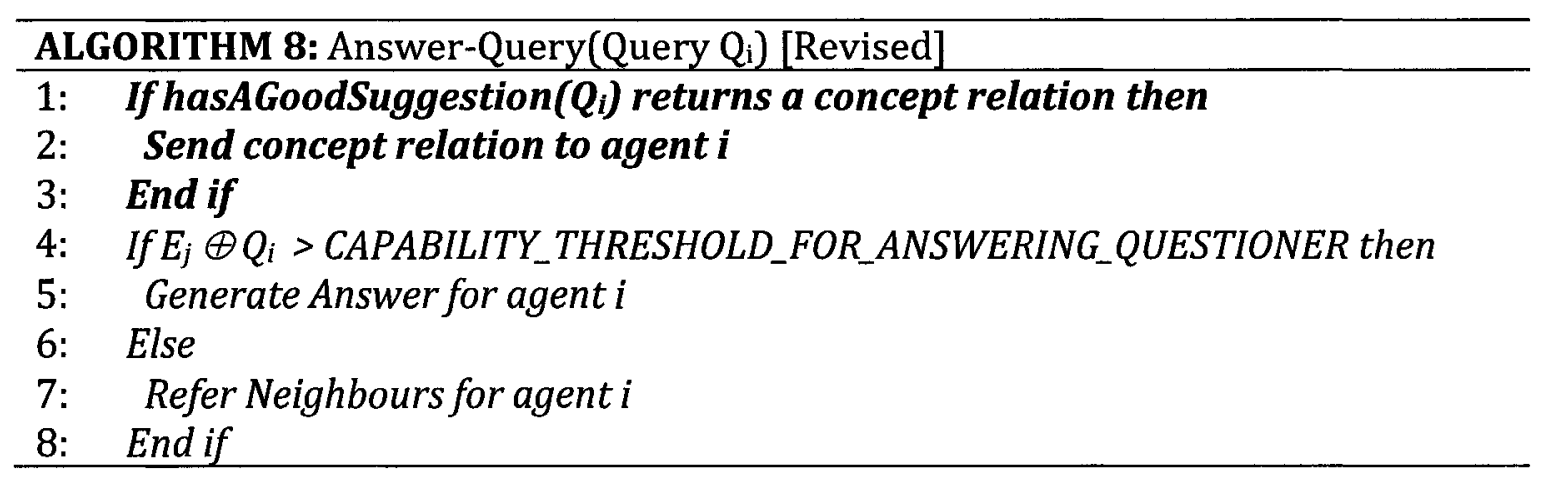

The oracle agent with concept knowledge now contains a set of concept relations (an agent could contain more than one concept relation) described in Data Structure 1. Concept relations are modelled very simplistically in the simulation, by containing two numbers representing the indexes within the VSM that are related. The 
concepts are unidirectional, that is domainA is related to domainB; the inverse relation is not implied. In this way agents always know which domain they originally had interest in (domainA) and which domain (domainB) to search in. Numbers are used to represent domains throughout this thesis; thus a concept relation relating domain 0 to domain 1 is denoted as $0 \rightarrow 1$.

\section{Data Structure 1: Concept-Relation}

1: int domainA;

2: int domainB;

3: Agent suggestingAgent;

To determine a good suggestion for the given query the agent must determine the best concept relation in accordance with Algorithm 9. The policy outlined below is a stateful suggestion policy that attempts to aid the querying agent in the domain of knowledge for which it has the most interest. Thus this policy is optimistic, representing a "best bang for the buck" implementation, and works on the assumption that where there is the most amount of interest, learning a concept relation for that domain will make the most difference in the quality and effectiveness of subsequent queries. To reduce the exchange of useless information an agent will never send the same agent the same piece of concept knowledge more than once. The suggestion policy used to determine the best suggestion to offer is detailed in Algorithm 9. 


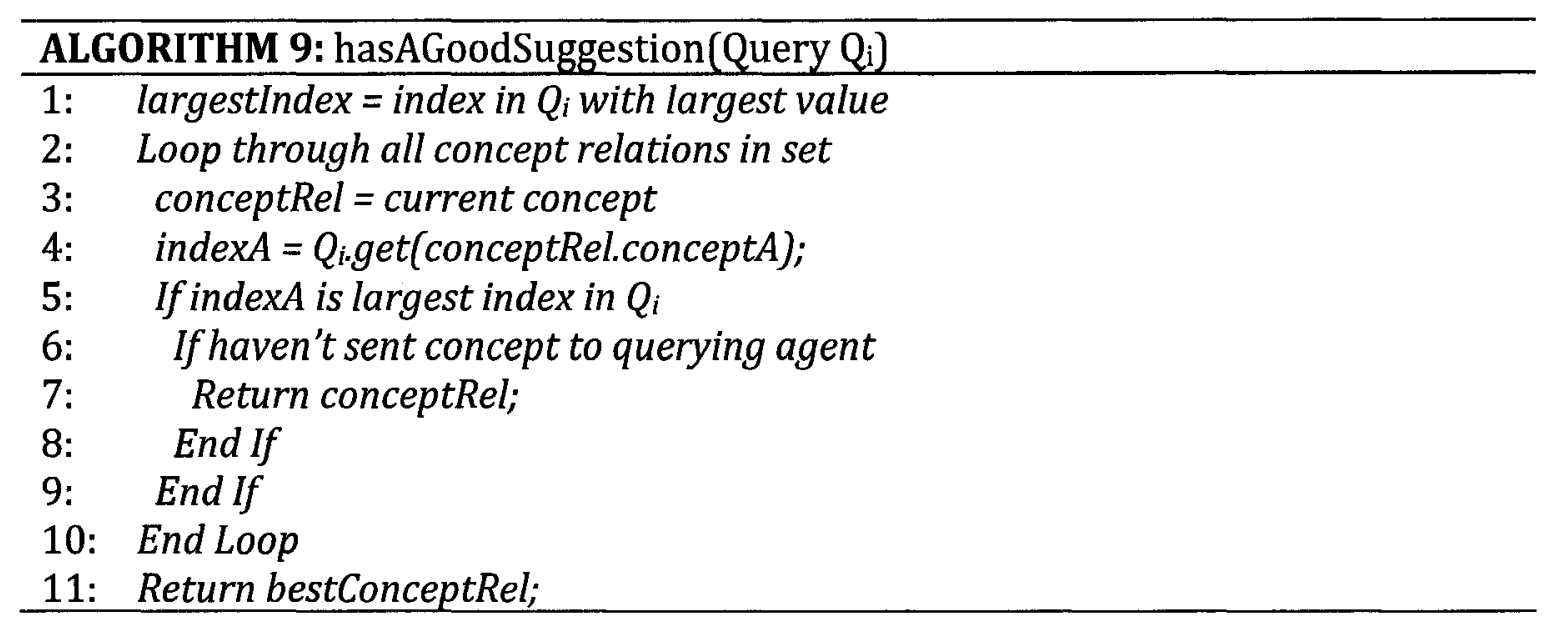

\subsubsection{Interest Adaptation Policy}

The core mechanism by which agents learn about related domains lies in the interest adaptation policy. It is responsible for adapting interests using concept knowledge and in the end determining if the concept used was helpful to the agent. If the concept is useful then trust in the agent that provided the concept knowledge is increased otherwise it is decreased. Health and trust will be used to provide this functionality and are explained further in Chapter 7. The interest adaptation policy is used when an agent has finished asking a set of questions and is learning from the responses it has received. Algorithm 1 from section 4.2 is modified in the following manner to incorporate the functionality of the Interest Adaptation Policy (line 19) and to accept incoming concept relations in the form of suggestions (line 13 to 15). 


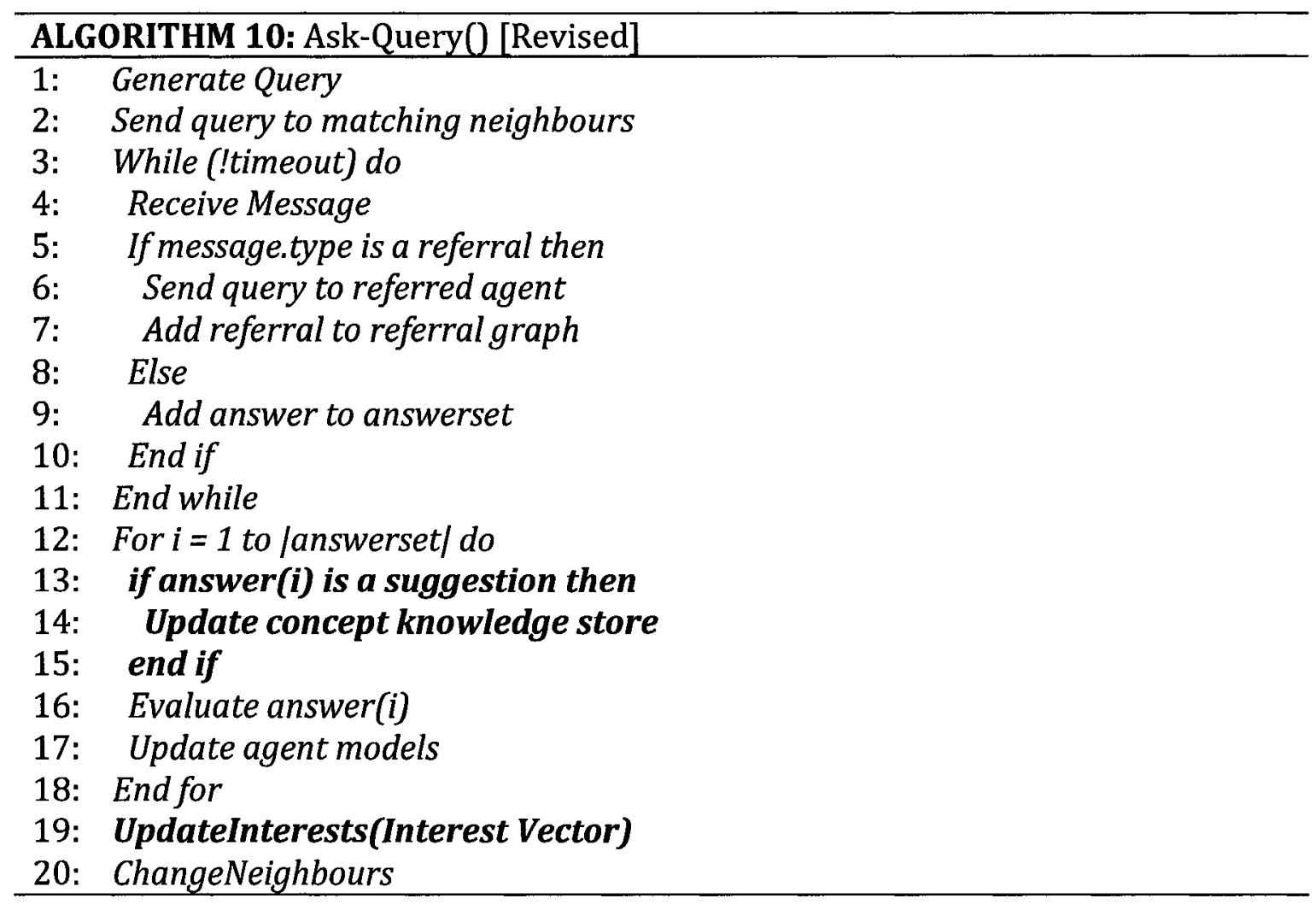

The UpdateInterests (line 19) method updates the interest of a given agent based on the Interest Adaptation policy described in Algorithm 11. When using this interest adaptation policy agents employ a goal-oriented approach. Interest in other areas is reduced (set to some small amount, in this thesis 0.01); agents are prevented from showing interest in a broad range of domains, which could potentially lead to getting fewer answers from experts in just one domain. To facilitate this goaloriented approach experts in these referral networks are only allowed to have one domain of expertise. If experts are required to have more than one area of expertise the answering policy could easily be modified in such a way that experts provide answers in only the domain which the querying agent shows the most interest. 
The interest adaptation algorithm (Algorithm 11) needs to initially determine two things -- if the agent needs to use a concept or if it should stop using the current concept (line 1). Once this has been decided, a new concept is used only if one is not currently being used (line 2); however, if the agent was using a concept it needs to determine if the concept helped the agent or not and pick a new concept to use (lines 3 through 14). Finally, interests are increased (line 17) based on the concept relation (domain B is increased).

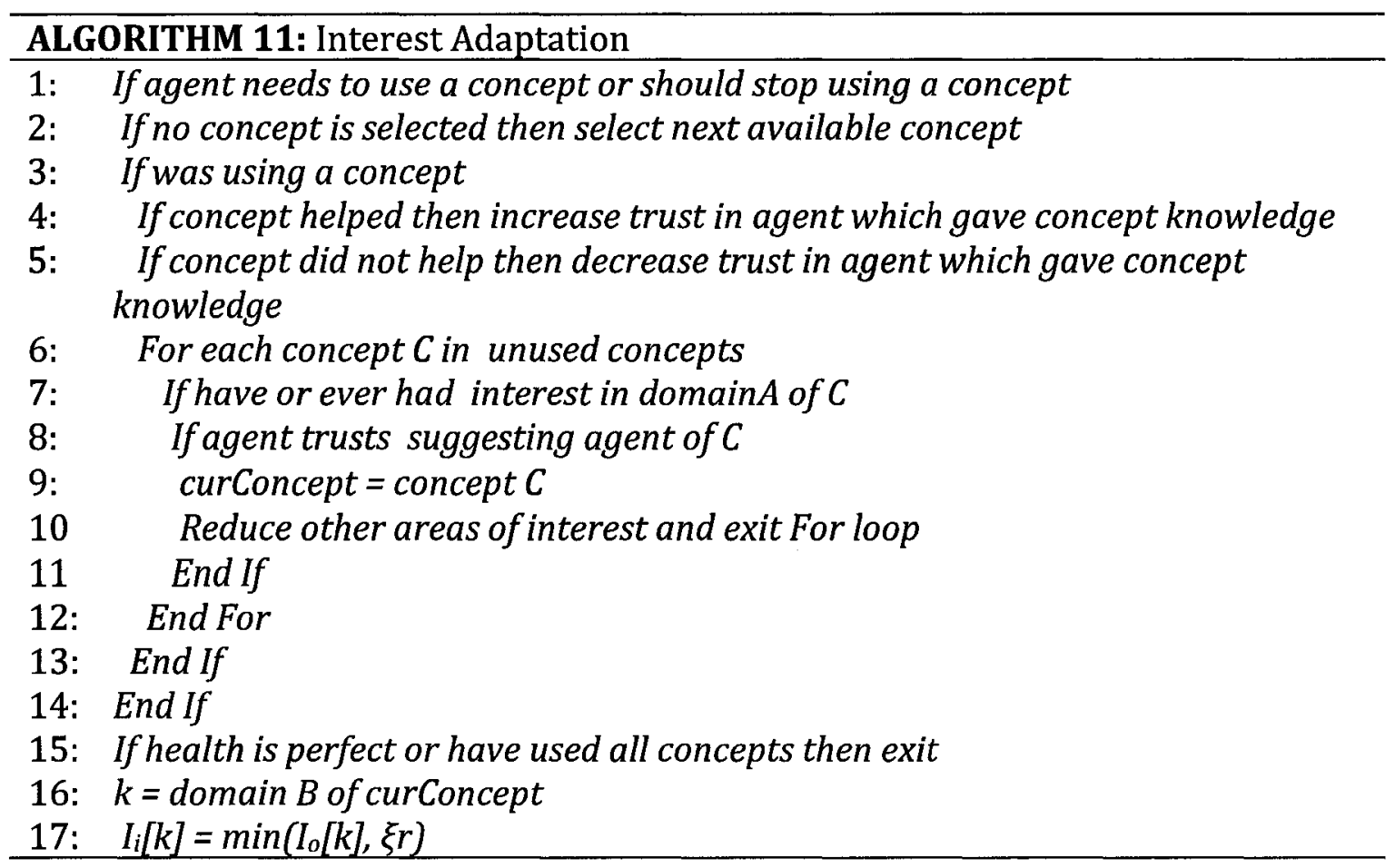

The notion of the agent needing to use or stop using a concept (line 1), and the concept helping the agent (lines 4 and 5) are left intentionally vague at this point. This algorithm will be revisited and explained in greater detail in Chapter 7, once the notions of health and trust have been concretely defined. 
The interest of agent $i$ is increased in line 17 , where $\mathrm{I}_{\mathrm{i}}$, denotes the interest vector of agent $i ; r$ is some random real number between 0 and $1, \xi$ denotes an interest increment coefficient and was set to 0.5 for all simulations, and $I_{o}$ denotes the original interest vector of agent $i$ before any interest adaptation using the current concept. Note that this calculation does not allow interest in the related domain to become larger than the amount of interest shown in the original domain. It is assumed that an agent will never want to show more interest in a related domain than in the original domain. This may be a contentious point though and is discussed further in Chapter 9.

The interest increment coefficient was set to 0.5 such that interests were increased fairly quickly as there is no penalty for showing more interest in a domain. Showing more interest will only lead to finding better experts in the network. An advantage, however, to progressively incrementing interest in this manner is that agents need not always find the best expert in the system, initially they may find moderate experts which may provide sufficient learning to satisfy the agent therefore negating the need to find a better expert.

\subsection{Referral Network Integrity}

It is important to note that the key mechanisms by which referrals and answers are evaluated and propagated in traditional referral networks remains intact. Interest adaptation only serves to modify interests and does not affect any other aspect of 
the agent's internal workings. Moreover, suggestions are not given in place of an answer or referral, but always given in addition to an answer or referral.

\subsection{Concept Knowledge Propagation}

The contribution of this thesis is highly dependent on the fact that concept knowledge can propagate to consumer agents who need it. For the concept knowledge to propagate fully it is essential that there exist a path from each consumer interested in domain $A$ of a needed concept relation to the oracle providing it. Thus it is important that consumer agents interested in domain A become neighbours with the oracle. In this way questions posed to the oracle in domain $\mathrm{A}$ will result in the concept relation for domain $\mathrm{B}$. Consumer agents interested in domain A will become neighbours of an oracle for one of two reasons; either it provides good answers or it gives good referrals when queried in domain $A$. Consequently it is important that the oracle is either an expert in domain $\mathrm{A}$ or interested in domain $\mathrm{A}$, has found experts for domain $\mathrm{A}$ and can provide quality referrals. It is conjectured in this thesis that a good strategy for placing concept relations is within the experts themselves. Thus as they provide answers for a domain they also provide suggested concept relations. In this way experts also act as oracles.

Another factor affecting the propagation of concept knowledge is the number of neighbours an agent maintains. A referral network containing agents with a larger number of neighbours will relay concept knowledge much faster. Table 1 below 
shows the number of queries it took to propagate concept knowledge to all consumer agents (360, there were 39 experts and one oracle) in a network where all consumer agents had similar interests. These numbers would no doubt be larger for increased simulation populations and where the interest landscape was more diverse, but validation of this hypothesis is considered beyond the scope of this thesis.

\begin{tabular}{|l|l|l|}
\hline Four Neighbours & Eight Neighbours & Sixteen Neighbours \\
\hline 2000 & 1600 & 1200 \\
\hline
\end{tabular}

TABLE 1: Number of Queries to Propagate Concept Relation

\subsection{Summary}

The ability for an oracle agent to provide concept relations to consumer agents allows them to move beyond having static interests and find new sources of knowledge in the network. Interest adaptation is done progressively to allow agents to find a potentially wide array of experts but agents are ultimately motivated by finding good experts and thus favour speedy interest adaptation. The propagation of concept knowledge using concept relations necessitates the need to evaluate the utility of reformulating interests. This requirement for utility evaluation leads to the concepts of health and trust that are explained in the following chapter. 


\section{Chapter 7}

\section{Health and Trust}

\subsection{Introduction}

This chapter introduces the concepts of health and trust briefly introduced in Chapter 5. Implicit in human learning is the evaluation of learned concepts. Through testing our knowledge we verify the relatedness of concepts and how well they help us; for instance, knowing about Newtonian physics will help you pass a Physics exam but not an English exam. The need for such evaluation of concept relations is also present in referral networks. The introduction of agent health serves to provide a mechanism to evaluate the learning of an agent. Health is then used as a measure of utility to establish trust in agents which provide concept relations.

\subsection{Health}

It is necessary during a simulation to deal with no actual user of the system, thus feedback on learning must be automatically provided. The health of an agent is meant to do this; it represents how well an autonomous agent is learning about required domains of knowledge. The agent's health is indirectly affected by how well the agent is adapting its interests. For example, if an agent is adapting its interests in a manner which will lead to the expertise that it requires, then its health 
will benefit, if not its health will suffer. The purpose of health in a referral network is twofold:

1. Perpetuate a reinforcement learning cycle which causes the agent to eventually adapt interests in a useful way.

2. Provide the basis for a utility metric which drives trust in other agents.

The health of an agent is modelled as the absolute difference between its current expertise vector and some target expertise vector, which is given by the human operator at the start of the simulation. The target expertise vector is essentially a "yard-stick" measure of how well an agent is learning intended concepts. As the agent learns more related concepts the health of the agent will move towards zero, indicating that it has learned all needed concepts. The equation of measuring health is given below.

$$
H_{H e a l t h}(i)=\sum_{k=0}^{z}\left|G_{i}[k]-E_{i}[k]\right|
$$

\section{EQUATION 9: Measuring Health of an agent}

In Equation 9, the target expertise for agent $i$ is represented by $G_{i}$, the current expertise of the agent is $E_{i}$, and $z$ is size of the expertise vector $E_{i}$. A health value of zero denotes perfect health, i.e., it has learned all related concepts. Any value between zero and the size of the expertise vector denotes an increasingly worse 
health rating. Health measurements are read after each query and are stored for historical analysis.

It is important to note that the target expertise is never used in any decision making process. Just as in real-life where we must first learn a concept before we can evaluate its utility and relatedness, agents must only learn of a concepts benefit after learning it and where the evaluation mechanism is an independent process to decision making. In the real world, a utility measure would either be explicitly provided by the user or implicitly through their actions.

\subsubsection{The Need for Health}

While quality measures how easily agents find useful providers it does not show how well agents are learning domains that they need to learn. An agent may have poor health yet the quality of the network is good, this situation would arise if the agent is, for instance, using a concept relation which is useless to the agent, but for which there are many experts in the network. Conversely an agent may have good health but the quality of the network may be poor. For instance, an agent may have just finished using a useful piece of concept knowledge and has learned about a needed domain, but now has moved onto a piece of concept knowledge which suggests a domain for which there are few or no experts in the network. As these examples illustrate the quality, effectiveness and authoritativeness of a network are irrelevant if agents are not actually learning about useful domains. Thus health is a 
measure to evaluate how well an agent is learning and is essential to evaluate the utility of concept relations.

\subsubsection{Health Stagnation}

Health stagnation serves as an indicator that an agent has completed learning in a given domain by examining a window of health readings. It is assumed that the agent's health will never degenerate in the lifecycle of a simulation. A method is therefore needed to determine if the health of the agent has reached a plateau and is not significantly improving. The number of health readings compared is determined by the configuration option $\mathrm{H}_{\mathrm{r}}$ (the maximum number of health readings to determine health stagnation). The health is said to have stagnated if the difference of the summation of consecutive health readings is within some threshold $\mathrm{H}_{\mathrm{t}}$. Health stagnation is determined by using Algorithm 12.

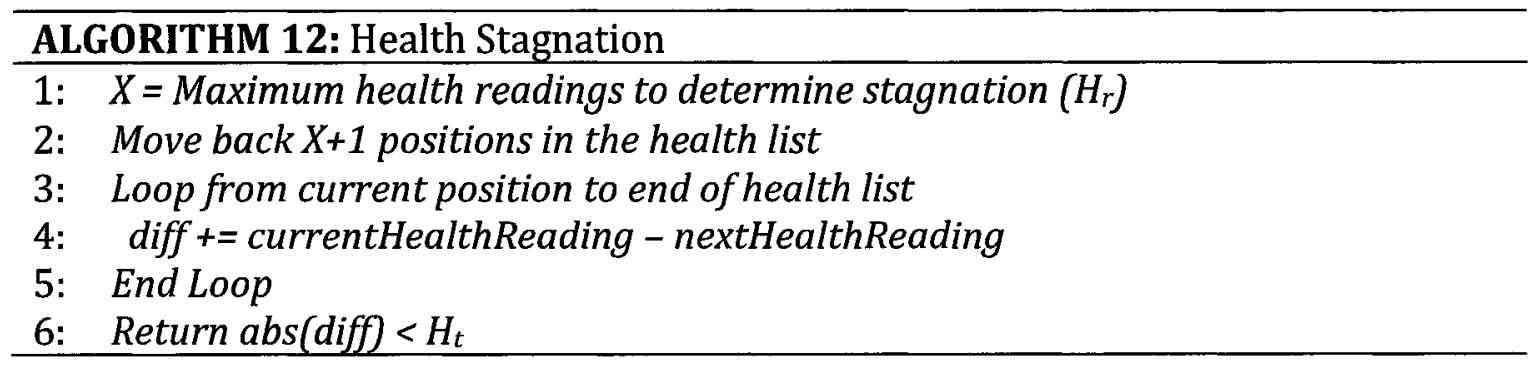

The $\mathrm{H}_{\mathrm{r}}$ variable is the determining factor for how quickly concepts are used. The smaller the value of $H_{r}$ the quicker concepts will be used. There is a trade-off, however, if $\mathrm{H}_{\mathrm{r}}$ is too small agents may not have enough time to find experts in the network and potentially useful concept knowledge will be rendered useless; 
additionally the agent that suggested the concept knowledge will be treated as untrustworthy, when, in fact, this may not be the case. If $\mathrm{H}_{\mathrm{r}}$ is too big agents will most likely find useful experts, but may take longer than necessary to realize that they have learned sufficiently. Throughout the simulations in this thesis, $\mathrm{H}_{\mathrm{r}}$ is tuned to a value of 5 to ensure that agents have sufficient time to adapt their interests sufficiently to find experts in the system; optimizing $\mathrm{H}_{\mathrm{r}}$ is not considered within the scope of this thesis and is considered future work.

The $H_{t}$ simulation variable also affects how concepts are used. A large value of $H_{t}$ and large variations in health will be considered stagnant, which is not desirable. Thus, a small value for $H_{t}$ was used (0.1) in this thesis to ensure that, over $5\left(H_{r}\right)$ successive readings the agent had learned sufficiently in a related domain. As with $\mathrm{H}_{\mathrm{r}}$, fine-tuning the precision of this value is not a significant goal of this thesis and is considered future work.

\subsubsection{Target Expertise and Learning}

To ensure that the health metric stagnates in accordance with Algorithm 5, the learning policy is modified to ensure that learning in a given domain never exceeds the target expertise for that domain. Thus line 2 in Algorithm 5 from section 4.2 is modified according to Equation 10, where $T_{i}$ is the target expertise vector for agent $i, E_{i}$ is the expertise of agent $i$, and $B_{i}$ is the best response for agent $i$, as defined in Equation 4. 


$$
E_{i}[k]=\min \left(T_{i}[k],(1-\alpha) E_{i}[k]+\alpha B_{i}[k]\right)
$$

\section{EQUATION 10: Updating Expertise}

\subsection{Trust}

Trust can be thought of as the "qualified reliance on received information." [50] Implicit in this definition is that trust must be evaluated locally in order for it to be reliable; it must provide some utility or else it cannot be relied upon. This section explains how trust is modelled and used in conjunction with health to enhance the performance of a referral network with concept knowledge.

\subsubsection{Health and Trust interaction}

Local evaluation of information (answers in a referral network) is achieved by evaluating the effect it has on the agent; i.e., its health. The measure of health can then be used to locally evaluate concept knowledge the agent has used. There is no distinction between malicious and useless concept relations; if the health of the agent gets better by using a concept relation it can then place more trust in the oracle who gave the suggestion, alternatively if the concept provided no utility less trust is placed in the oracle. Trust can then be used to determine future usage of concepts from the oracle.

The interplay of health and trust in concept knowledge form a reinforcement learning feedback loop. Concept knowledge adapts interests, which affects learning 
and increases expertise, which may or may not benefit the agent's health and thus results in an increase or decrease of trust in the agent who suggested the concept relation in the first place.

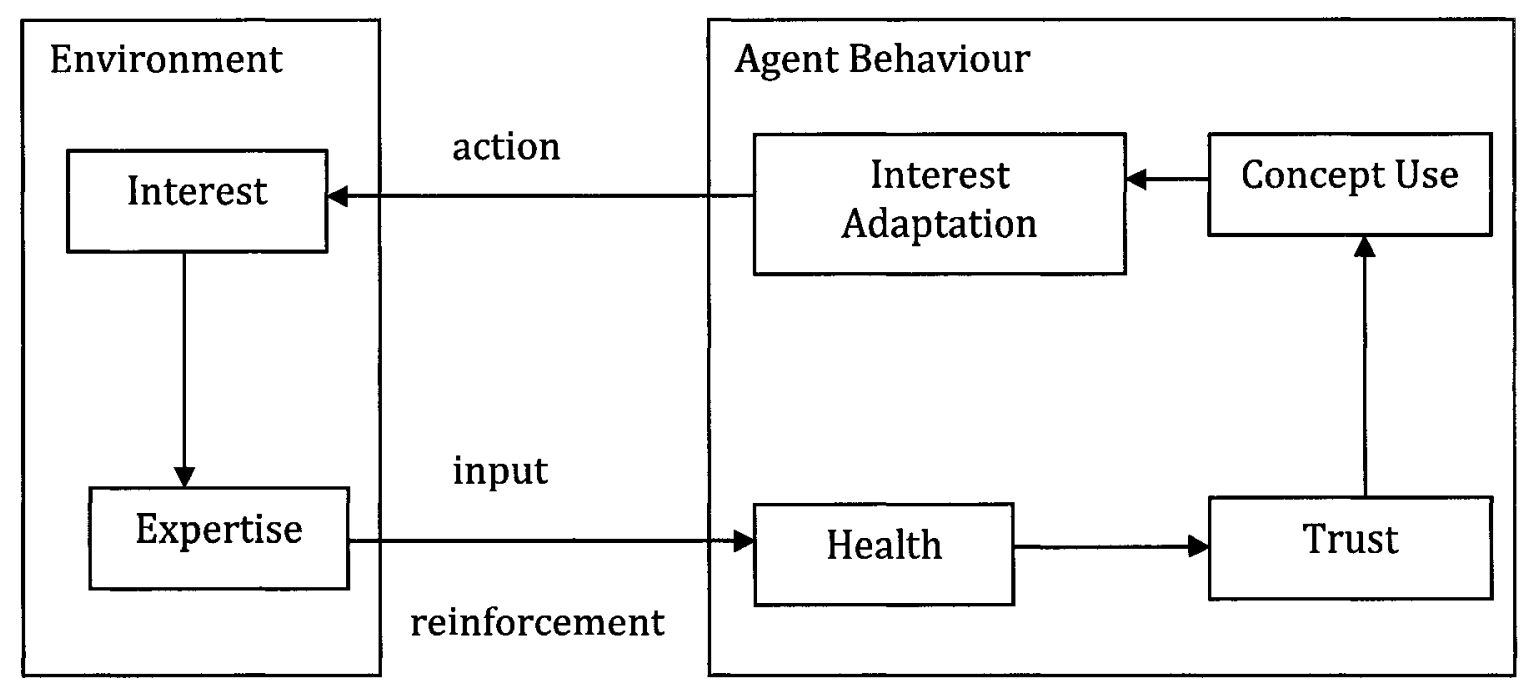

FIGURE 10: Interaction of health and trust

In the typical reinforcement model [51] the environment would be the interest and expertise of the agent, the health rating is the input and reinforcement signal indicating the state of the environment and the action is to adapt interests based on concept knowledge.

\subsubsection{Modelling Trust}

Trust is modelled as a floating point value in the range 0 to 1 , where 0 denotes full trust and 1 denotes complete distrust. Agents model trust using separate models for each agent it receives concept knowledge from during the simulation. Initially agents are naïve and put complete trust in the concept knowledge received from 
other agents. As concept relations are proven to be useful or not (based on the health metric) trust is either increased or decreased in the oracle who provided the concept knowledge. In this way trust is essentially a localized utility function for concept knowledge.

\subsubsection{Trust Functions}

Degrees of trust are calculated using trust functions. Trust functions are used within an agent to model the amount of trust (or distrust) placed in other oracles. Trust functions can be any valid mathematical monotonically increasing function which starts near or on the plane $y=0$ and approaches or intersects the plane $y=1$. An important note about the trust function data structure is that it allows the definition of start and end values for the $\mathrm{x}$-axis, thus allowing specific portions of the function curve to be used.

Three trust functions are used in the simulation results presented in Chapter 8, they are:

1. Exponential; given by $f(x)=e^{x-5}$

2. Linear; given by $f(x)=x$

3. Sigmoid; given by $f(x)=\frac{1}{1+e^{-x}}$

\subsubsection{Trust Automaton}

Trust is ultimately represented by associating an automaton with a trust function. Each agent contains a trust automaton for each oracle which provides it with a 
concept relation. Thus the level of trust of agent $i$ in oracle $j$ is defined as $T A(x)_{i j}$, where $x$ is the state of the trust automaton $T A$, and $T A(x)_{i, j}=f(x)$, where $f$ is the trust function associated with $T A$. Moving from one state to another in the automaton, results in moving along the trust function in the direction of the $\mathrm{x}$-axis. In this way the agent is either increasing or decreasing its trust in an oracle (as determined by $f(x)$ of the trust function) depending on which way it moves along the $\mathrm{x}$-axis. Increasing the automaton state will increase distrust in an oracle, while decreasing the automaton state will increase trust in the oracle (as functions are always monotonically increasing). Agents are initially naïve which means agent $i$ will assign the trust automaton $T A$ for agent $j$ to state $0\left(T A(0)_{i j}\right)$ upon receiving the first concept relation from agent $j$. The state 0 is a special case and always returns the value 0 , that is $f(0)=0$.

An example of a 10 state trust automaton with a linear trust function is given in Figure 11. As can be seen in this graph if the agent moves from state 5 to 6 , the trust function moves from a distrust rating of 0.5 to 0.6 , thus trust is decreased. 


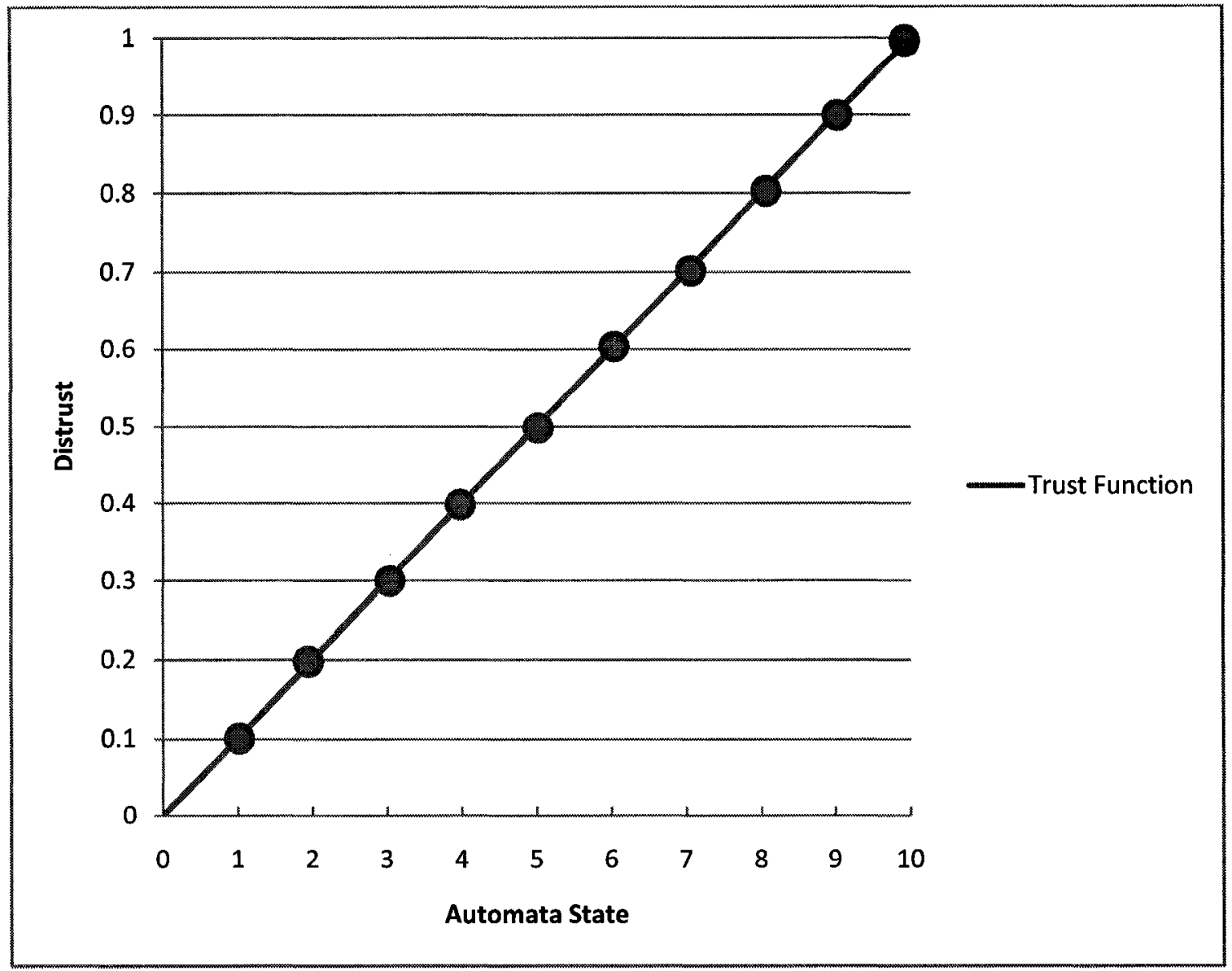

FIGURE 11: Linear Trust Automata Example

\subsubsection{Global Trust Evaluation}

The global trust of an agent is measured by taking the average value of all other agents trust ratings of the agent. Suppose that $Z$ is a set of agents that have a trust rating in the agent $i$, and $T_{i, e}$ denotes the trust of agent $e$ in agent $i$, where $e$ is an element of the set $Z$. The trust measurement of an agent $i$ would then be given by the following formula. 


$$
\operatorname{Trust}(i)=\frac{\sum_{e \in Z} T_{i, e}}{|Z|}
$$

\section{EQUATION 11: Average trust value placed in agent i.}

\subsection{Interest Adaptation Algorithm Revisited}

The agent behaviour as illustrated in Figure 10, is completely contained within the Interest Adaptation policy, and now that concept relations, health, and trust are defined, a full-fledged interest adaptation policy can be presented. The revised version of Algorithm 11, initially given in section 6.3.2, is shown in Algorithm 13.

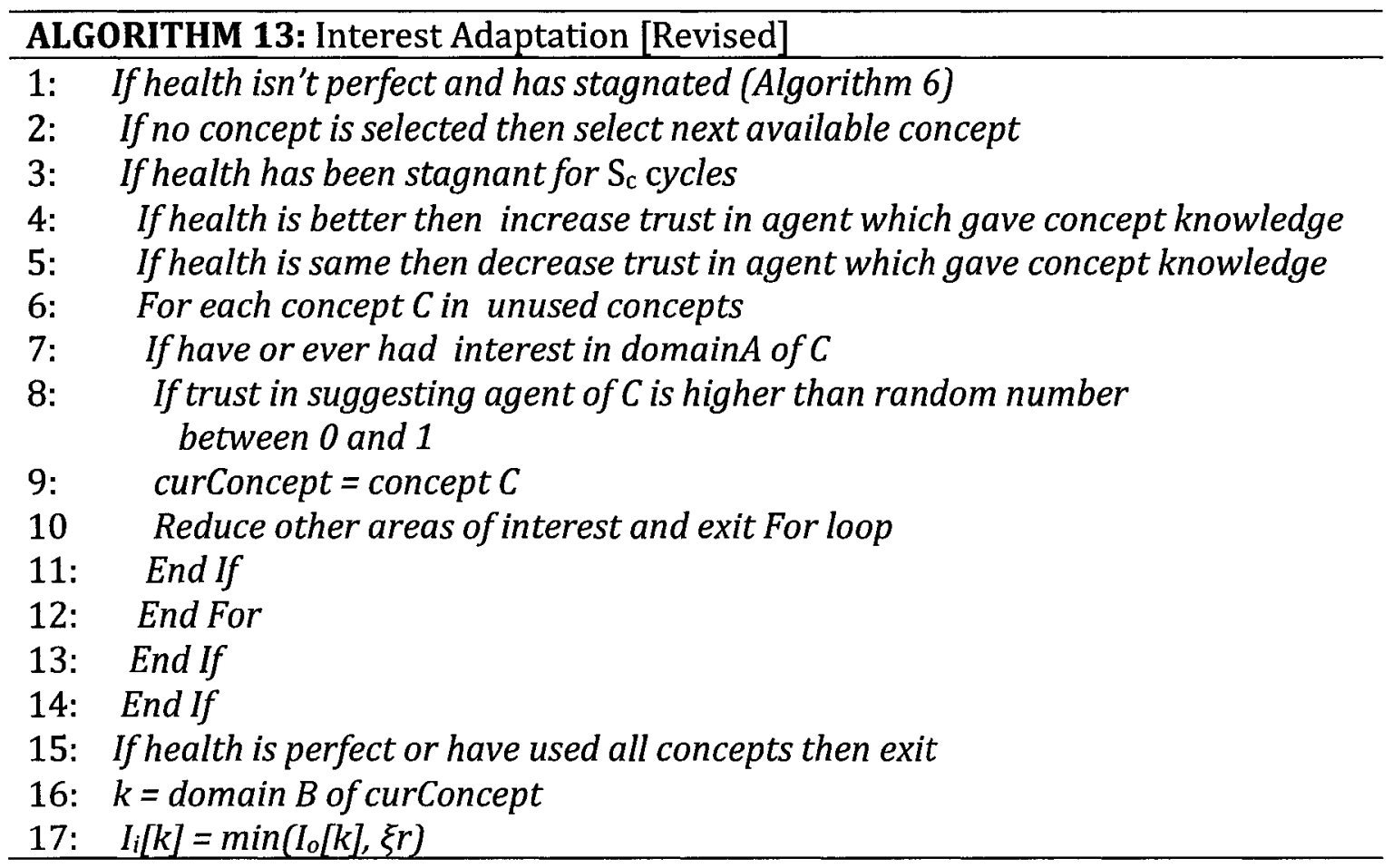

Health stagnation can now be used to determine if an agent needs to use a concept relation. If querying with the current set of interests has caused the agent's health to stagnate (line 1) the agent attempts to use the next available concept (line 2). 
However, if health has stagnated and the agent is currently using a concept relation it will attempt to use it for $S_{c}$ cycles before giving up. This is necessary as health stagnation motivates two different aspects of this algorithm; when to choose a new concept relation and when to stop using the current one. The $S_{c}$ variable was chosen as $\mathbf{5}$ for simulations in this thesis as it took no more than 5 cycles for consumer agents to find experts in the network. It is the realization of the author that this method is rather simplistic and may not be optimal in diverse simulation environments; however, the goal of this thesis is not to provide the best way to determine when to stop using a concept relation, but rather a simple and useable method. This basic scalar value could easily be replaced by a functional method where the longer a concept was used the more likely it would be decided to terminate its use; this is considered future work.

The vague notion of trust can now be replaced by a trust automaton; lines 4 and 5 can now use a trust automaton for each agent giving concept relations to represent trust. Agents will decrease the state of the automaton in line 4 (increase trust) if health is benefited and increase the state of the automaton in line 5 (increase distrust) if health is not improved. Additionally the trust automaton is used in line 8 to read the current value of the trust function as determined by the state of the automaton, and determine if an agent is trustworthy enough to use their suggested concept relation. 


\subsection{Summary}

Evaluating how well an agent is learning is essential to building trust in how the agent is learning. This chapter introduced the concept of agent health which is a measure of how well the agent is learning needed concepts based on some predefined target. The notion of localized trust automaton functions is also presented. Health acts as a reinforcement signal to motive trust/distrust in oracle agents that share concept knowledge. With interest adaptation, concept suggestions, health and trust in place, simulations are presented in the next chapter illustrating the power of referral networks with relational concept knowledge. 


\section{Chapter 8}

\section{Simulation Setup and Results}

\subsection{Introduction}

This Chapter presents the setup and results of five simulations intended to:

1. Explore a referral network configuration which allows the formation of expert communities

2. Examine the effect of oracles; i.e., concept relations and suggestions

3. Examine the effect of learning rates

4. Determine success of local trust using trust automata functions

5. Demonstrate the ability to distinguish subjective context and transitive concept learning.

Comprehensive details on the simulation setups can be found in Appendix A.

\subsection{Baseline Simulation}

This simulation is meant to evaluate baseline measures of traditional referral networks compared to referral networks with concept knowledge. To this end three referral networks were created each with 400 agents. The first was a traditional referral network with 40 experts in domain 0 and the remaining 360 consumers with interest in domain 0 . The second was also a traditional referral network with 360 consumers interested in domain 0 , but the 40 experts now had expertise in 
domain 1. This is meant to represent a community where the experts are in a domain related to the interests of the consumers, but there exists no means for the consumers to find or utilize these experts. The third referral network contained 39 experts in domain 1, 360 consumer agents interested in domain 0 and one oracle to show the consumers that domain 0 was related to domain 1 . Thus the oracle contained the concept relation $0 \rightarrow 1$. In all three referral networks the experts were allowed to have a high interest in the area of their expertise and thus would seek out other experts like them (they formed expert communities). Further information on simulation setup is presented in Appendix A.

In this simulation and the others presented in subsequent sections, the expert and consumer populations are homogenous in terms of their domains of interest and expertise. This was done intentionally as it is the belief of this author that a central tenet of social search is asking questions specific to an area or domain. We do not ask an expert questions based on a complete set of our interests but rather the most specific set possible and the ones we are currently interested in pursuing. As an example, if I am currently interested in understanding NP completeness, I would not ask about NP completeness and beat-matching dance records, even though mixing records is an interest of mine.

Summarily, this simulation is intended to demonstrate that:

1. A different set of results for effectiveness and efficiency as experts are now only in one domain and are allowed to become neighbours of like experts 
2. Concept knowledge allows a referral network filled with differing providers and consumers to perform on par with one that is filled with similar providers and consumers, given that the providers are experts in a related domain, and useful concept knowledge is present.

The results for effectiveness (quality), efficiency (accuracy) and authoritativeness of the baseline simulations are presented in the subsequent sections.

\subsubsection{Effectiveness/Quality}

The results for quality are different from Yolum's results in [1]. Rather than a monotonic increase in quality as the threshold to refer is increased a decrease in quality is seen. As the threshold to refer increases the direct quality of the network is decreased as consumer and expert agents do not converge quickly to other experts. With a smaller number of referrals agents have fewer choices in terms of experts to choose as neighbours, and thus end up becoming neighbours with fewer experts. This lack of convergence also leads to a decrease in the $\mathrm{n}^{\text {th }}$ best quality metric. Since each of the experts themselves will have fewer experts as neighbours, this means that any agent will now have to follow a longer path, on average, to any given expert in the network. Thus the $\mathrm{n}^{\text {th }}$ best metric, which largely depends on proximity in the network, will be decreased.

These results contrasts referral networks where experts form smaller communities (or none at all) in which convergence to an expert is essentially a "dead-end" and a 
path to other experts in the system is longer or does not exist at all (see section 5.2.1). Figure 12 , shows direct and $\mathrm{n}^{\text {th }}$ best quality over varying thresholds to refer. Curves with the label "(Normal)" represent results for the first referral network, ones with the label "(with $\mathrm{CK}$ )" represent results for the second referral network (CK stands for Concept Knowledge) and lastly curves with the label "(without CK)" represent the third referral network. Curves with the label "Nth Best" refer to the $\mathrm{n}^{\text {th }}$ best quality metric as described in section 5.2 , while curves with the label "Direct" refer to direct quality, also described in section 5.2 .

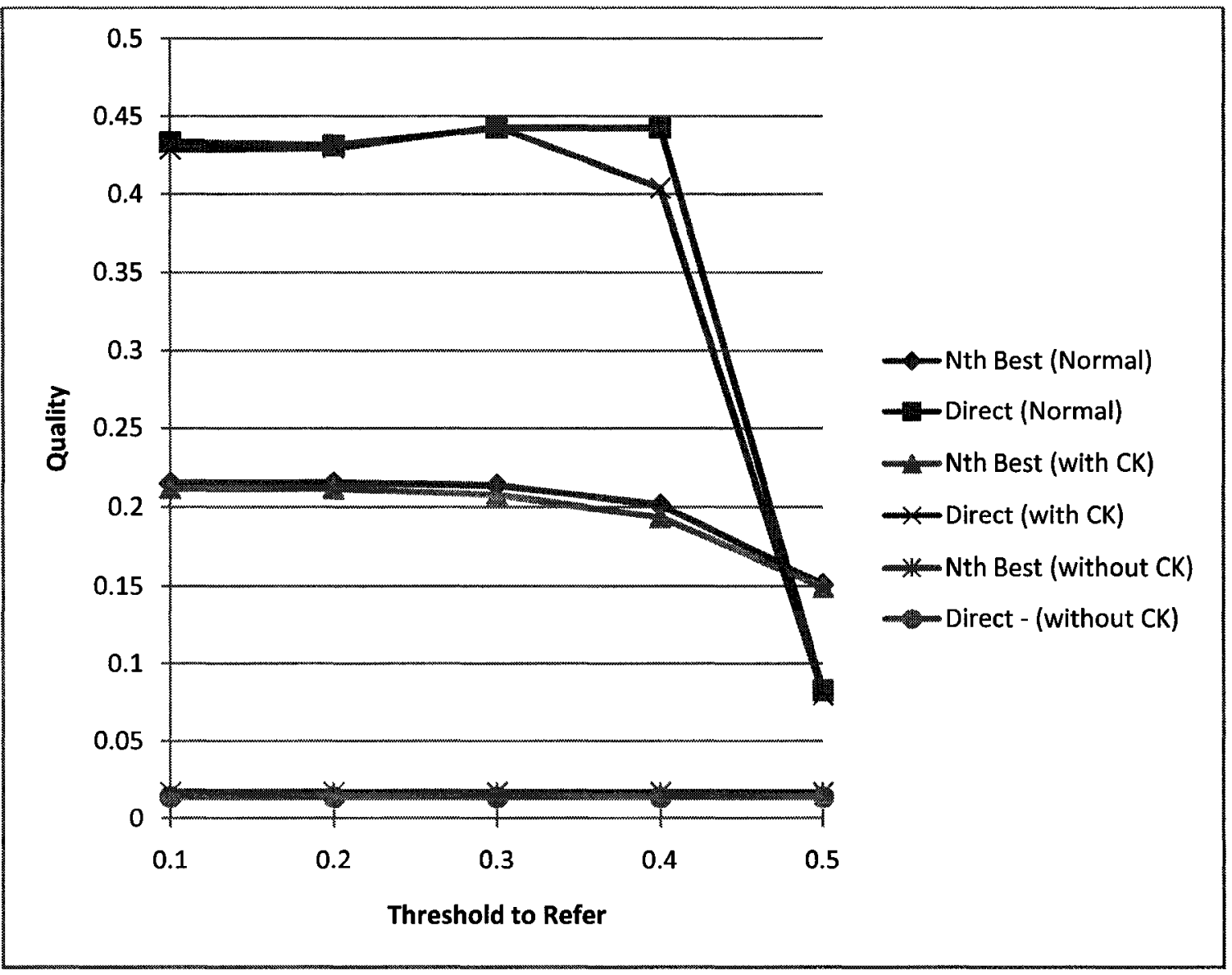

FIGURE 12: Direct and $N^{\text {th }}$ Best Quality 
It is, of course, important to note that the referral network with concept knowledge rivals the normal network and outperforms the referral network with no concept knowledge as expected.

\subsubsection{Efficiency/Accuracy}

In Yolum's work the selectivity of referrals tends to decrease accuracy as not enough experts are found in the network. Higher thresholds reduce the number of referrals and leads to less convergence on experts in the system. As fewer of an agent's neighbours are experts the number of agents contacted drops significantly leading to the increase in accuracy. This contrasts with Yolum's results where neighbours were contacted even if they could not provide answers. Figure 13, shows accuracy as measured over varying thresholds to refer. The curved labelled "Normal" refers to the first referral network, "With CK" refers to the second and "Without CK" refers to the third referral network. 


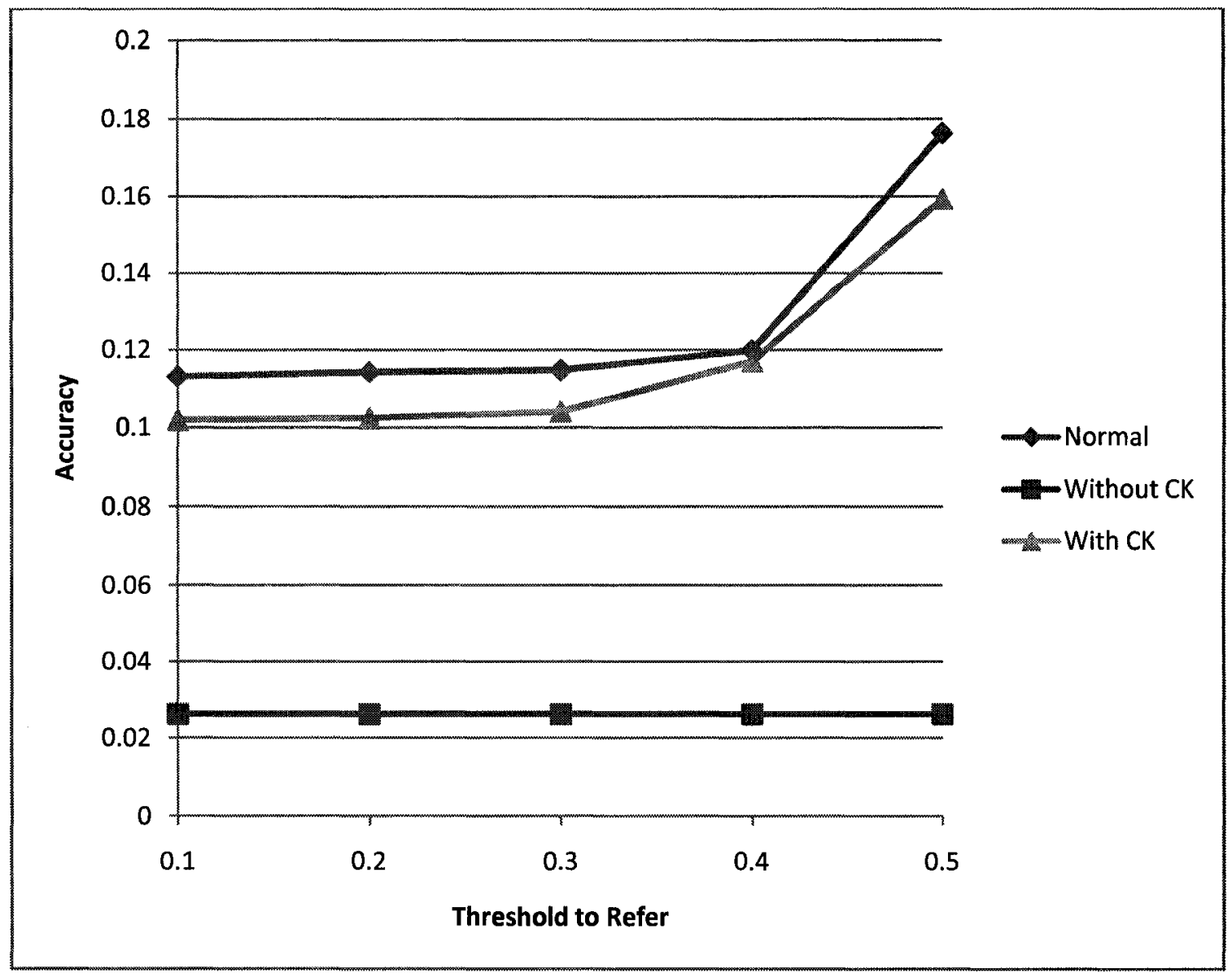

FIGURE 13: Accuracy

\subsubsection{Authoritativeness}

The emergence of authorities for referral networks with concept knowledge and with the formation of expert communities is strikingly similar to Yolum's results. Figure 14 plots the cumulative PageRank of agents in the third referral network (with concept knowledge). Three curves are plotted, for thresholds $0.1,0.3$, and 0.5 . The points represent the number of agents with a PageRank greater than or equal to the given PageRank on the x-axis. For example, 52 agents have a PageRank of 1.5 or higher when the threshold to refer is set to 0.5 . 


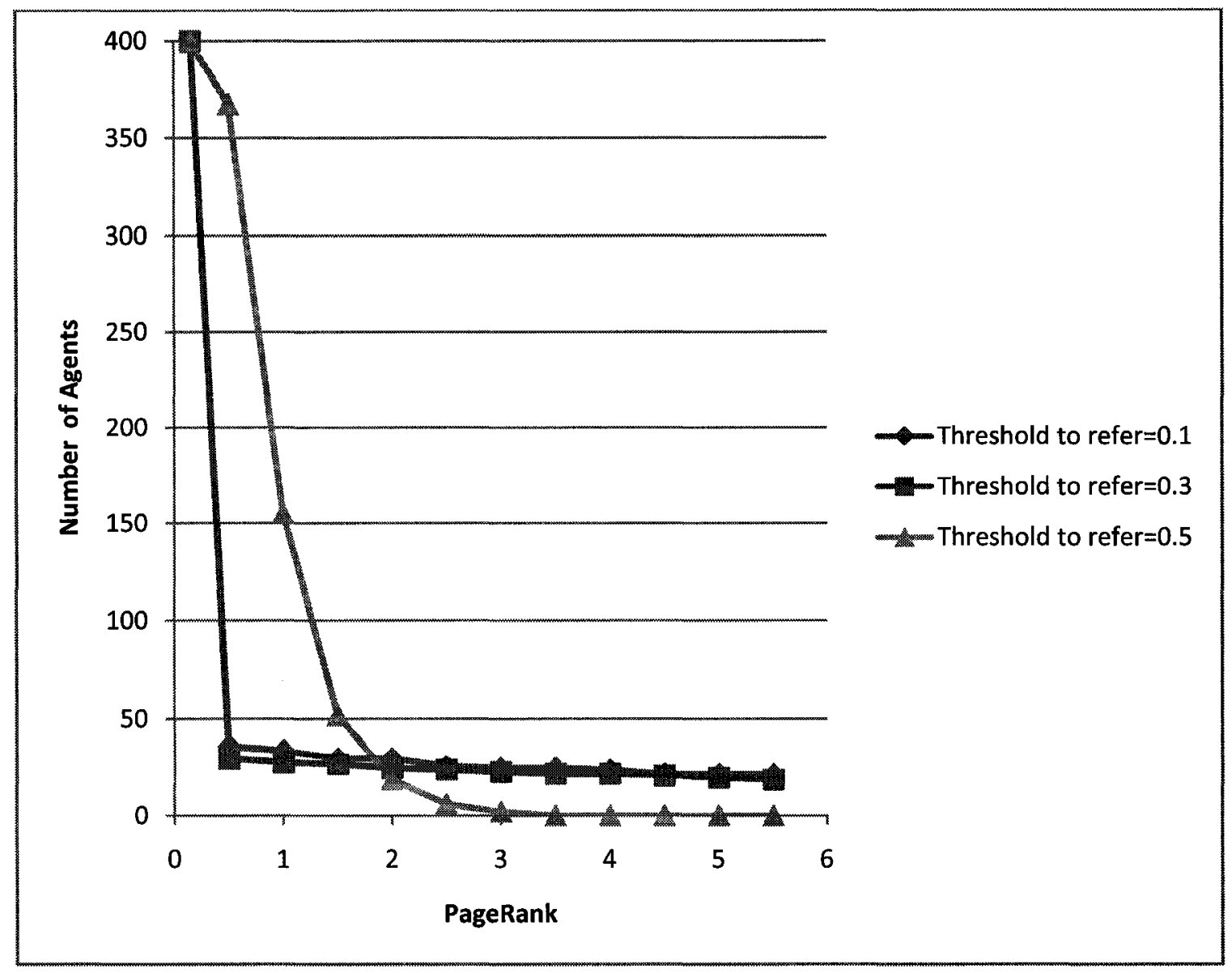

FIGURE 14: Cumulative PageRank with Concept Knowledge

Figure 14 shows that there is more convergence at lower thresholds as indicated by a larger number of the higher page ranks and the similarity in the curves for thresholds 0.1 and 0.3. However, as the threshold is increased fewer agents are more authoritative; no agent had a PageRank of larger than 3.5 when the threshold to refer was set to 0.5 . This is also found in Yolum's work, and is due to the fact that fewer referrals are given to experts in the network, when the threshold to refer is more restrictive. The results for the first referral network (traditional) are similar to these results below, and were left out for the sake of succinctness. 


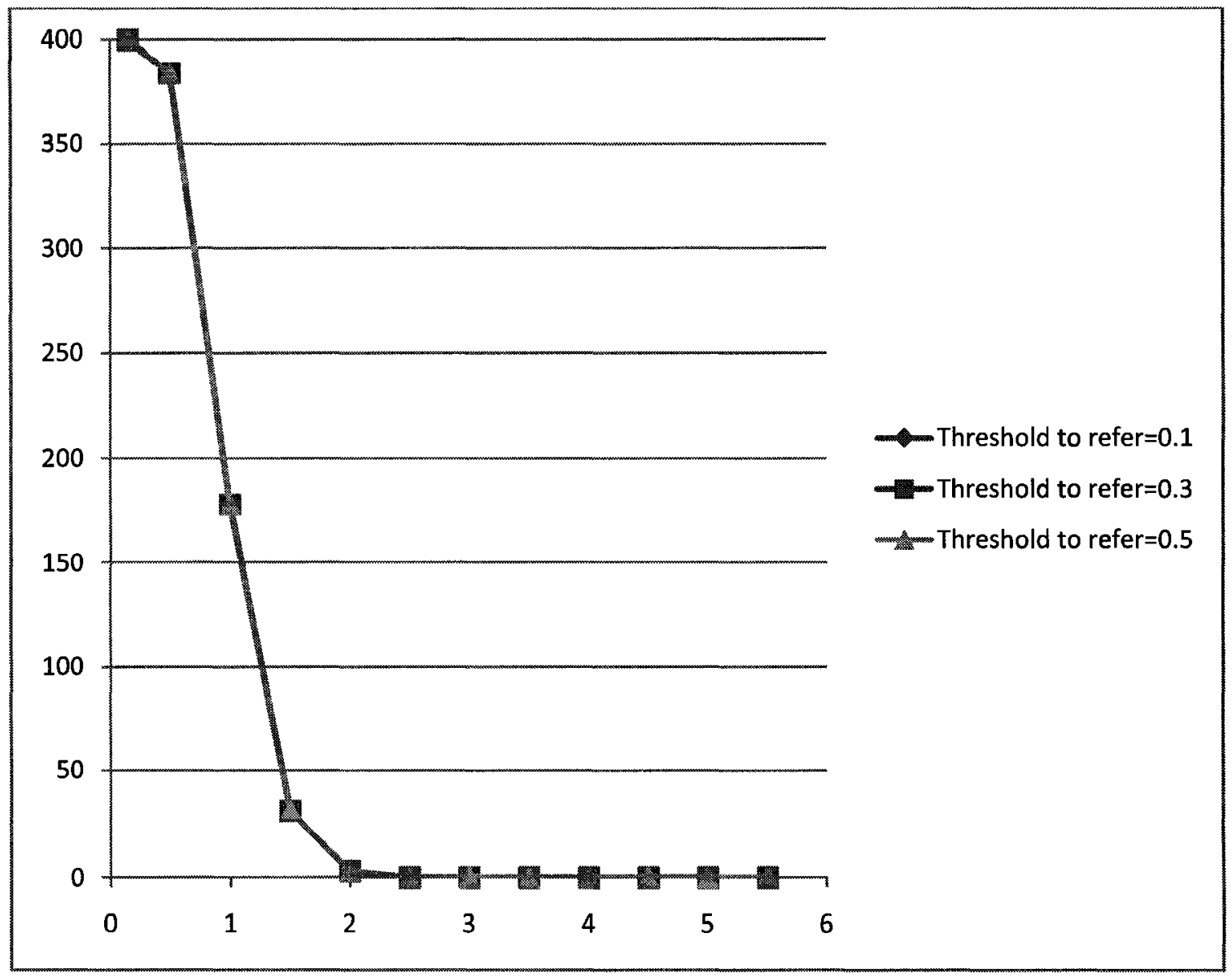

FIGURE 15: Cumulative PageRank with no Concept Knowledge

The results for referral networks 1 and 3 contrast with those of the referral network with no concept knowledge. The PageRanks are evenly distributed and insensitive to the threshold to refer and show no emergence of authority, as shown in Figure 15. There are no agents with a PageRank higher than 2, while the referral network with concept knowledge, even with a high threshold to refer (0.5) saw the emergence of authorities with PageRanks of 3. This result is to be expected though, as none of the agents are asking questions which can be answered by experts in the network, thus no clear authorities emerge. 


\subsubsection{Concept Propagation}

It is important to show that concept propagation occurs quickly and that all consumer agents receive the concept relations. Table 2 below shows that after each agent had performed their third query, all consumer agents had received the concept.

\begin{tabular}{|l|l|l|}
\hline Query Number & Concepts Shared & Agents without concept \\
\hline 1 & 29 & 372 \\
\hline 2 & 631 & 119 \\
\hline 3 & 2354 & 39 \\
\hline
\end{tabular}

TABLE 2: Concept Propagation

Concept knowledge is propagated this quickly as there exists a path from the oracle to each of the consumers. Note that the 39 agents without concept knowledge are the experts, as they never show interest in domain 0 , thus never learn about the concept.

\subsection{Effect of Learning}

Experimentally this simulation is exactly the same as the baseline simulation outlined in Section 8.2, with the notable difference that three values were used for the learning coefficient $0.05,0.15$, and 0.25 .

This simulation was intended to:

1. Ascertain the effects of learning on the quality, efficiency and authority of referral networks. 
2. Show increases in the health of agents who have learned and determine the best methods to learn.

The results for effectiveness (quality), efficiency (accuracy), authoritativeness and agent health are presented below.

\subsubsection{Effectiveness/Quality Results}

The effect of learning on direct and $\mathrm{n}^{\text {th }}$ best quality in the normal network is quite profound. As agents are allowed to learn about their domain of interest they too become experts, which causes quality to be stabilized across increasing thresholds. Figure 16 shows the effect of a learning coefficient of 0.05 on the quality of all three referral networks. Note, that the $\mathrm{n}^{\text {th }}$ best metric is consistently less than the direct measure as it takes into consideration the distance between agents.

When comparing Figure 16 to Figure 12 in section 8.2.1 there is a noticeably smaller drop in quality for higher thresholds $(0.4$ and 0.5$)$. This is due to the fact that consumer agents themselves are learning and gain some amount of expertise themselves, thus learning allows the formation of new experts and thus acts to mitigate a sharp drop in quality. 


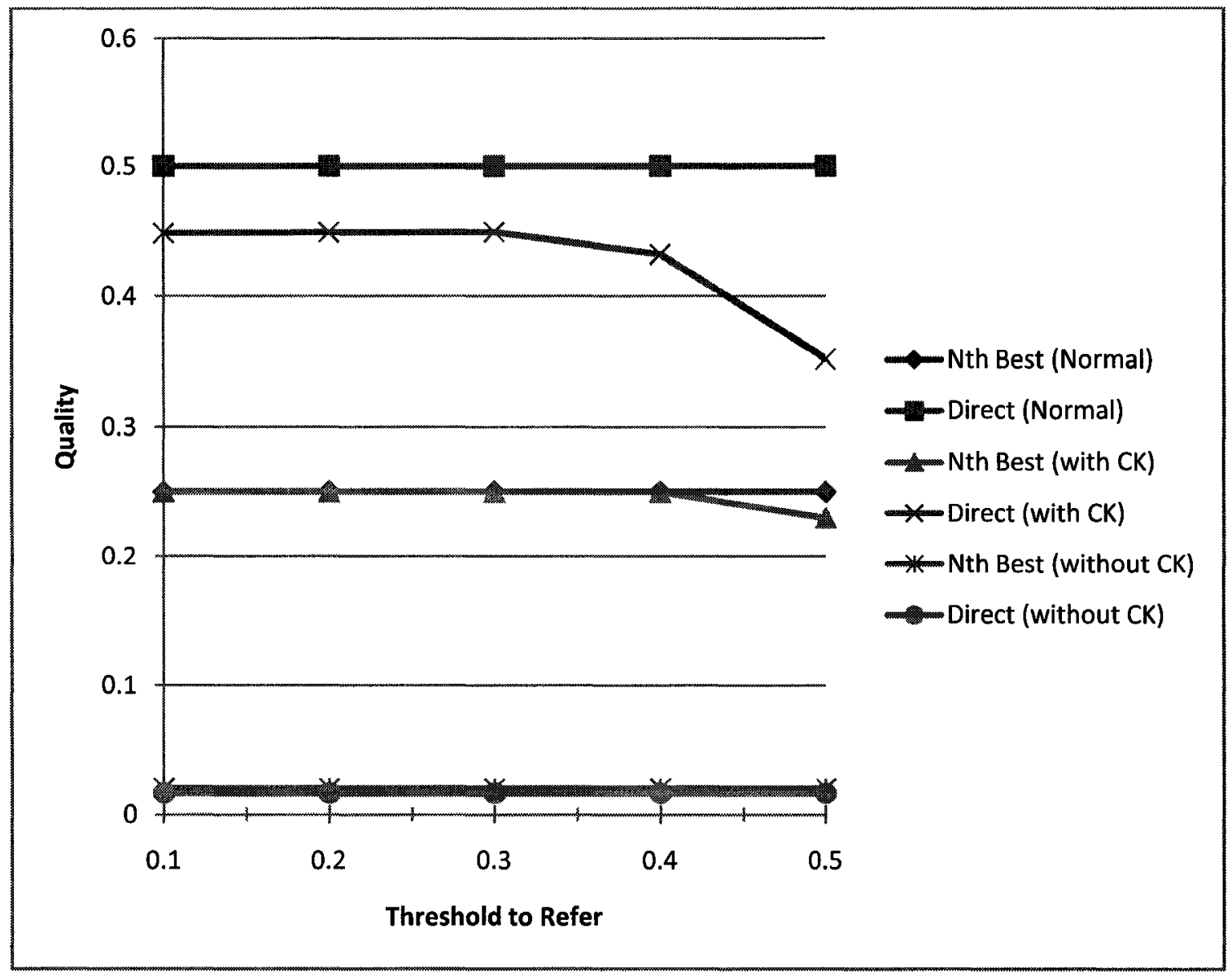

FIGURE 16: Effect of Learning on Quality ( $\alpha=0.05)$

There exists a notable difference between the direct quality metric for the referral networks with concept knowledge and the normal referral network. The direct quality for the normal network remains constant across thresholds while the network with concept knowledge is considerably lower and decreases with a higher threshold to refer. This is due to the fact that agents were tuned in the normal simulation to learn their domain of interest completely (to a value of 1.0) while in the concept simulation agents only learned marginally (to a value of 0.65 ) about the related concept. Since consumer agents in the concept referral network become at most moderate experts, the direct quality metric cannot achieve the same results as 
when they become strong experts, as in the normal network. Additionally, with a higher threshold to refer and a lower overall expertise, there are a smaller number of referrals to experts and thus agents find fewer in the system. With a smaller number of experts as neighbours the direct quality is affected.

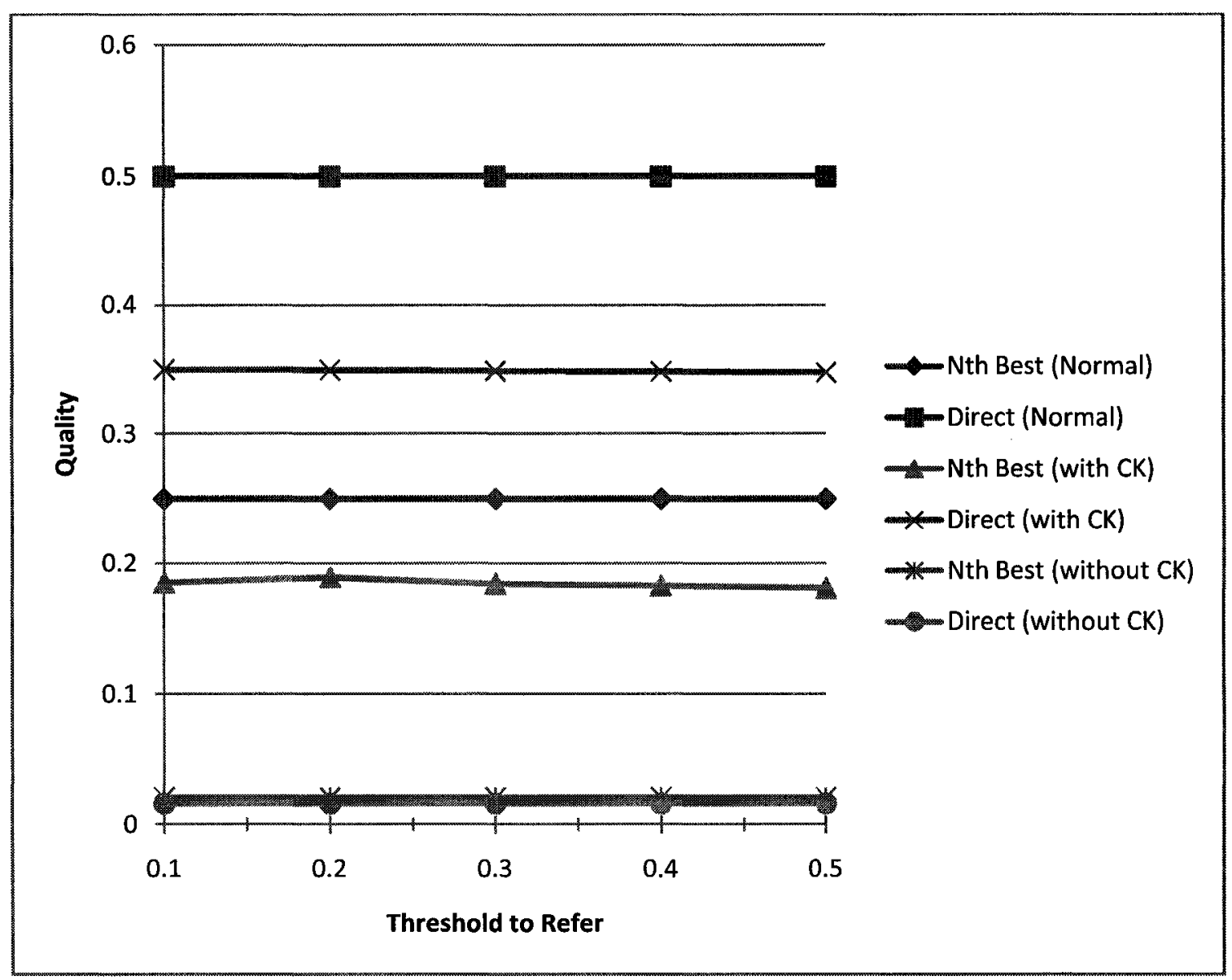

FIGURE 17: Effect of Learning on Quality ( $\alpha=0.25$ )

When the learning coefficient is increased to 0.25 , the quality for the referral network with concept knowledge tends to stabilize across all thresholds to a value less than the worst quality with $\alpha=0.05$. This indicates that as agents learn faster fewer referrals are exchanged, and therefore better experts in the system are found 
less frequently. Fewer referrals are exchanged because as agents learn faster they provide answers instead of referrals. Without finding the best experts in the system the quality of the network suffers. Figure 17 shows the effect of increasing the learning coefficient to 0.25 .

CONCLUSION. Unless consumer agents are allowed to become experts to at least the degree of the best experts in the system, it is better to learn slowly and allow referrals to find better experts in the network, if a greater degree of quality is desired.

\subsubsection{Efficiency/Accuracy Results}

It would be expected that as more agents learn about their domains of interest that the accuracy of queries is increased. The experimental results paint a different picture with accuracy being quite stable across thresholds and not performing much better than with no learning (see section 8.2.2). The accuracy rate does not increase with the threshold to refer as it did when the learning rate was 0.

When no learning was employed (section 8.2.2) fewer of an agent's neighbours were experts and thus the number of agents contacted dropped significantly with higher thresholds, leading to the increase in accuracy. However, as consumer agents become experts in the network, more of any given agent's neighbours will be experts and able to provide an answer. Thus an agent will send more of its neighbours its current query, and the overall number of requests (agents contacted) 
is increased. This tends to decrease the ratio of correct answers to total answers as the denominator is much larger than when learning is not employed and fewer agents are contacted. Thus the accuracy metric stagnates as illustrated in Figure 18. Increasing the learning coefficient to 0.15 and 0.25 produced similar results, which are not included here in the interests of brevity.

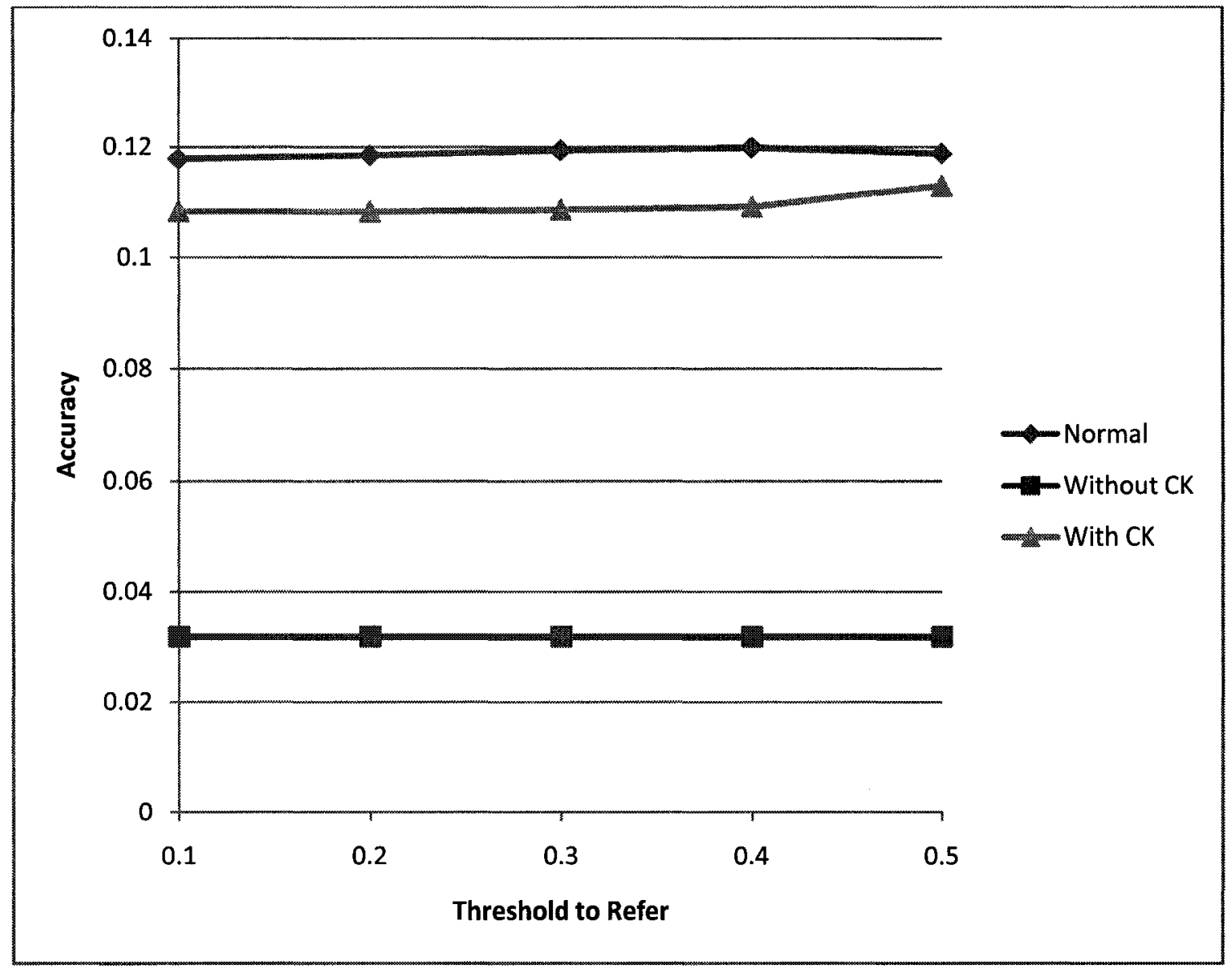

FIGURE 18: Effect of Learning on Accuracy $(\alpha=0.05)$ 


\subsubsection{Authoritativeness Results}

As more agents are allowed to learn from their responses it would be expected that a larger number of authorities emerge in the network.

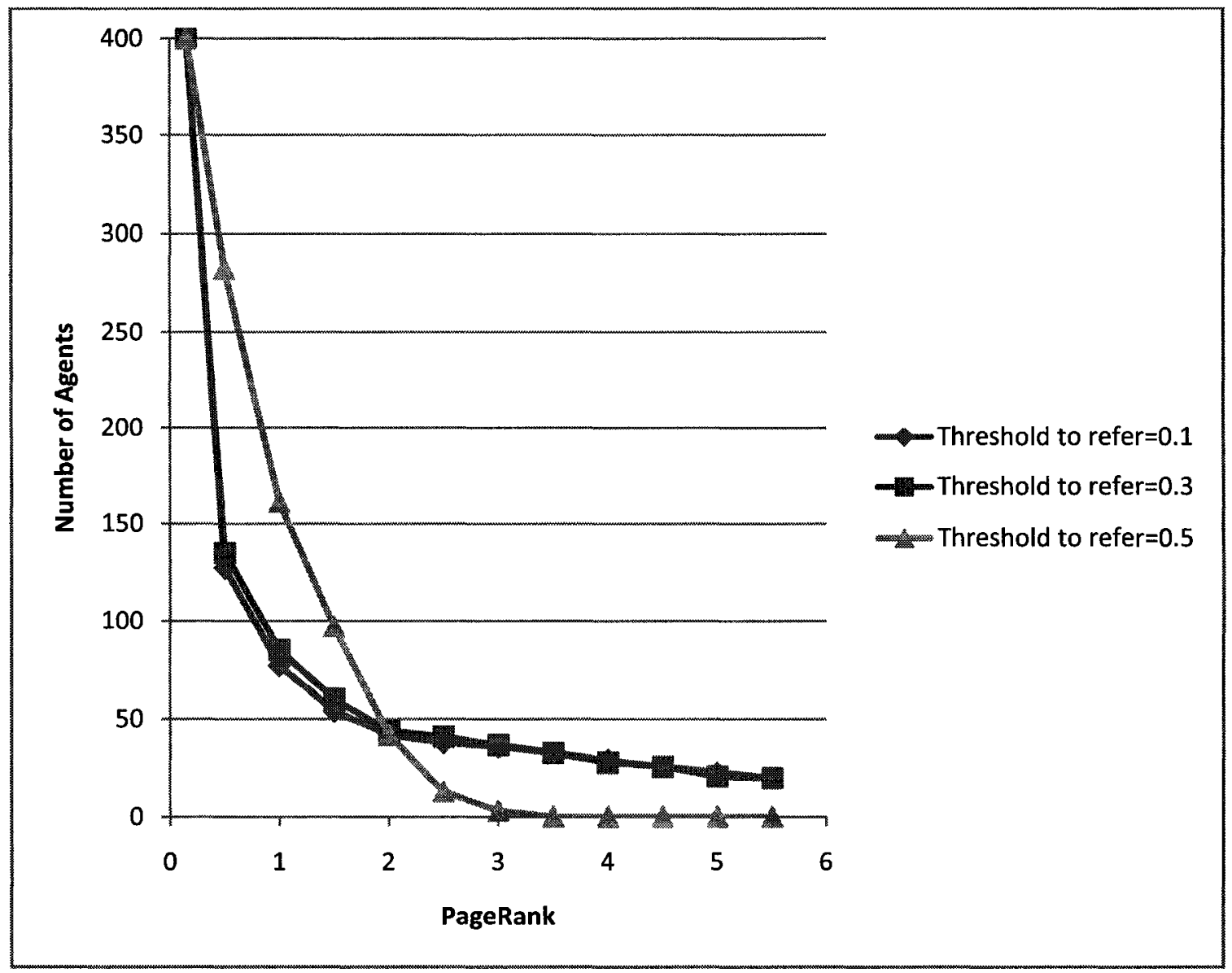

FIGURE 19: Effect of Learning on Authoritativeness ( $\alpha=0.05$ )

The cumulative PageRank is shown in Figure 19 for a normal referral network where the learning coefficient is set to 0.05 . A referral network with concept knowledge exhibits the same behaviour as a normal referral network, while a referral network with no concept knowledge attains similar results to those with no learning, as seen in section 8.2.3. 


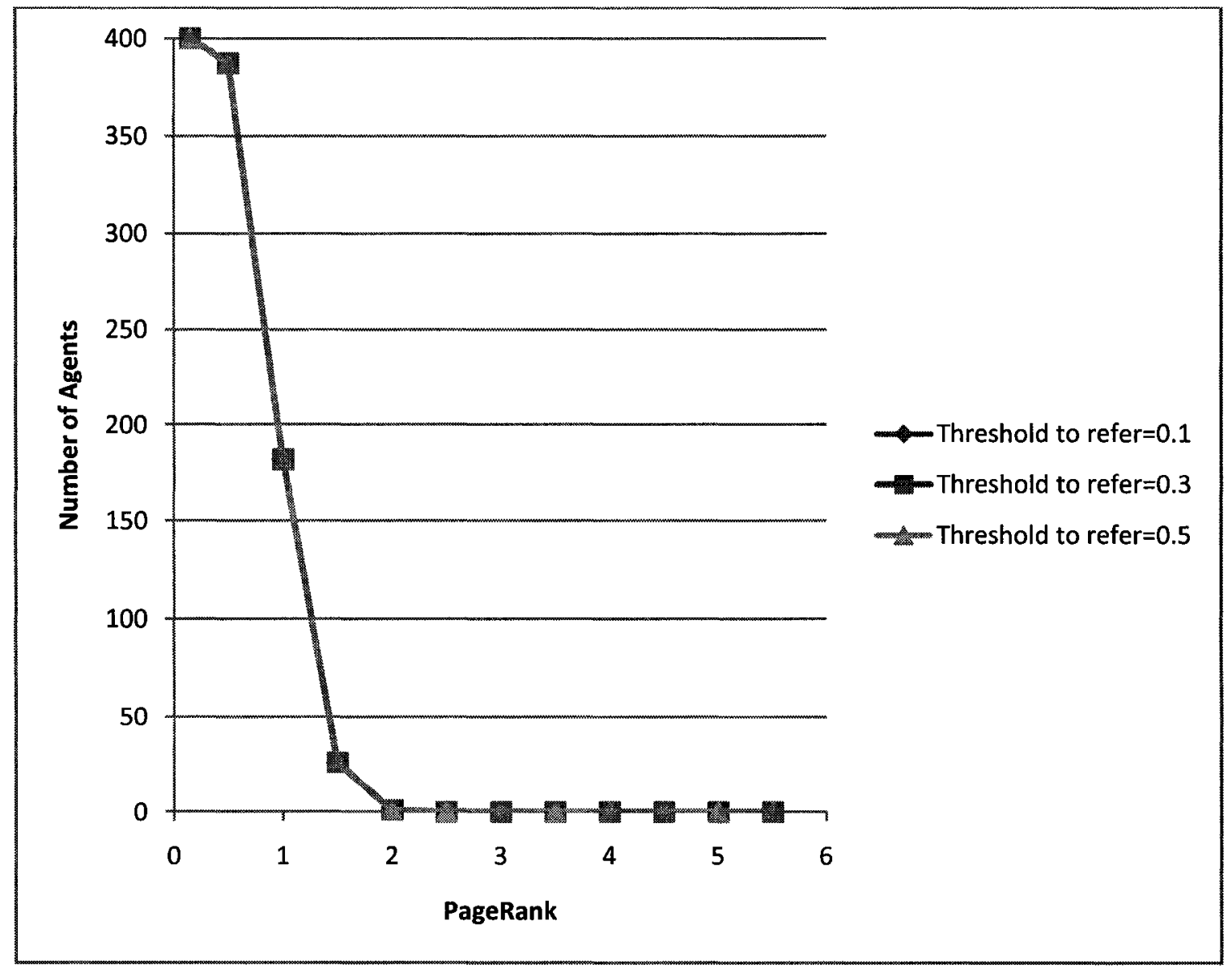

FIGURE 20: Effect of Learning on Authoritativeness ( $\alpha=0.25$ )

All thresholds have a greater number of authorities than when no learning is used (see section 8.2.3). However, an interesting result is obtained when the learning coefficient is increased further. As agents learn faster fewer referrals are given as they can provide answers. Thus fewer authorities emerge and the PageRanks across thresholds become very similar. The results for a normal referral network with a learning coefficient of 0.25 are shown in Figure 20, and are quite similar to the results when the learning coefficient is set to 0.15 . Again similar results are achieved with a referral network with concept knowledge. 
CONCLUSION. As agents learn faster, authority is dispersed over the network as agents provide answers instead of referrals to experts in the network.

\subsubsection{Learning and Health}

Since the health of an agent is intrinsically tied to what an agent is learning, it is useful to see how learning affects the health of an agent. In a normal referral network as agents learn their health increases (moves toward 0) with increasing speed as the learning coefficient is increased, which is an expected result. This also happens in the referral network with concept knowledge. To this end the results for only one of the learning coefficients is shown below (0.15). The agent's health results for all thresholds are shown for a referral network with concept knowledge in Figure 21. A referral network with a lower threshold to refer causes agents to learn faster as more referrals are made and agents become neighbours of experts more quickly. With a threshold of 0.1 agents finish learning at query number 15 , at threshold 0.2 query 16 , and thresholds $0.3,0.4$, and 0.5 at query 18 . 


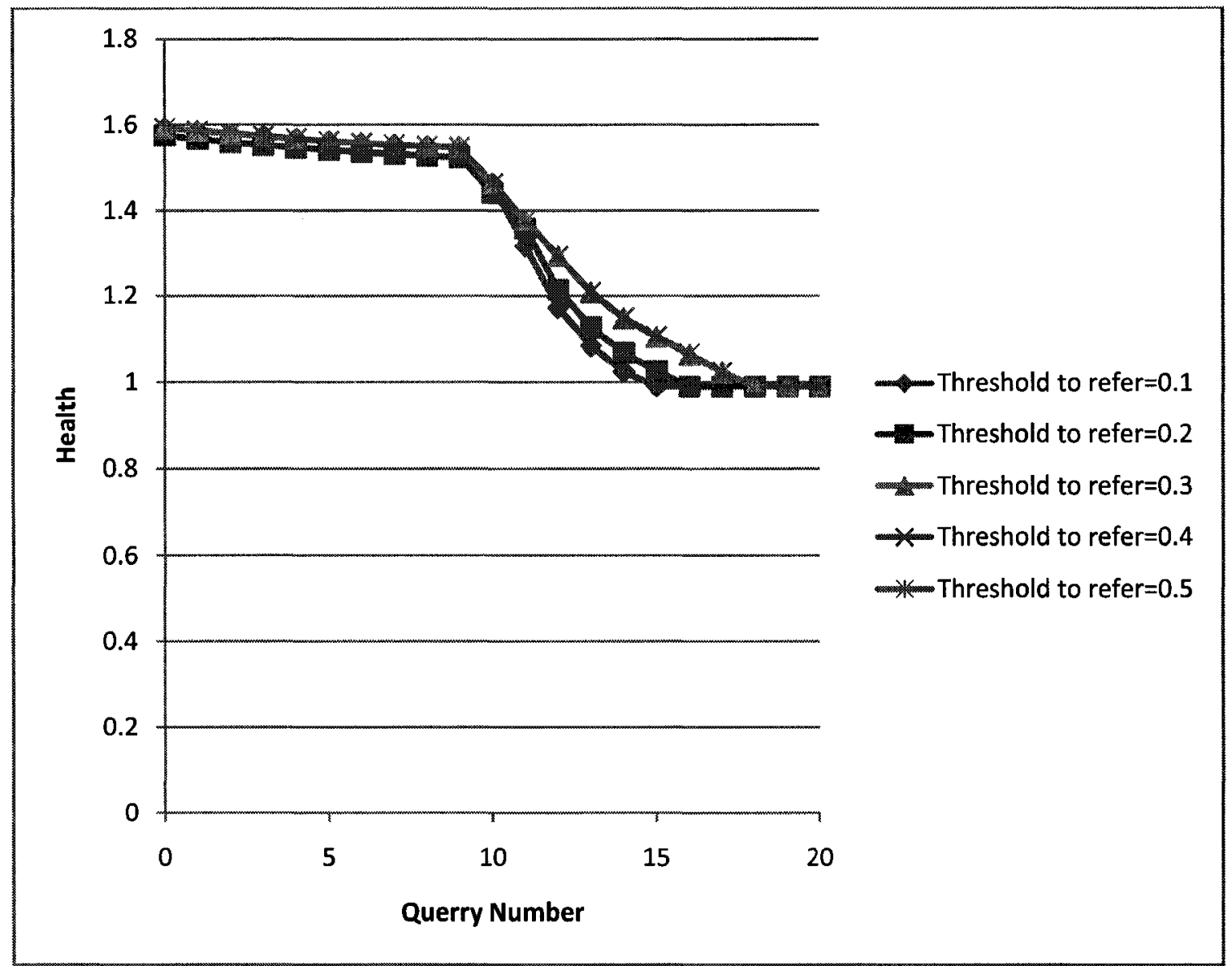

FIGURE 21: Effect of Learning on Health ( $\alpha=0.15)$

CONCLUSION. Lower thresholds to refer facilitate faster learning as it promotes finding experts in the system more quickly.

\subsection{Trust}

This section examines the setup and results of evaluating concept knowledge at a local level using trust automata functions. This simulation contains 400 agents, with 39 experts in one domain, 360 consumer agents asking about a related domain and one oracle. Two new features are introduced in this simulation: 
1. Agents used the three trust functions (linear, exponential, and sigmoid) in trust automata with 5, 10 and 15 states to ascertain the effect of increasing the number of automaton states.

2. An initially malicious/useless oracle (named oracle A) provides a series of 7 pieces of useless pieces of concept knowledge to the agents, followed by 1 useful concept relation. Using 7 pieces of useless concept knowledge in combination with automata of 5,10 and 15 states, will exercise different portions of the trust function curves and demonstrate the utility of building distrust slowly.

Figure 22 illustrates the series of concept relations given by the oracle agent. The first seven (in red) are all useless to the consumer agents in the network, the final concept (in green) relation provides utility; i.e., it provides a relation such that adapting interests to the related concept will lead to learning in the domain agents need to learn about.

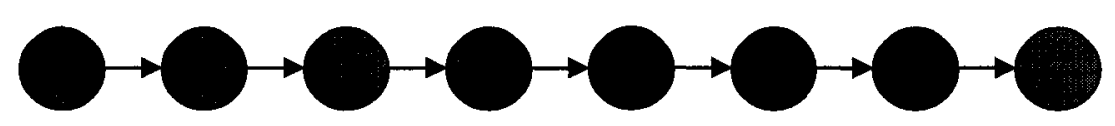

FIGURE 22: Chain of concept relations from Oracle

An analogy of this simulation would be searching online, with the help of a friend say, on MSN. We start with an initial query and find nothing, our friend then messages a suggestion for a new search query, we use that and still nothing, so the friend gives another suggestion; this continues for some amount of time. Naturally 
we do not give up immediately, but usually over time we tend to start ignoring suggestions from our friend if they provide little use. In the end we may sign-off MSN completely and block the friend or alternately we may persist until he/she gives a good suggestion. The oracle in this simulation is akin to the friend in the example.

This simulation is intended to:

1. Demonstrate the effectiveness of the various trust functions and trust automata states.

2. Prove agents can learn in spite of "noisy" concept knowledge.

The results for effectiveness (quality), efficiency (accuracy) and authoritativeness of these simulations are presented below.

\subsubsection{Effectiveness/Quality Results}

The performance of the three trust functions is evaluated by varying the number of states in each of the trust automata. The $\mathrm{n}^{\text {th }}$ best quality metric is shown below. Quality remains almost constant (with small jumps as agents initially try new concepts) until a concept is found that provides benefit and useful experts can be found in the network. Since initially bad, (useless or malicious) concept knowledge is initially propagated in the network, it would be expected that trust automata with fewer states would build distrust faster and thus would ignore late-coming concept knowledge which would be useful to the agent. Experimentally, this expectation is 
validated, as a larger number of automaton states results in a better performance across all trust functions as shown in Figure 23. A greater number of automaton states works to build distrust more slowly and facilitates the acceptance of good concept knowledge at a later stage in the simulation.

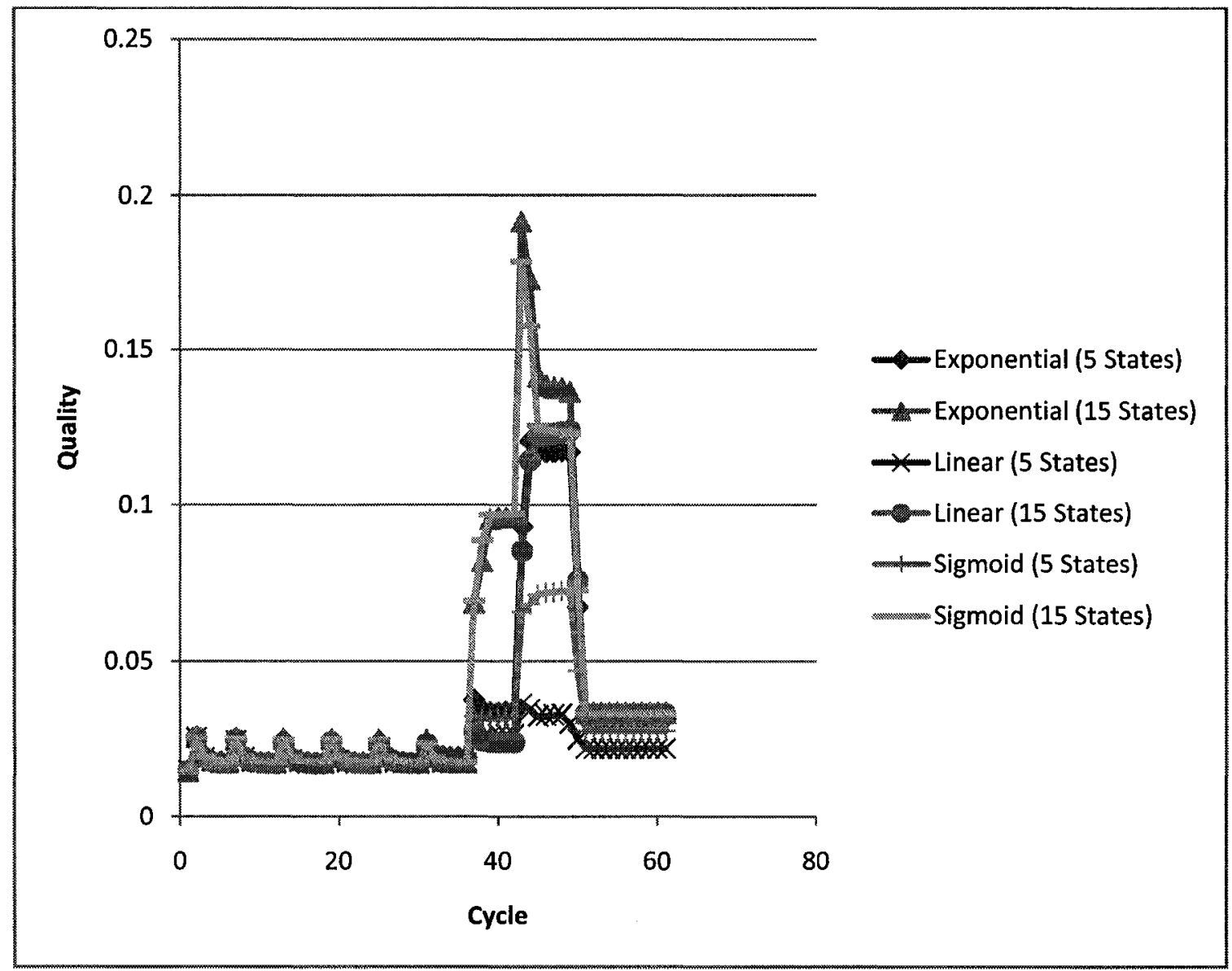

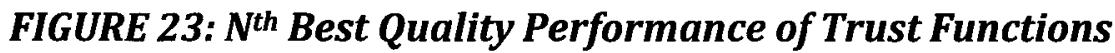

The number of states in the automaton is not the only variable affecting the performance of quality. The nature of the trust function curve itself affects the probability with which concept knowledge will be accepted. In Figure 24, the exponential curve outperforms its counterparts when the number of states is 
controlled. This would suggest that building trust slowly may initially use more useless concept knowledge, but in the end may pay higher dividends. The particularly bad performance of the linear trust function is due to the nature of the linear function itself. A linear function builds distrust initially much faster than the other curves and this tends to cause agents to ignore concepts that may at a later time help them or oracles which may at a later time help them.

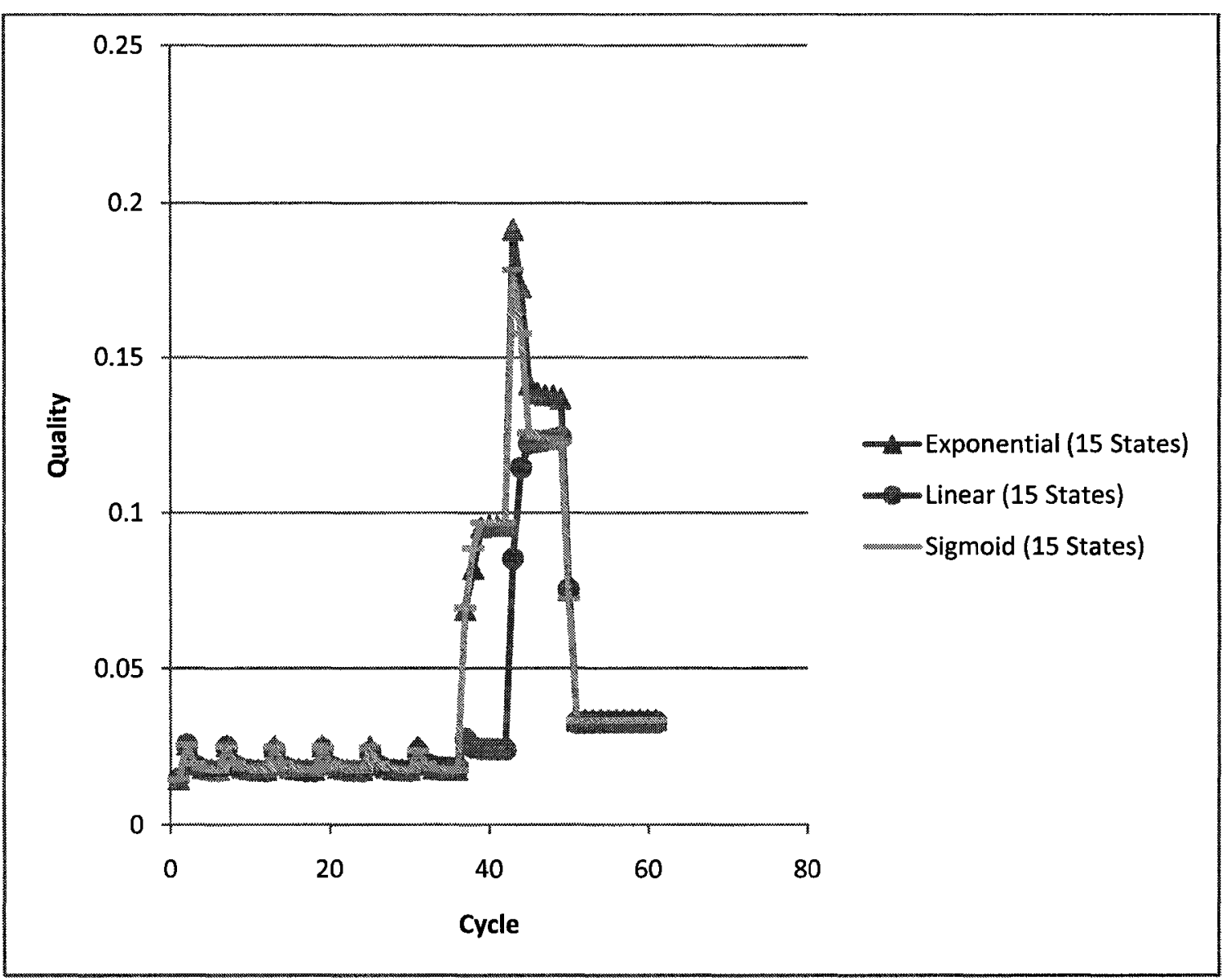

FIGURE 24: Effect of trust function curve

The exponential trust function with 10 states is notable in that it outperforms the exponential trust function with 15 states initially, tends to peak higher and then 
drops below, as can be seen in Figure 25. This is due to the more restrictive nature of the curve with fewer states. With fewer states a smaller number of increases in distrust will result in a higher probability that concept knowledge will not be used. In this simulation this restrictiveness tended to favour early usage of the good piece of concept knowledge, and reject concept knowledge received earlier, thus the reason for the earlier climb in quality. Ultimately this earlier use of concept knowledge also facilitated the higher peak in quality as more agents used the good piece of concept knowledge earlier. However, the exponential trust function with 15 states outperforms the one with 10 after the peak, as more agents gradually use the good piece of concept knowledge.

The peak quality achieved by the exponential automaton with 10 states is 0.204 . In section 8.1.1 the peak quality achieved in the baseline referral network (without concept knowledge) was 0.215 . This demonstrates that a referral network in spite of malicious or useless concept knowledge can ultimately achieve network quality that rivals a traditional network. Again it should be noted that we do not distinguish between malicious agents or uninformed oracles. This represents a significant strength of the research reported here. 


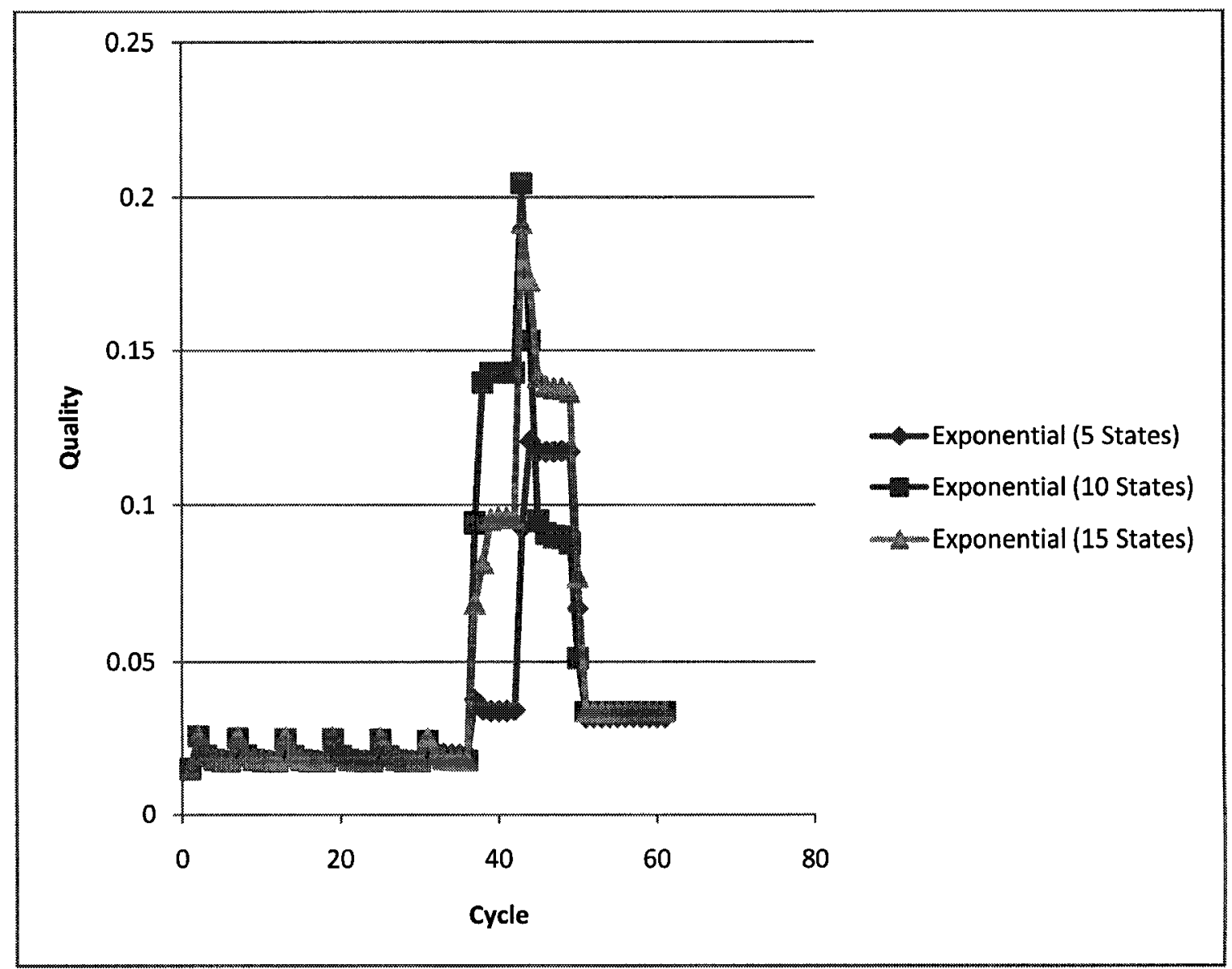

FIGURE 25: Exponential Trust Function

CONCLUSION. The interplay of both the nature of the trust function curve and the number of states affects the propagation of concept knowledge and thus the quality of the network. Generally non-linear, slow rising trust functions with a larger number of states will provide better quality results.

\subsubsection{Efficiency/Accuracy}

As with quality, the accuracy of referral networks with trust automata utilizing slower rising trust functions and more states tend to outperform those which have faster rising trust functions with fewer states. Again, good concept knowledge is 
better utilized at a later stage in the simulation if agents on the average tend to trust each other more, resulting in higher accuracy measures.

Figure 26 shows results of the accuracy ratings for all three trust fucntions with 5 and 15 states. Trust automata with 10 states are left off for the sake of clarity, but performed moderately better than the automata with 5 states and were inferior to the ones with 15 states, for all trust functions.

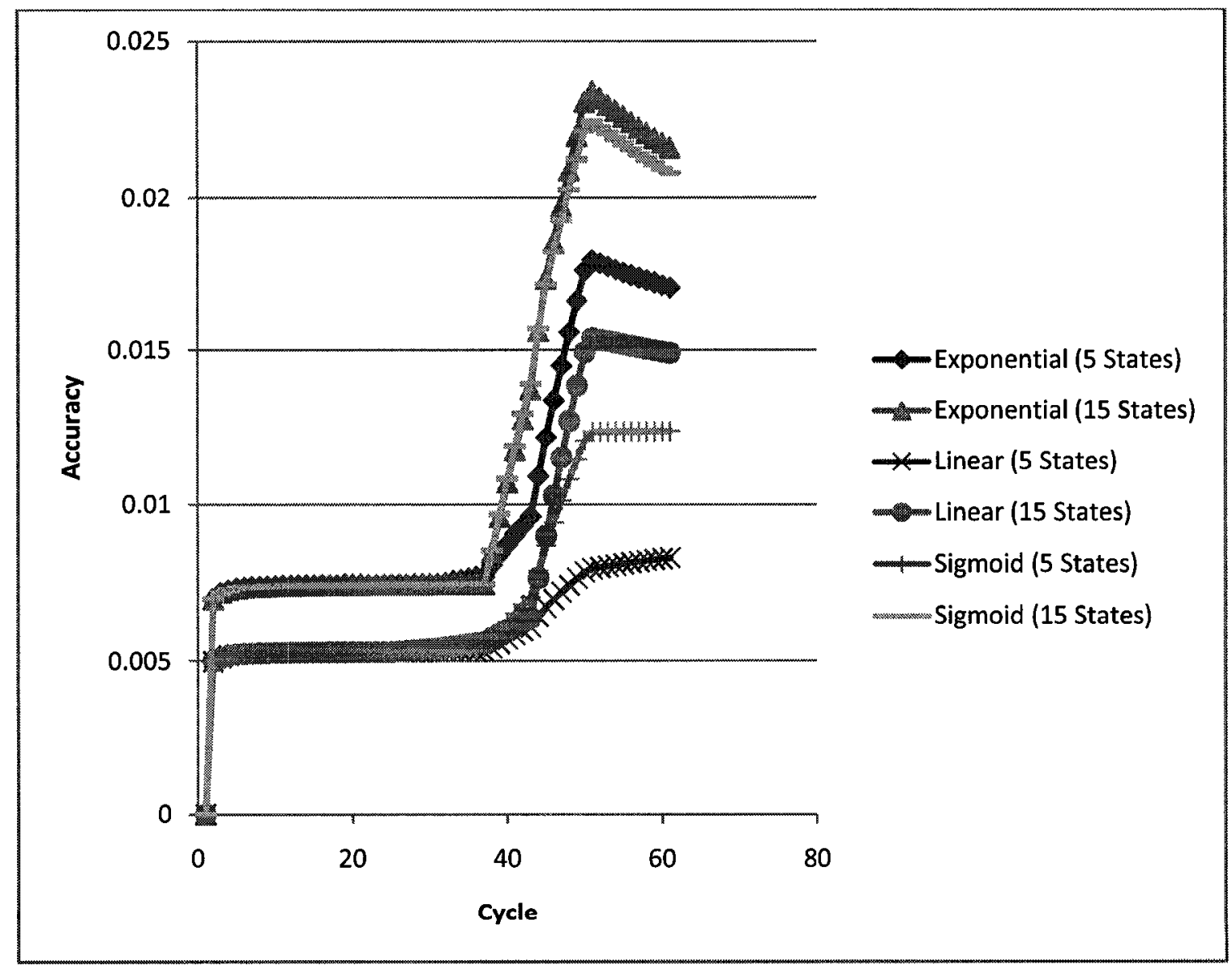

FIGURE 26: Accuracy Performance of Trust Functions 
CONCLUSION. As with quality a slow-rising trust function with a larger number of states allows the better usage of concept knowledge and facilitates higher accuracy.

\subsubsection{Oracle Trust and Agent Health}

There is no distinction between an oracle making a valid suggestion for which no experts can be found and an oracle which gives invalid suggestions (i.e., two domains that are not related). Thus, in this simulation there is no distinction between labelling oracle $A$ as useless or malicious; unless content can be found the oracle's suggestions will still be discarded and trust will be decreased in the oracle. Health ratings motivate distrust (or trust) in an oracle (or agent sharing concept relations) and trust affects how many concepts are used or discarded. To show this we examine five facets of agents which interact with the oracle; the number of concepts it received from the oracle, how many were used, the distrust rating in the oracle, initial and final health of the neighbouring agent. Results for the exponential trust function with 5 states are shown in Table 3.

\begin{tabular}{|l|l|l|l|l|l|}
\hline Neighbour & $\begin{array}{l}\text { Concepts } \\
\text { Received }\end{array}$ & $\begin{array}{l}\text { Concepts } \\
\text { Used }\end{array}$ & $\begin{array}{l}\text { Distrust } \\
\text { Rating }\end{array}$ & $\begin{array}{l}\text { Initial } \\
\text { Health }\end{array}$ & $\begin{array}{l}\text { Final } \\
\text { Health }\end{array}$ \\
\hline 7 & 8 & 5 & 1.0 & 1.63 & 1.63 \\
\hline 67 & 8 & 5 & 1.0 & 1.63 & 1.63 \\
\hline 78 & 8 & 5 & 1.0 & 1.63 & 1.63 \\
\hline 165 & 3 & 2 & 0.0183 & 1.63 & 1.63 \\
\hline 197 & 8 & 5 & 1.0 & 1.63 & 1.63 \\
\hline 287 & 6 & 3 & 0.135 & 1.63 & 1.63 \\
\hline 302 & 8 & 5 & 1.0 & 1.63 & 1.63 \\
\hline 310 & 6 & 5 & 1.0 & 1.63 & 1.63 \\
\hline 371 & 6 & 5 & 1.0 & 1.63 & 1.63 \\
\hline
\end{tabular}

TABLE 3: Health and Trust for Exponential Function (5 States) 
As can be seen by the results in Table 3, an initially useless oracle is penalized too quickly in an automaton with few states. Agents build distrust too quickly and never utilize the useful piece of concept knowledge; they distrust the oracle too much and thus ignore the useful suggestion from the oracle that arrives later in the simulation. Results for the exponential trust function with 15 states show a different picture. The oracle is not quickly penalized in terms of distrust, and all but two agents are able to try all the concepts suggested and eventually utilize the useful piece of concept knowledge as reflected by their final health.

\begin{tabular}{|l|l|l|l|l|l|}
\hline Neighbour & $\begin{array}{l}\text { Concepts } \\
\text { Sent }\end{array}$ & $\begin{array}{l}\text { Concepts } \\
\text { Used }\end{array}$ & $\begin{array}{l}\text { Trust } \\
\text { Rating }\end{array}$ & $\begin{array}{l}\text { Initial } \\
\text { Health }\end{array}$ & $\begin{array}{l}\text { Final } \\
\text { Health }\end{array}$ \\
\hline 7 & 8 & 8 & 0.0695 & 1.63 & 0.99 \\
\hline 67 & 8 & 8 & 0.0695 & 1.63 & 0.99 \\
\hline 78 & 8 & 8 & 0.0695 & 1.63 & 0.99 \\
\hline 165 & 8 & 8 & 0.0695 & 1.63 & 0.99 \\
\hline 197 & 6 & 5 & 0.0357 & 1.63 & 1.63 \\
\hline 287 & 6 & 5 & 0.0357 & 1.63 & 1.63 \\
\hline 302 & 8 & 8 & 0.0695 & 1.63 & 0.99 \\
\hline 310 & 8 & 8 & 0.0695 & 1.63 & 0.99 \\
\hline 371 & 8 & 8 & 0.0695 & 1.63 & 0.99 \\
\hline
\end{tabular}

TABLE 4: Health and Trust for Exponential Function (15 States)

Tables 3 and 4, do not paint a complete picture by themselves as they only show local interactions between the oracle and it's neighbours. Examining Table 3 may seem to indicate that no learning in the correct domain was done at all with the 5 state exponential trust automaton; however, this is not true. Table 5 presents the global averages of initial and final health of the agents in the network for all trust 
functions and automata states. As can be seen in table 5, some consumer agents for lower automaton states do learn about the correct domain as the average final health is lower than average initial health. This is due to the fact that concept knowledge is propagated from consumer to consumer (who act as oracle when they contain concept relations), not just from the oracle. Thus distrust is distributed across the neighbours of an agent, and allows the eventual acceptance of the useful piece of concept knowledge (it may come from an agent that has only suggested 1 piece of bad concept knowledge). Note that in Table 5 the best health an agent can achieve is 0.99 , as they never learn about their initial domain of interest.

\begin{tabular}{|l|l|l|l|}
\hline Trust Function & Number of States & Initial Health & Final Health \\
\hline Exponential & 5 & 1.4852 & 1.1144 \\
\hline Exponential & 10 & 1.4852 & 0.99 \\
\hline Exponential & 15 & 1.4852 & 0.99 \\
\hline Linear & 5 & 1.4852 & 1.4008 \\
\hline Linear & 10 & 1.4852 & 1.1922 \\
\hline Linear & 15 & 1.4852 & 1.1465 \\
\hline Sigmoid & 5 & 1.4852 & 1.2516 \\
\hline Sigmoid & 10 & 1.4852 & 1.0772 \\
\hline Sigmoid & 15 & 1.4852 & 0.99 \\
\hline
\end{tabular}

TABLE 5: Global Average Health Ratings

CONCLUSION. Examining the interconnectedness of health and trust affirms the need for distrust to be built slowly. It allows agents a level of resilience against useless concept relations and promotes persistence that can lead to beneficial results. 


\subsection{Transitivity}

It is nearly impossible to learn about one thing in isolation. Take, for instance, the real-world situation of learning about Einstein's general theory of relativity. It is nearly impossible to learn about Einstein's general theory of relativity without becoming aware of space-time curvature, which in turn introduces black holes, event horizons, worm-holes and so on. Thus learning about the general theory of relativity leads to transitively learning about other related domains. The objective of this simulation is to demonstrate agents can learn transitive concept relations.

This simulation again splits the experts into two groups one with expertise in domain 1 and the other with expertise in domain 2. One oracle contains two pieces of transitive concept knowledge; the first relates domain 0 to 1 (concept A), the second relates 1 to 2 (concept B). Consumer agents are initially interested in domain 0 but would benefit from learning from experts in domain 1 and 2 (related domains). Thus they benefit initially from utilizing the concept relation $0 \rightarrow 1$. As a consequence of showing interest in domain 1 agents are then provided the concept relation $1 \rightarrow 2$ from the oracle, which in turn provides utility to the consumer agents.

\subsubsection{Health Results}

The health metric best demonstrates the effect of learning transitive concept relations, as it shows implicitly several features: 
1. Concepts are propagated from the oracle to the consumer agents. If related concepts were not propagated the health of agents would never improve as they would never learn about needed domains.

2. Experts are found for both domains. Without finding experts agents could never learn and thus their health would suffer.

Figure 27 depicts the average health and the variance in the health metrics of agents after each query. Consumer agents begin using concept $A$ at query 10 (shown in the graph with an arrow), hence the small plateau at the start of the curve. The descending curve to query number 30 denotes the time when agents are learning about domain 1 . Also during this time the oracle provides concept $B(1 \rightarrow 2)$. The second plateau from cycle 30 to 70 indicates that agents have completely learned about domain 1 using concept A. Once their health has stagnated for enough time, consumer agents then begin using concept $B$ at cycle 71 (indicated by the second arrow) and continue to learn about domain 2 until cycle 91, where they exhibit perfect health. 


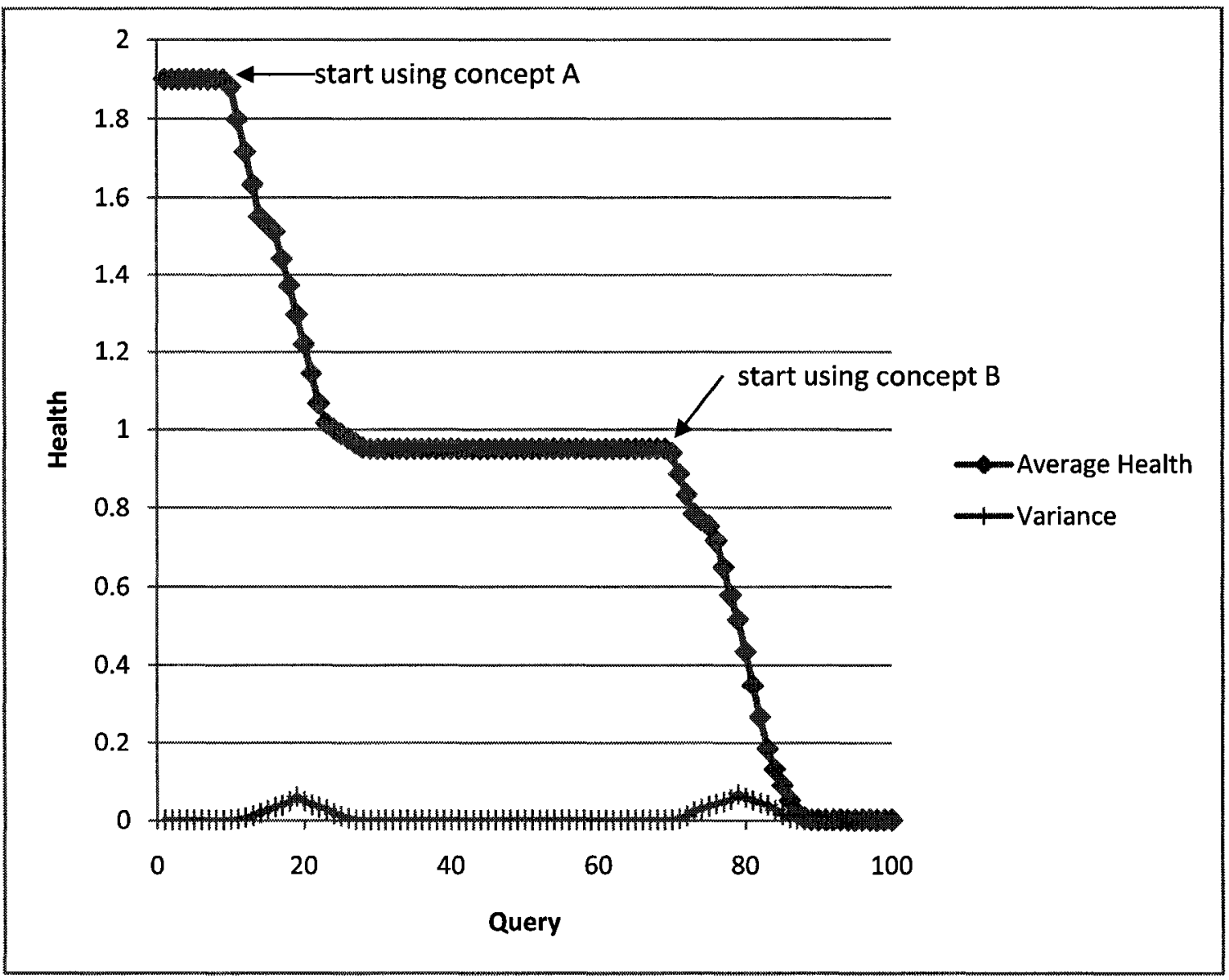

FIGURE 27: Average Health and Variance

Some agents may find experts quicker than others and begin learning earlier than others and the variance of the average health increases. Once all consumer agents have learned the concept the variance settles to zero. This is shown for concept A, where agents start learning about domain 1 at cycle 10; some learn earlier and thus the variance in health increases, but then begins to subside at cycle 20 as all agents learn domain 1 . This is shown for concept $B$ as well at cycle 71 , where variance increases and then begins to subside around cycle 81 , and eventually settles to 0 at cycle 92. 


\subsubsection{Quality}

Since learning is also employed in this simulation, the quality results are similar to those presented in section 8.2, and are left out for the sake of brevity. However, a powerful aspect of this transitivity simulation is its ability to demonstrate that a referral network with concept knowledge can rival the quality of a referral network without concept knowledge where the "right set of questions to ask" (interests) is known initially. For example, take two separate referral networks called RF1 and RF2. The first referral network, RF1 contains consumer agents who are interested in domain 1, the second RF2 contains consumer agents interested in domain 2. Both networks have experts in domains 1 and 2. RF1 should produce similar quality measures as when the concept referral network in this simulation (for the sake of simplicity let us name it CRF) is learning concept A. Additionally RF2 should produce similar quality results as the CRF network in this simulation when it is learning concept B. As shown in section 8.1, referral networks with concept knowledge perform equally with normal referral networks that initially were asking the correct questions of experts. Learning transitive concepts is no different as is shown in Table 6 . The direct and $\mathrm{n}^{\text {th }}$ best quality metrics are similar when comparing RF1 to the CRF network (and consumer agents are learning concept A) and when comparing RF2 to the CRF network (and consumer agents are learning from concept B). 


\begin{tabular}{|l|l|l|l|l|}
\hline Measure & RF1 & CRF Concept A & RF2 & CRF Concept B \\
\hline Direct Quality & 0.4311 & 0.4636 & 0.4311 & 0.4201 \\
\hline N $^{\text {th }}$ Best Quality & 0.2152 & 0.2315 & 0.2152 & 0.2049 \\
\hline
\end{tabular}

TABLE 6: Comparison of Quality

\subsubsection{Accuracy and Authoritativeness}

Similar results are found for this simulation as those found in section 8.2 for referral networks with concept knowledge and are left out for the sake of brevity.

\subsubsection{Concept Propagation}

As shown in section 8.1, concept A propagates quickly through the network. As shown in Table 7 , concept propagation then halts from query number 3 to 10 ; agents are at this time deciding if they need to use a concept (if their health has stagnated) and as they adapt their interests to domain 1 they receive the concept relation $1 \rightarrow 2$ from the oracle, which is quickly propagated through the network by query number 13 . The 20 remaining agents with neither concept A nor concept B are the 20 experts in domain 2, as they never show interest in a domain for which there is a concept relation. 


\begin{tabular}{|l|l|l|l|}
\hline $\begin{array}{l}\text { Query } \\
\text { Number }\end{array}$ & $\begin{array}{l}\text { Concepts } \\
\text { Shared }\end{array}$ & $\begin{array}{l}\text { Agents without } \\
\text { concept A }\end{array}$ & $\begin{array}{l}\text { Agents without } \\
\text { concept B }\end{array}$ \\
\hline 1 & 72 & 331 & 399 \\
\hline 2 & 914 & 52 & 399 \\
\hline 3 & 2789 & 40 & 399 \\
\hline 4 & 2910 & 40 & 399 \\
\hline 5 & 2910 & 40 & 399 \\
\hline 6 & 2910 & 40 & 399 \\
\hline 7 & 2910 & 40 & 399 \\
\hline 8 & 2910 & 40 & 399 \\
\hline 9 & 2910 & 40 & 399 \\
\hline 10 & 2910 & 40 & 399 \\
\hline 11 & 3055 & 29 & 335 \\
\hline 12 & 4255 & 20 & 52 \\
\hline 13 & 6349 & 20 & 20 \\
\hline
\end{tabular}

TABLE 7: Concept Propagation

After receiving both pieces of concept knowledge the consumer agents then set about to find experts in domain 1 and subsequently domain 2 . At this point the oracle becomes completely isolated from the network, as it is providing neither answers nor referrals in domains the consumers are interested in. If the oracle contained a further piece of transitive knowledge, say the concept relation $2 \rightarrow 3$, this concept would have never propagated to the consumer agents (as it is completely isolated when consumers are showing interest in domain 2). This simulation supports the conjecture made in section 6.4 that concept knowledge should reside in the experts themselves. In this way no matter how much convergence on experts occurred, the agents would still learn needed concept relations. So the concept relation $0 \rightarrow 1$ would reside with experts in domain 0 , and the concept relation $1 \rightarrow 2$ would reside with experts in domain 1 . As agents found experts for domain 0 they would learn about the concept relation $0 \rightarrow 1$, they would 
then seek out experts for domain 1 , where they would learn the concept relation 1 $\rightarrow 2$, etc. Another solution would be to modify the neighbor selection to maintain connections to useful oracles. Policies to ensure this are considered future work and described in chapter 9.

CONCLUSION. The ability for agents to learn transitively related concepts from a single oracle demonstrates that an oracle can act as a guide to learning. Through successive suggestions an oracle can lead agents to learn about a series of related domains. The need for the oracle to maintain connectivity in the network is also emphasized.

\subsection{Context}

This simulation splits the experts into two groups each with expertise in a different domain. Consumer agents are also split into two groups, the first called class $A$ with initial interest in domain 0 but for which searching domain 1 will provide useful answers, the second called class B with initial interest in domain 1 but for which searching in domain 2 will provide useful results. Thus class A agents will find the concept suggestion of $0 \rightarrow 1$ useful while class $B$ agents will find $0 \rightarrow 2$ useful. Two oracles are also present in the network each with concept knowledge relating the same domain (0) to different domains (1 and 2). Thus one oracle is considered a class A oracle, providing relevant concept knowledge to class A consumer agents, and the other is a class B oracle that provides relevant concept knowledge to class B consumer agents. 
This simulation is meant to model, for example, carpenter and math agents asking about the concept "table" and the oracles providing two suggestions; one relating table to "furniture" and the other to "matrices". It is the agent's job to distinguish the utility of the concept knowledge provided based on their own internalized needs. Thus this simulation has two objectives:

1. Demonstrate the ability of concept knowledge to distinguish context within or outside of a domain.

2. Reinforce the need for a feedback mechanism to determine the utility of concept knowledge.

\subsubsection{Health Results}

The purpose of this simulation is to show that agents can discriminate between two concepts based on what is useful to them. To this end, Figure 28 plots the average health and the variance in the health metrics of the two classes ( $A$ and $B$ ) of consumer agents over time. As each class of agent makes use of the correct concept relation and learns in the domain which is useful to them, the health of agents moves to zero (perfect health). 


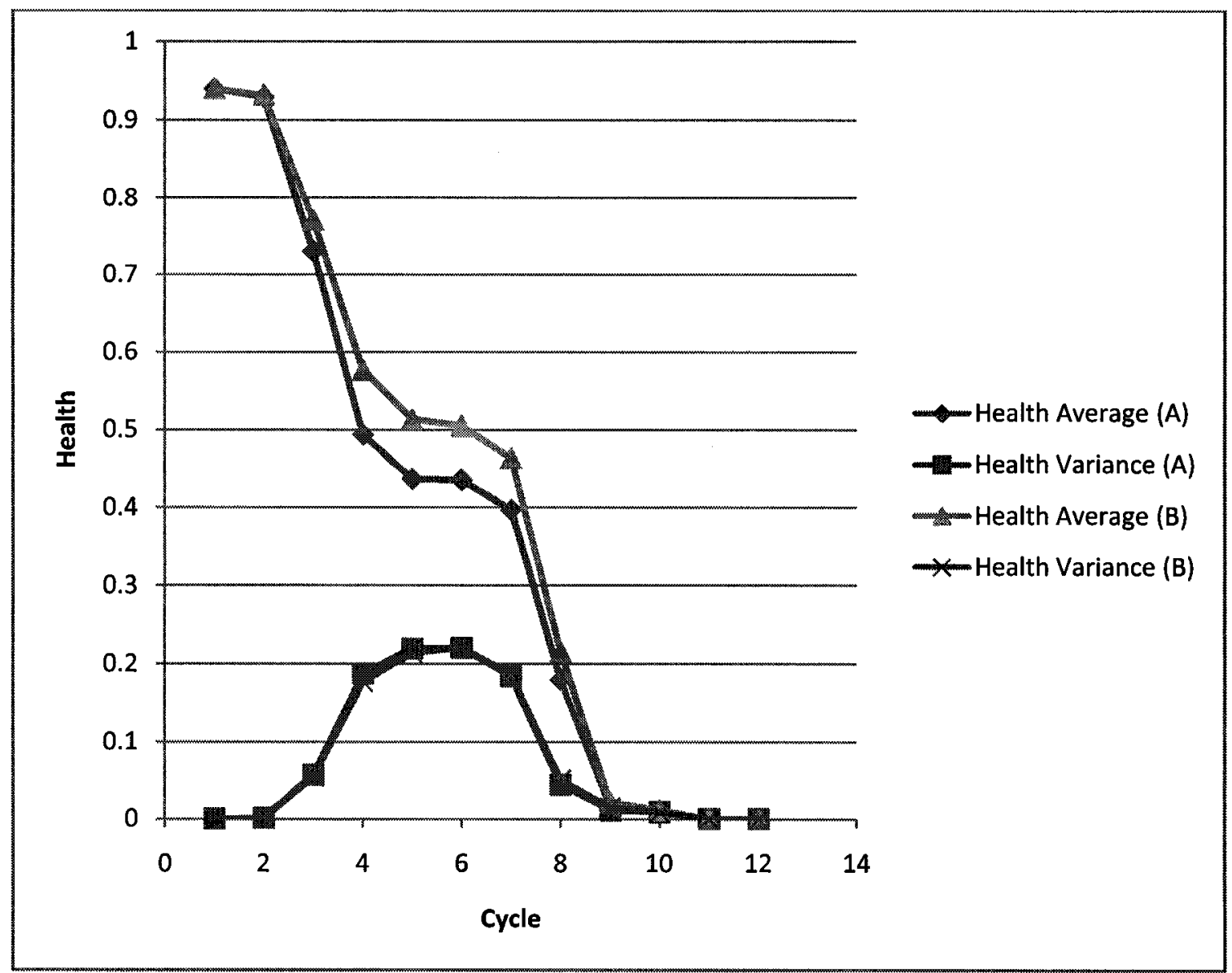

FIGURE 28: Health over time

The variance "hump" indicates that this learning is not done all at the same time. Some agents may initially get the correct concept relation, while others do not. Variance in the average health measure for each class of consumer agent will increase as some agents in their class learn and others do not, and then decrease as all agents in the class eventually learn the related domain that is useful to them.

As agents learn which concept is useful to them, they reflect this utility in their trust ratings of the oracle (or agent) who gave them this knowledge. To demonstrate this fact the trust ratings of a class $A$ oracle are presented in Table 8 , over the entire 
simulation lifecycle. The agents on the left hand column represent neighbouring agents that the oracle shared its concept relation with. The number 1 through 12 along the top denote the simulation cycle, the entries in the body of the table denote the trust rating of the agent in the oracle.

\begin{tabular}{|l|l|l|l|l|l|l|l|l|l|l|l|l|}
\hline Agent & \multicolumn{10}{|c|}{ Simulation Cycle } \\
\hline & $\mathbf{1}$ & $\mathbf{2}$ & $\mathbf{3}$ & $\mathbf{4}$ & $\mathbf{5}$ & $\mathbf{6}$ & $\mathbf{7}$ & $\mathbf{8}$ & $\mathbf{9}$ & $\mathbf{1 0}$ & $\mathbf{1 1}$ & $\mathbf{1 2}$ \\
\hline $\mathbf{1 9}$ & 0 & 0 & 0 & 0 & 0 & $\mathbf{0 . 0 1 8}$ & $\mathbf{0 . 0 1 8}$ & $\mathbf{0 . 0 1 8}$ & $\mathbf{0 . 0 1 8}$ & $\mathbf{0 . 0 1 8}$ & $\mathbf{0 . 0 1 8}$ & $\mathbf{0 . 0 1 8}$ \\
\hline $\mathbf{9 5}$ & 0 & 0 & 0 & 0 & 0 & 0 & 0 & 0 & 0 & 0 & 0 & 0 \\
\hline $\mathbf{1 4 6}$ & 0 & 0 & 0 & 0 & 0 & 0 & 0 & 0 & 0 & 0 & 0 & 0 \\
\hline $\mathbf{2 2 9}$ & 0 & 0 & 0 & 0 & 0 & $\mathbf{0 . 0 1 8}$ & $\mathbf{0 . 0 1 8}$ & $\mathbf{0 . 0 1 8}$ & $\mathbf{0 . 0 1 8}$ & $\mathbf{0 . 0 1 8}$ & $\mathbf{0 . 0 1 8}$ & $\mathbf{0 . 0 1 8}$ \\
\hline $\mathbf{2 8 3}$ & 0 & 0 & 0 & 0 & 0 & 0 & 0 & 0 & 0 & 0 & 0 & 0 \\
\hline $\mathbf{3 5 3}$ & 0 & 0 & 0 & 0 & 0 & 0 & 0 & 0 & 0 & 0 & 0 & 0 \\
\hline $\mathbf{3 7 2}$ & 0 & 0 & 0 & 0 & 0 & $\mathbf{0 . 0 1 8}$ & $\mathbf{0 . 0 1 8}$ & $\mathbf{0 . 0 1 8}$ & $\mathbf{0 . 0 1 8}$ & $\mathbf{0 . 0 1 8}$ & $\mathbf{0 . 0 1 8}$ & $\mathbf{0 . 0 1 8}$ \\
\hline $\mathbf{3 7 9}$ & 0 & 0 & 0 & 0 & 0 & $\mathbf{0 . 0 1 8}$ & $\mathbf{0 . 0 1 8}$ & $\mathbf{0 . 0 1 8}$ & $\mathbf{0 . 0 1 8}$ & $\mathbf{0 . 0 1 8}$ & $\mathbf{0 . 0 1 8}$ & $\mathbf{0 . 0 1 8}$ \\
\hline
\end{tabular}

TABLE 8: Trust ratings of the class A Oracle

As can be inferred from Table 8, agents 19, 146, 372 and 379 are class B consumer agents, and thus after trying the concept relation $0 \rightarrow 1$ from the oracle increased their distrust in the oracle, as it did not provide them with useful results. Agents 95, 146,283 , and 352 are class A consumer agents and find the concept relation $0 \rightarrow 1$ useful and thus do not penalize the oracle. Similar results were obtained for the class B oracle and are left out for the sake of brevity.

CONCLUSION. The ability for agents to locally and subjectively evaluate suggestions allows them to distinguish useful concepts from useless ones and additionally allows them to establish useful oracles within the network. As agents can determine 
useful context, meaning becomes an emergent property; agents will utilize concept relations which hold the most applicable meaning for their internalized needs.

\subsection{Summary}

Several key points are shown in this chapter. Firstly, the utility of concept knowledge in finding new sources of knowledge while maintaining key metrics of referral networks is demonstrated. Secondly, the effects of learning are shown; if agents do not learn to the extent of the best experts in the network, slower learning rates are desired. Thirdly, the utility of using non-linear, slow rising trust automaton with a larger number of states is shown to allow agents persistence and a greater degree of resilience in the face of noisy concept knowledge. Fourthly, transitive concept relations are shown as an effective learning tool for agents, while the importance of connectivity to the oracle is highlighted. Finally, the ability for agents to distinguish context and consequently determine meaning based on their own internalized needs is shown. Having presented the usefulness of concept knowledge and evaluating its utility based on local evaluation, the next chapter provides concluding remarks and future directions for work in this field. 


\section{Chapter 9}

\section{Conclusions and Future Work}

\subsection{Introduction}

This chapter reaffirms the stated contributions of this thesis, whilst presenting some future directions for work in this field.

\subsection{Conclusion}

There were three main goals for this thesis:

1. To substantiate the usefulness of concept knowledge and adapting interests to find new sources of knowledge.

2. Introduce and show the validity of a learning evaluation feedback mechanism.

3. Show the need for localized trust evaluation based on utility.

The notion of relational concept knowledge was introduced and shown to effectively allow agents to find alternate sources of concept knowledge in the network. This is meant to model humans' innate ability to use relational concept knowledge; for instance, when searching online and a certain concept does not provide useful results we then adapt our search based on related concepts. Agents in a referral network are very well adapted to find experts and give referrals; the addition of 
concept knowledge allows them to leverage this innate ability to learn new and related concepts.

Health was introduced as a mechanism to "guide" agents in their learning. Just as humans can evaluate the usefulness of a related concept via its application to solve a problem, agents must have a method to evaluate the usefulness of learning. This thesis provides a simplified mechanism where an evaluation blueprint is given to the agent; it does not attempt to introduce a mechanism for agents to determine internally if learning a related domain is useful. Further work on meta-data ontologies or concept maps may help an agent reason intelligently about useful concepts, just as humans do, but until then agents must be guided by an outside source.

Trust is very important in any sort of social interaction. The local evaluation of concept knowledge based on an internal feedback mechanism allows agents to determine the utility of shared concepts. This utility used as trust allows agents to determine useful providers of concept knowledge based on their needs. It acts to prevent malicious or useless concept knowledge providers from continually misguiding consumer agents in the network.

\subsection{Applicable Domains}

Online meta-search engines such as InfraSearch provide a very promising framework for research reported in this thesis. Meta-search engines realize that 
information is distributed across a network and may never be successfully synthesized and centralized on one single server or cluster of servers. This research proposes that domain-based communities of experts form and provide three essential ingredients to search:

1. Expertise which leads to answers,

2. Concept relations which results in suggestions,

3. Links to agents in related domains or sub-domains, which facilitates concept related referrals.

Search is then done by a meta-search server which aggregates results from experts in different domains (the equivalent of information providers in InfraSearch). Answers can be provided as well as concept relations and referrals to experts in related domains. An example InfraSearch network is shown in Figure 29.

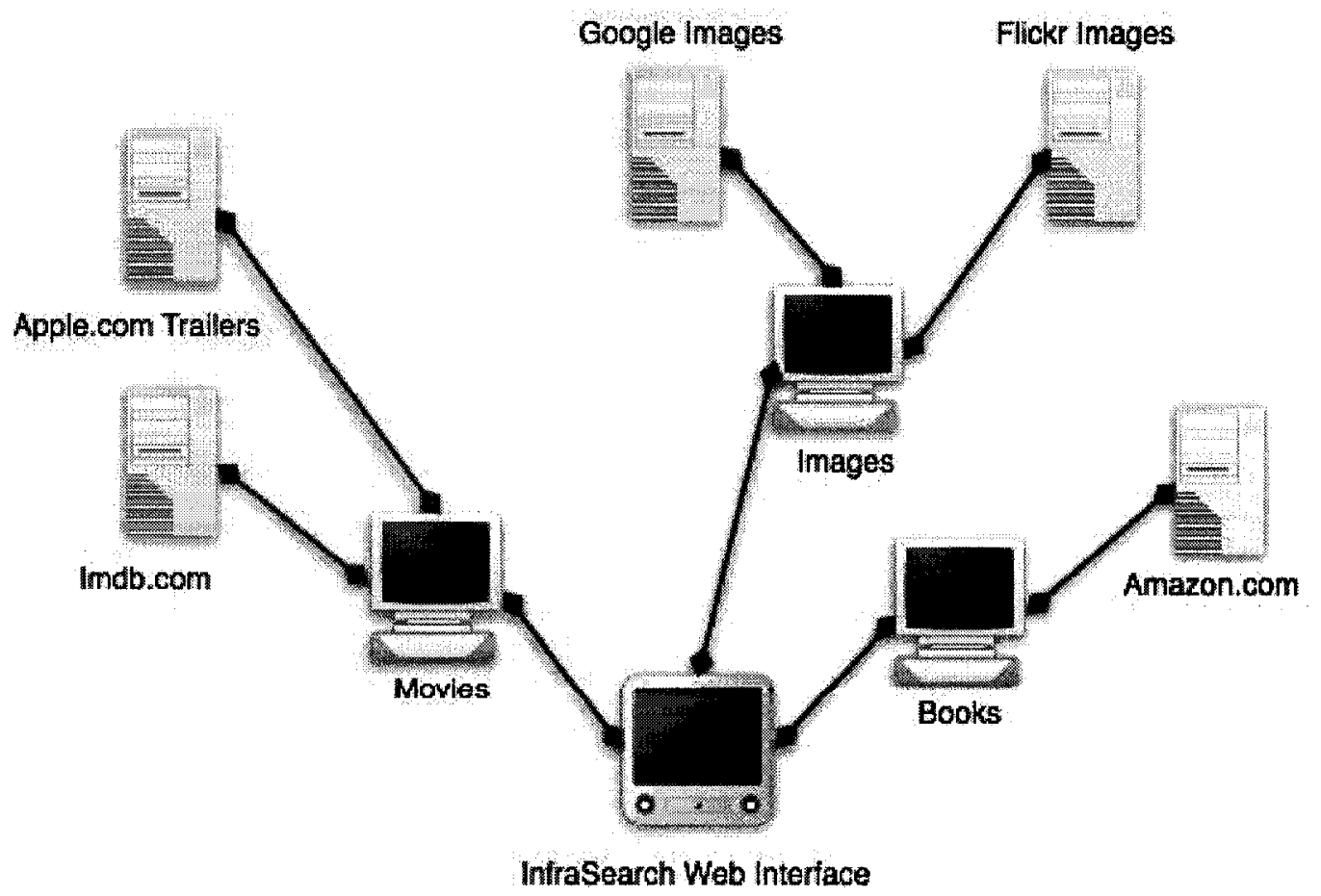

FIGURE 29: InfraSearch Overview 
Referral networks are also useful recommender systems as they allow agents to "know who provides ratings to them" [38] instead of traditional collaborative filtering mechanism which do not reveal the sources of recommendations. Additionally the ability to give conceptual suggestions allows agents to move across communities and thus promotes the notion of discovery, as opposed to finding just similar results based on static interests.

The utility of suggestions in searching is substantial, effectively allowing meaning to become an emergent phenomenon. Agents acting on behalf of a principal (human user) could use suggestions not only from the user, but also from other agents in the system to find related concepts and thus enhance search results. Thus search would employ the power of the community not only to find expertise but new ways to formulate questions.

\subsection{Discussion and Future Direction}

This work provides but a small stepping stone for future work in this field. This section examines some possible future directions for work in this area.

The notion of oracles is really in its infancy. Future oracles will no doubt utilize strong computational constructs to provide better relational concept knowledge. Imagine domain-specific oracles that use concept maps or ontologies to make suggestions or even further synthesize ontologies to provide cross-domain 
suggestions. This work makes no contributions to the mechanism by which suggestions are formulated, thus this field of research lies wide-open. Further work in technologies relating to the Semantic Web may provide useful insights and solutions to the formulation of semantic constructs to replace the concept relations as introduced in this thesis.

Currently agents relay concept knowledge indiscriminately which can lead to noisy concept knowledge; e.g., an agent can give you both useful and useless pieces of concept knowledge. A further contribution would be to examine the utility of only sharing concept knowledge that is useful to the agent itself. This may lead to better trust ratings but may affect the overall referral network quality, as concept knowledge propagation would be reduced. Research into only allowing oracles to disseminate concept knowledge with agents providing referrals to oracles would be another scenario to investigate. However, it is important to note that agents must still be able to function in the presence of noisy and even unreliable concept knowledge.

In this work, concepts are only suggested one at a time. It may be beneficial to ascertain the effectiveness of providing multiple concepts based on a single query. This would facilitate even faster concept propagation, and may serve to reduce the volume of interactions between oracles and agents. 
Interest adaptation is a major point of contribution in this thesis and there exists a rich vein of research in new policies for interest adaptation and interest modelling. Currently interests are progressively adapted by small incremental amounts (at most 0.5 ). New policies that increase interest exponentially or via some function are possible, and may provide superior convergence or higher quality results. Furthermore, the interest model in this thesis assumes agents are only interested in one domain at a particular time. New models of interest may allow an agent to search in multiple domains for experts better suited to providing expertise in more than one domain. This may be an effective tool for better modelling multi-word searching for instance.

As shown in Chapter 6, an agent is not allowed to show more interest in a related domain than in the original domain. This may not always be desirable as related domains may provide more use to an agent than the current. An extension to allow unbounded interest adaptation would be easily implemented and may prove useful to agents.

In section 8.4 .4 , the need for transitive knowledge to be maintained at sources of expertise is shown to be of great importance. However, it may also be important to maintain previously helpful oracles as neighbours. It was not the intention of this thesis to provide a means to do this by altering current methods employed in the neighbour selection policy as backwards compatibility with traditional referral networks was considered important. Relevance of an agent is still chosen based on 
answers and referrals given, not on suggestions. However, beneficial future work would extend the neighbour selection policy to also consider the current health of the agent, previous suggestions supplied by an oracle and the current trust rating of the oracle in making decisions on neighbour selection. For instance, a very simple consumer agent neighbour selection policy might be to re-query an oracle (for any further related concepts) after successfully using one of its suggested concept relations for some time.

Further investigation into better modelling of health stagnation through the interaction of $H_{r}, S_{c}$, and $H_{t}$ simulation parameters would prove useful. It must be ensured that given health has stagnated, enough time is given to the agent to find sources of expertise. Since the interaction of $\mathrm{H}_{r}$ and $\mathrm{H}_{t}$ determine stagnation it must be ensured that they are chosen to realistically represent stagnation of an agent's health. Conceivably, through comprehensive simulation tests guiding design decisions can be given for these two parameters. Alternatively new solutions may replace or augment this mechanism.

\subsection{Summary}

Searching for information has always been a rich field of research and will continue to be as the amount of catalogued information continues to accumulate in digital storage mediums. Leveraging social mechanisms such as referrals is incredibly useful in finding relevant information. However, there is the need to supplement existing social search with semantic constructs that through suggestions promote 
the reformulation of search queries to discriminate context and find new sources of knowledge. Mechanisms to continually evaluate not only answers but suggestions allow the discovery of authoritative experts tailored to subjective needs of the searching agent. In this way computational agents better model how we as humans search for information in the real world. 


\section{Appendix A}

\section{Simulation Configurations}

This appendix describes the simulation variable values for all five simulation groups. All simulations were run on a Dell DIM2400 machine with a Intel Pentium 4 2.4 GHz Celeron CPU, 1.0 GB RAM, with at least $6 \mathrm{~GB}$ free disk space, Windows XP SP2, and Java 6.0 SE.

\section{A.1 Baseline}

\section{Simulation Configuration Parameters}

\begin{tabular}{|l|l|}
\hline Variable & Value(s) \\
\hline NUM_NEIGHBOR_SET_CHANGES & 10 \\
\hline NUM_QUESTIONS_PER_NEIGHBOR_SET & 10 \\
\hline WAITING_LIMIT_FOR_MESSAGES & 1000 \\
\hline MAXIMUM_REFERRAL_CHAIN_LENGTH & 5 \\
\hline MAXIMUM_PERCENTAGE_PERTURBATION_FOR_GENERATING_QUERY & 0.0 \\
\hline MAXIMUM_PERCENTAGE_PERTURBATION_FOR_GENERATING_ANSWER & 0.0 \\
\hline RATE_OF_LEARNING_INTERESTS & 0.5 \\
\hline RATE_OF_LEARNING_FROM_GOOD_ANSWERS $(\alpha)$ & 0.0 \\
\hline ALPHA ( $\alpha$ ') & 0.25 \\
\hline PERCENTAGE_DECAY_FOR_NO_ANSWER & 0.1 \\
\hline BETA ( $\beta)$ & 0.2 \\
\hline W & 0.5 \\
\hline CAPABILITY_THRESHOLD_FOR_EVALUATING_ANSWER & 0.3 \\
\hline CAPABILITY_THRESHOLD_FOR_ANSWERING_QUESTIONER & 0.2 \\
\hline CAPABILITY_THRESHOLD_FOR_REFERING_QUESTIONER_TO_NEIGHBOR & $0.1,0.2,0.3,0.4$, \\
\hline CAPABILITY_THRESHOLD_FOR_ASKING_NEIGHBOR & 0.5 \\
\hline NUM_NEIGHBOURS & 0.01 \\
\hline PERCENT_EXPERTS & 8 \\
\hline
\end{tabular}


Agent Interest and Expertise Setup

\begin{tabular}{|l|l|l|l|}
\hline Agent Type & Interest & Expertise & Target Expertise \\
\hline Experts & $(0.85-1.0,0.01,0.01$, & $\begin{array}{l}(0.5-1.0,0.01,0.01, \\
0.01)\end{array}$ & $(1.0,0.01,0.01,0.01)$ \\
& $0.01)$ & $\begin{array}{l}0.01,0.5-1.0,0.01, \\
(0.01,1.0,0.01,0.01)\end{array}$ \\
\hline Experts Concept & $(0.01,0.85-1.0,0.01$, & $(0.01,0.01$, & \\
& $0.01)$ & $0.01)$ & $(0.01,0.01,0.01$, \\
& $(0.01,0.95,0.01$, & $(0.01,0.01,0.01$, & $0.01)$ \\
\hline Oracle & $0.01)$ & $0.01)$ & $(1.0,0.01,0.01,0.01)$ \\
\hline Consumers & $(0.85-1.0,0.01,0.01$, & $(0.01,0.01,0.01$, & \\
Normal & $0.01)$ & $0.01)$ & $(1.0,0.65,0.01,0.01)$ \\
\hline Consumers & $(0.85-1.0,0.01,0.01$, & $(0.01,0.01,0.01$, & \\
Concept & $0.01)$ & $0.01)$ & \\
\hline
\end{tabular}

Oracle Concept Knowledge: $0 \rightarrow 1$

Only experts have enough expertise to provide answers to consumer agents as (0.5$1.0,0.01,0.01,0.01) \oplus(0.85-1.0,0.01,0.01,0.01)$ gives between 0.25 and 0.5 , which is greater than the simulation parameter CAPABILITY_THRESHOLD_FOR_ANSWERING_QUESTIONER.

\section{A.2 Learning}

\begin{tabular}{|l|l|}
\hline Variable & Value(s) \\
\hline NUM_NEIGHBOR_SET_CHANGES & 20 \\
\hline NUM_QUESTIONS_PER_NEIGHBOR_SET & 5 \\
\hline WAITING_LIMIT_FOR_MESSAGES & 1000 \\
\hline MAXIMUM_REFERRAL_CHAIN_LENGTH & 5 \\
\hline MAXIMUM_PERCENTAGE_PERTURBATION_FOR_GENERATING_QUERY & 0.0 \\
\hline MAXIMUM_PERCENTAGE_PERTURBATION_FOR_GENERATING_ANSWER & 0.0 \\
\hline RATE_OF_LEARNING_INTERESTS & 0.5 \\
\hline RATE_OF_LEARNING_FROM_GOOD_ANSWERS ( $\alpha)$ & $0.05,0.15,0.25$ \\
\hline ALPHA $(\alpha)$ & 0.25 \\
\hline PERCENTAGE_DECAY_FOR_NO_ANSWER & 0.0 \\
\hline BETA ( $\beta)$ & 0.2 \\
\hline W & 0.5 \\
\hline CAPABILITY_THRESHOLD_FOR_EVALUATING_ANSWER & 0.3 \\
\hline CAPABILITY_THRESHOLD_FOR_ANSWERING_QUESTIONER & 0.2 \\
\hline CAPABILITY_THRESHOLD_FOR_REFERING_QUESTIONER_TO_NEIGHBOR & $0.1,0.2,0.3,0.4$, \\
\hline CAPABILITY_THRESHOLD_FOR_ASKING_NEIGHBOR & 0.5 \\
\hline NUM_NEIGHBOURS & 0.01 \\
\hline PERCENT_EXPERTS & 8 \\
\hline
\end{tabular}




\section{Agent Interest and Expertise Setup}

\begin{tabular}{|l|l|l|l|}
\hline Agent Type & Interest & Expertise & Target Expertise \\
\hline Experts Normal & $(0.85-1.0,0.01,0.01$, & $(0.5-1.0,0.01,0.01$, & $(1.0,0.01,0.01,0.01)$ \\
& $0.01)$ & $0.01)$ & \\
\hline Experts Concept & $(0.01,0.85-1.0,0.01$, & $(0.01,0.5-1.0,0.01$, & $(0.01,1.0,0.01,0.01)$ \\
& $0.01)$ & $0.01)$ & \\
\hline Oracle & $(0.01,0.95,0.01$, & $(0.95,0.01,0.01$, & $(0.01,0.01,0.01$, \\
& $0.01)$ & $0.01)$ & $0.01)$ \\
\hline Consumers & $(0.85-1.0,0.01,0.01$, & $(0.01,0.01,0.01$, & $(1.0,0.01,0.01,0.01)$ \\
Normal & $0.01)$ & $0.01)$ & \\
\hline Consumers & $(0.85-1.0,0.01,0.01$, & $(0.01,0.01,0.01$, & $(1.0,0.65,0.01,0.01)$ \\
Concept & $0.01)$ & $0.01)$ & \\
\hline
\end{tabular}

Oracle Concept Knowledge: $0 \rightarrow 1$

Only experts have enough expertise to provide answers to consumer agents as (0.5$1.0,0.01,0.01,0.01) \oplus(0.85-1.0,0.01,0.01,0.01)$ gives between 0.25 and 0.5 , which is greater than the simulation parameter CAPABILITY_THRESHOLD_FOR_ANSWERING_QUESTIONER. As agents learn however they may contain enough expertise to answer queries as well.

\section{A.3 Trust}

\begin{tabular}{|c|c|}
\hline Variable & Value(s) \\
\hline NUM_NEIGHBOR_SET_CHANGES & 100 \\
\hline NUM_QUESTIONS_PER_NEIGHBOR_SET & 5 \\
\hline WAITING_LIMIT_FOR_MESSAGES & 1000 \\
\hline MAXIMUM_REFERRAL_CHAIN_LENGTH & 5 \\
\hline MAXIMUM_PERCENTAGE_PERTURBATION_FOR_GENERATING_QUERY & 0.0 \\
\hline MAXIMUM_PERCENTAGE_PERTURBATION_FOR_GENERATING_ANSWER & 0.0 \\
\hline RATE_OF_LEARNING_INTERESTS & 0.5 \\
\hline RATE_OF_LEARNING_FROM_GOOD_ANSWERS $(\alpha)$ & 0.1 \\
\hline $\operatorname{ALPHA}\left(\alpha^{\prime}\right)$ & 0.25 \\
\hline PERCENTAGE_DECAY_FOR_NO_ANSWER & 0.0 \\
\hline BETA $(\beta)$ & 0.2 \\
\hline W & 0.5 \\
\hline CAPABILITY_THRESHOLD_FOR_EVALUATING_ANSWER & 0.3 \\
\hline CAPABILITY_THRESHOLD_FOR_ANSWERING_QUESTIONER & 0.2 \\
\hline CAPABILITY_THRESHOLD_FOR_REFERING_QUESTIONER_TO_NEIGHBOR & 0.1 \\
\hline CAPABILITY_THRESHOLD_FOR_ASKING_NEIGHBOR & 0.01 \\
\hline AUTOMATA_STATES & $5,10,15$ \\
\hline USE_TRUST & True \\
\hline MAX_STAGNATION_SYCLES $\left(\mathrm{S}_{\mathrm{c}}\right)$ & 5 \\
\hline MAX_HEALTH_READINGS_FOR_STAGNATION $\left(\mathrm{H}_{\mathrm{r}}\right)$ & 5 \\
\hline
\end{tabular}




\begin{tabular}{|l|l|}
\hline HEALTH_STAGNATION_THRESHOLD $\left(\mathrm{H}_{\mathrm{t}}\right)$ & 0.1 \\
\hline NUM_NEIGHBOURS & 8 \\
\hline PERCENT_EXPERTS & 10 \\
\hline
\end{tabular}

Agent Interest and Expertise Setup

\begin{tabular}{|l|l|l|l|}
\hline Agent Type & Interest & Expertise & Target Expertise \\
\hline Experts & $(0.01,0.01,0.01,0.01$, & $(0.01,0.01,0.01,0.01$, & $(0.01,0.01,0.01$, \\
& $0.85-1.0)$ & $0.5-1.0)$ & $0.01,1.0)$ \\
\hline Oracle & $(0.01,0.85-1.0,0.01$, & $(0.01,0.01,0.01$, & $(0.01,0.65,0.01$, \\
& $0.01,0.01)$ & $0.01)$ & $0.01,0.01)$ \\
\hline Consumers & $(0.85-1.0,0.01,0.01$, & $(0.01,0.01,0.01,0.01$, & $(1.0,0.01,0.01,0.01$, \\
& $0.01,0.01)$ & $0.01)$ & $0.65)$ \\
\hline
\end{tabular}

Useless Oracle Concept Knowledge: $0 \rightarrow 1,1 \rightarrow 0,1 \rightarrow 2,2 \rightarrow 0,2 \rightarrow 1,2 \rightarrow 3,3 \rightarrow 1$, $3 \rightarrow 4$

Only experts have enough expertise to provide answers to consumer agents as $(0.01$, $0.01,0.01,0.01,0.5-1.0) \oplus(0.01,0.01,0.01,0.85-1.0)$ gives between 0.22 and 0.44 , which is greater than the simulation parameter CAPABILITY_THRESHOLD_FOR_ANSWERING_QUESTIONER. As agents learn however they may contain enough expertise to answer queries as well.

\section{A.4 Transitivity}

\section{CRF Network Setup}

\begin{tabular}{|l|l|}
\hline Variable & Value(s) \\
\hline NUM_NEIGHBOR_SET_CHANGES & 10 \\
\hline NUM_QUESTIONS_PER_NEIGHBOR_SET & 10 \\
\hline WAITING_LIMIT_FOR_MESSAGES & 1000 \\
\hline MAXIMUM_REFERRAL_CHAIN_LENGTH & 5 \\
\hline MAXIMUM_PERCENTAGE_PERTURBATION_FOR_GENERATING_QUERY & 0.0 \\
\hline MAXIMUM_PERCENTAGE_PERTURBATION_FOR_GENERATING_ANSWER & 0.0 \\
\hline RATE_OF_LEARNING_INTERESTS & 0.5 \\
\hline RATE_OF_LEARNING_FROM_GOOD_ANSWERS $(\alpha)$ & 0.1 \\
\hline ALPHA ( $\alpha$ ) & 0.25 \\
\hline PERCENTAGE_DECAY_FOR_NO_ANSWER & 0.0 \\
\hline BETA (ß) & 0.2 \\
\hline W & 0.5 \\
\hline CAPABILITY_THRESHOLD_FOR_EVALUATING_ANSWER & 0.3 \\
\hline CAPABILITY_THRESHOLD_FOR_ANSWERING_QUESTIONER & 0.2 \\
\hline CAPABILITY_THRESHOLD_FOR_REFERING_QUESTIONER_TO_NEIGHBOR & 0.2 \\
\hline CAPABILITY_THRESHOLD_FOR_ASKING_NEIGHBOR & 0.01 \\
\hline AUTOMATA_STATES & 5 \\
\hline USE_TRUST & True \\
\hline MAX_STAGNATION_SYCLES (S) & 5 \\
\hline
\end{tabular}




\begin{tabular}{|l|l|}
\hline MAX_HEALTH_READINGS_FOR_STAGNATION $\left(\mathrm{H}_{\mathrm{r}}\right)$ & 5 \\
\hline HEALTH_STAGNATION_THRESHOLD $\left(\mathrm{H}_{\mathrm{t}}\right)$ & 0.1 \\
\hline NUM_NEIGHBOURS & $\mathbf{8}$ \\
\hline PERCENT_EXPERTS & 10 \\
\hline
\end{tabular}

\section{Agent Interest and Expertise Setup}

\begin{tabular}{|l|l|l|l|}
\hline Agent Type & Interest & Expertise & Target Expertise \\
\hline Expert Domain 1 & $(0.01,0.95,0.01$, & $(0.01,0.95,0.01$, & $(0.01,0.95,0.01$, \\
& $0.01)$ & $0.01)$ & $0.01)$ \\
\hline Expert Domain 2 & $(0.01,0.01,0.95$, & $(0.01,0.01,0.95$, & $(0.01,0.01,0.95$, \\
& $0.01)$ & $0.01)$ & $0.01)$ \\
\hline Oracle & $(0.95,0.01,0.01$, & $(0.95,0.01,0.01$, & $(0.01,0.01,0.01$, \\
& $0.01)$ & $0.01)$ & $0.01)$ \\
\hline Consumers & $(0.7-0.95,0.01,0.01$, & $(0.01,0.01,0.01$, & $(0.01,0.95,0.95$, \\
& $0.01)$ & $0.01)$ & $0.01)$ \\
\hline
\end{tabular}

Oracle Concept Knowledge: $0 \rightarrow 1,1 \rightarrow 2$

Only experts have enough expertise to provide answers to consumer agents as $(0.01$, $0.95,0.01,0.01) \oplus(0.01,0.95,0.01,0.01)$ and $(0.01,0.01,0.95,0.01) \oplus(0.01,0.01,0.95$, 0.01 ) give 0.475 , which is greater than the simulation parameter CAPABILITY_THRESHOLD_FOR_ANSWERING_QUESTIONER. As agents learn however they may contain enough expertise to answer queries as well.

\section{RF1 Network Setup}

\begin{tabular}{|l|l|}
\hline Variable & Value(s) \\
\hline NUM_NEIGHBOR_SET_CHANGES & 10 \\
\hline NUM_QUESTIONS_PER_NEIGHBOR_SET & 10 \\
\hline WAITING_LIMIT_FOR_MESSAGES & 1000 \\
\hline MAXIMUM_REFERRAL_CHAIN_LENGTH & 5 \\
\hline MAXIMUM_PERCENTAGE_PERTURBATION_FOR_GENERATING_QUERY & 0.0 \\
\hline MAXIMUM_PERCENTAGE_PERTURBATION_FOR_GENERATING_ANSWER & 0.0 \\
\hline RATE_OF_LEARNING_INTERESTS & 0.5 \\
\hline RATE_OF_LEARNING_FROM_GOOD_ANSWERS $(\alpha)$ & 0.1 \\
\hline ALPHA ( $\alpha$ ) & 0.25 \\
\hline PERCENTAGE_DECAY_FOR_NO_ANSWER & 0.0 \\
\hline BETA( $\beta)$ & 0.2 \\
\hline W & 0.5 \\
\hline CAPABILITY_THRESHOLD_FOR_EVALUATING_ANSWER & 0.3 \\
\hline CAPABILITY_THRESHOLD_FOR_ANSWERING_QUESTIONER & 0.2 \\
\hline CAPABILITY_THRESHOLD_FOR_REFERING_QUESTIONER_TO_NEIGHBOR & 0.2 \\
\hline CAPABILITY_THRESHOLD_FOR_ASKING_NEIGHBOR & 0.01 \\
\hline AUTOMATA_STATES & 5 \\
\hline USE_TRUST & True \\
\hline
\end{tabular}




\begin{tabular}{|l|l|}
\hline MAX_STAGNATION_SYCLES $\left(\mathrm{S}_{\mathrm{c}}\right)$ & 5 \\
\hline MAX_HEALTH_READINGS_FOR_STAGNATION $\left(\mathrm{H}_{\mathrm{r}}\right)$ & 5 \\
\hline HEALTH_STAGNATION_THRESHOLD $\left(\mathrm{H}_{\mathrm{t}}\right)$ & 0.1 \\
\hline NUM_NEIGHBOURS & 8 \\
\hline PERCENT_EXPERTS & 10 \\
\hline
\end{tabular}

\section{Agent Interest and Expertise Setup}

\begin{tabular}{|l|l|l|l|}
\hline Agent Type & Interest & Expertise & Target Expertise \\
\hline Expert Domain 1 & $(0.01,0.95,0.01$, & $(0.01,0.95,0.01$, & $(0.01,0.95,0.01$, \\
& $0.01)$ & $0.01)$ & $0.01)$ \\
\hline Consumers & $(0.01,0.7-0.95,0.01$, & $(0.01,0.01,0.01$, & $(0.01,0.95,0.95$, \\
& $0.01)$ & $0.01)$ & $0.01)$ \\
\hline
\end{tabular}

\section{RF2 Network Setup}

\begin{tabular}{|l|l|}
\hline Variable & Value(s) \\
\hline NUM_NEIGHBOR_SET_CHANGES & 10 \\
\hline NUM_QUESTIONS_PER_NEIGHBOR_SET & 10 \\
\hline WAITING_LIMIT_FOR_MESSAGES & 1000 \\
\hline MAXIMUM_REFERRAL_CHAIN_LENGTH & 5 \\
\hline MAXIMUM_PERCENTAGE_PERTURBATION_FOR_GENERATING_QUERY & 0.0 \\
\hline MAXIMUM_PERCENTAGE_PERTURBATION_FOR_GENERATING_ANSWER & 0.0 \\
\hline RATE_OF_LEARNING_INTERESTS & 0.5 \\
\hline RATE_OF_LEARNING_FROM_GOOD_ANSWERS $(\alpha)$ & 0.1 \\
\hline ALPHA ( $\left.\alpha^{\prime}\right)$ & 0.25 \\
\hline PERCENTAGE_DECAY_FOR_NO_ANSWER & 0.0 \\
\hline BETA (ß) & 0.2 \\
\hline W & 0.5 \\
\hline CAPABILITY_THRESHOLD_FOR_EVALUATING_ANSWER & 0.3 \\
\hline CAPABILITY_THRESHOLD_FOR_ANSWERING_QUESTIONER & 0.2 \\
\hline CAPABILITY_THRESHOLD_FOR_REFERING_QUESTIONER_TO_NEIGHBOR & 0.2 \\
\hline CAPABILITY_THRESHOLD_FOR_ASKING_NEIGHBOR & 0.01 \\
\hline AUTOMATA_STATES & 5 \\
\hline USE_TRUST & True \\
\hline MAX_STAGNATION_SYCLES (S ) & 5 \\
\hline MAX_HEALTH_READINGS_FOR_STAGNATION (H) $)$ & 5 \\
\hline HEALTH_STAGNATION_THRESHOLD (Ht) & 0.1 \\
\hline NUM_NEIGHBOURS & 8 \\
\hline PERCENT_EXPERTS & 10 \\
\hline
\end{tabular}

\section{Agent Interest and Expertise Setup}

\begin{tabular}{|l|l|l|l|}
\hline Agent Type & Interest & Expertise & Target Expertise \\
\hline Expert Domain 1 & $(0.01,0.01,0.95$, & $(0.01,0.95,0.01$, & $0.01,0.95,0.01$, \\
\hline
\end{tabular}




\begin{tabular}{|l|l|l|l|}
\hline & $0.01)$ & $0.01)$ & $0.01)$ \\
\hline Consumers & $(0.01,0.01,0.7-0.95$, & $(0.01,0.01,0.01$, & $(0.01,0.95,0.95$, \\
& $0.01)$ & $0.01)$ & $0.01)$ \\
\hline
\end{tabular}

\section{A.5 Context}

\begin{tabular}{|c|c|}
\hline Variable & Value(s) \\
\hline NUM_NEIGHBOR_SET_CHANGES & 12 \\
\hline NUM_QUESTIONS_PER_NEIGHBOR_SET & 10 \\
\hline WAITING_LIMIT_FOR_MESSAGES & 1000 \\
\hline MAXIMUM_REFERRAL_CHAIN_LENGTH & 5 \\
\hline MAXIMUM_PERCENTAGE_PERTURBATION_FOR_GENERATING_QUERY & 0.0 \\
\hline MAXIMUM_PERCENTAGE_PERTURBATION_FOR_GENERATING_ANSWER & 0.0 \\
\hline RATE_OF_LEARNING_INTERESTS & 0.5 \\
\hline RATE_OF_LEARNING_FROM_GOOD_ANSWERS $(\alpha)$ & 0.1 \\
\hline ALPHA $\left(\alpha^{\prime}\right)$ & 0.25 \\
\hline PERCENTAGE_DECAY_FOR_NO_ANSWER & 0.0 \\
\hline BETA $(\beta)$ & 0.2 \\
\hline $\mathrm{W}$ & 0.5 \\
\hline CAPABILITY_THRESHOLD_FOR_EVALUATING_ANSWER & 0.3 \\
\hline CAPABILITY_THRESHOLD_FOR_ANSWERING_QUESTIONER & 0.2 \\
\hline CAPABILITY_THRESHOLD_FOR_REFERING_QUESTIONER_TO_NEIGHBOR & 0.2 \\
\hline CAPABILITY_THRESHOLD_FOR_ASKING_NEIGHBOR & 0.01 \\
\hline AUTOMATA_STATES & 5 \\
\hline USE_TRUST & True \\
\hline MAX_STAGNATION_SYCLES $\left(\mathrm{S}_{\mathrm{c}}\right)$ & 5 \\
\hline MAX_HEALTH_READINGS_FOR_STAGNATION $\left(\mathrm{H}_{\mathrm{r}}\right)$ & 5 \\
\hline HEALTH_STAGNATION_THRESHOLD $\left(\mathrm{H}_{\mathrm{t}}\right)$ & 0.1 \\
\hline NUM_NEIGHBOURS & 8 \\
\hline PERCENT_EXPERTS & 10 \\
\hline
\end{tabular}

\section{Agent Interest and Expertise Setup}

\begin{tabular}{|l|l|l|l|}
\hline Agent Type & Interest & Expertise & Target Expertise \\
\hline Expert Domain 1 & $(0.01,0.95,0.01$, & $(0.01,0.95,0.01$, & $(0.01,0.95,0.01$, \\
& $0.01)$ & $0.01)$ & $0.01)$ \\
\hline Expert Domain 2 & $(0.01,0.01,0.95$, & $(0.01,0.01,0.95$, & $(0.01,0.01,0.95$, \\
& $0.01)$ & $0.01)$ & $0.01)$ \\
\hline Oracle 1 & $(0.01,0.95,0.01$, & $(0.95,0.01,0.01$, & $(0.01,0.95,0.01$, \\
& $0.01)$ & $0.01)$ & $0.01)$ \\
\hline Oracle 2 & $(0.01,0.01,0.95$, & $(0.95,0.01,0.01$, & $(0.01,0.01,0.95$, \\
& $0.01)$ & $0.01)$ & $0.01)$ \\
\hline Consumers & $(0.7-0.95,0.01,0.01$, & $(0.01,0.01,0.01$, & $(0.01,0.95,0.95$, \\
& $0.01)$ & $0.01)$ & $0.01)$ \\
\hline
\end{tabular}

Oracle 1 Concept Knowledge: $0 \rightarrow 1$ 
Oracle 1 Concept Knowledge: $0 \rightarrow 2$

Only experts have enough expertise to provide answers to consumer agents as $(0.01$, $0.95,0.01,0.01) \oplus(0.01,0.95,0.01,0.01)$ and $(0.01,0.01,0.95,0.01) \oplus(0.01,0.01,0.95$, 0.01 ) give 0.475 , which is greater than the simulation parameter CAPABILITY_THRESHOLD_FOR_ANSWERING_QUESTIONER. As agents learn however they may contain enough expertise to answer queries as well. 


\section{References}

1. Yolum, P., \& Singh, M. P. (2003). Emergent Properties of Referral Systems. Proceedings of the Second International Joint Conference on Autonomous Agents and Multiagent Systems , 592-599.

2. Ricardo, F. J. (2001). Hypertext and knowledge management. Proceedings of the 12th ACM conference on Hypertext and Hypermedia , 217-226.

3. Yuen, L., Chang, M., Lai, Y. K., \& Poon, C. K. (2004). Excalibur: A Personalized Meta Search Engine. In Proceedings of the 28th Annual International Computer Software and Applications Conference , 49-50.

4. Guha, R., McCool, R., \& Miller, E. (2003). Semantic Search. Proceedings of the 12th international conference on World Wide Web , 700-709.

5. Wilson, T. (1981). On user studies and information needs. Journal of Documentation, 3-15.

6. Nardi, B., Whittaker, S., \& Schwarz, H. (2000, April 5). It's Not What You Know, It's Who You Know: Work in the Information Age. Retrieved March 10, 2007, from firstmonday.org: http://www.firstmonday.org/issues/issue5_5/nardi/

7. Androutsellis-Theotokis, S., \& Spinellis, D. (2004). A Survey of Peer-to-Peer Content Distribution Technologies. ACM Computing Surveys , 335-371.

8. Freenet.org. (2007, April 22). The Freenet Project. Retrieved April 22, 2007, from The Freenet Project: http://freenetproject.org/ 
9. Wikipedia. (2007, April 11). Peer-to-peer - Wikipedia, the free encyclopedia. Retrieved April 12, 2007, from Wikipedia: http://en.wikipedia.org/wiki/ Peer_to_peer_network

10. Yolum, P. (2003). Properties of Referral Networks: Emergence of Authority and Trust [dissertation]. Department of Computer Science, North Caroline State University.

11. Yolum, P., \& Sensoy, M. (2006). A Context-Aware Approach for Service Selection Using Ontologies. 5th International Joint Conference on Autonomous Agents and Multiagent Systems (AAMAS) , 931-938.

12. Caravalho, M., Hewett, R., \& Canas, A. J. (2001). Enhancing Web Searches from Concept Map-based Knowledge Models. Pensacola, FL: Institute for Human \& Machine Cognition.

13. Marsh, S. P. (1994). Formalising Trust as a Computational Concept [dissertation]. Stirling: Department of Computing Science and Mathematics, University of Stirling.

14. Blachman, N., \& Peek, J. (2007, February 2). How Google Works. (GoogleGuide) Retrieved April 20, 2007, from Googleguide Web site: http://www.googleguide.com/google_works.html

15. Page, L., Brin, S., Motwani, R., \& Winograd, T. (1998). The PageRank Citation Ranking: Bringing Order to the Web. Stanford: Stanford Digital Library Technologies Project. 
16. Cutts, M. (2007, January 25). How does Google collect and rank results? (Google) Retrieved April 26, 2007, from Google: http://www.google.com/librariancenter/articles/0512_01.html

17. Abawajy, J. H., \& Hu, M. J. (2005). A New Internet Meta-Search Engine and Implementation. The 3rd ACS/IEEE International Conference on Computer Systems and Applications , 103-111.

18. Barker, J. (2006, June 19). Meta-Search Engines. (University of California) Retrieved April 12, 2007, from University of California Web site: http://www.lib.berkeley.edu/TeachingLib/Guides/Internet/MetaSearch.htm 1

19. Klampanos, I. A. (2002, 6 10). Infrasearch. (University of Glasgow) Retrieved 4 14, 2007, from University of Glasgow - Computing Science: http://www.dcs.gla.ac.uk/ iraklis/fyp_report/node19.html

20. Novak, J. D., \& Gowin, D. B. (1984). Learning How to Learn. Cambridge, N.Y., United States: Cambridge University Press.

21. Guarino, N., and Giaretta, P. (1995). “Ontologies and Knowledge bases: towards a terminological clarification", Towards Very Large Knowledge Bases: Knowledge Building and Knowledge Sharing. N. Mars, IOS Press: 2532

22. S.E. Middleton, D.C. De Roure, N.R. Shadbolt (2002). Capturing Knowledge of User Preferences: ontologies on recommender systems. First international conference on Knowledge Capture 2001. 
23. Goldberg, D., Nichols, D., M. Oki, B., \& Terry, D. (1992). Using collaborative filtering to weave an information tapestry. Communications of the ACM, 6170.

24. Resnick, P., \& Varian, H. R. (1997). Recommender systems. Communications of the ACM, 56-58.

25. Blanzieri, E., \& Giorgini, P. (2000). From Collaborative Filtering to Implicit Culture: a general agent-based framework. In Proceedings of the Workshop on Agents and Recommender Systems.

26. Birukov, A., Blanzieri, E., \& Giorgini, P. (2005). Implicit: An Agent-Based Recommendation System for Web Search. Proceedings of the 4th International Conference on Autonomous Agents and Multi-Agent Systems, 618-624.

27. Blanzieri, E., Giorgini, P., Giunchiglia, F., \& Zanoni, C. (2004). Implicit culturebased personal agents for knowledge management. Lecture Notes in Artificial Intelligence, 245-261.

28. Blanzieri, E., Giorgini, P., Massa, P., \& Recla, S. (2000). Implicit Culture for Multi-agent Interaction Support. Lecture Notes in Computer Science, 27-40.

29. Lehikoinen, J., Salminen, I., Aaltonen, A., Huuskonen, P., \& Kaario, J. (2006). Meta-searches in peer-to-peer networks. Personal and Ubiquitous Computing , 357-367.

30. Montaner, M., López, B., \& Lluís de la Rosa, J. (2002). Opinion-Based Filtering through Trust. Lecture Notes In Computer Science, 164 - 178. 
31. Berners-Lee, T., Hendler, J., \& Lassila, O. (2001). The semantic web. Scientific American.

32. Guha, R., \& McCool, R. (2003, 02 07). TAP - An Application Framework for Building the Semantic Web. Retrieved 04 10, 2007, from TAP - An Application Framework for Building the Semantic Web: http://www.w3.org/2002/05/tap/

33. Kautz, H., Selman, B., \& Shah, M. (1997). The hidden web. The American Association for Artificial Intelligence , 27-36.

34. Yolum, P., \& Singh, M. P. (2003). Dynamic Communities in Referral Networks. Web Intelligence and Agent Systems , 105-116.

35. Yolum, P., \& Singh, M. P. (2003). Self-Organizing Referral Networks: A Process View of Trust and Authority. AAMAS Workshop on Engineering SelfOrganising Applications, 195-211.

36. Yolum, P., Udupi, Y., \& Singh, M. P. (2003). Trustworthy Service Caching: Cooperative Search in P2P Information Systems. AAMAS Workshop on Agent-Oriented Information Systems, 33-46.

37. Granovetter, M. (1973). The strength of weak ties. American Journal of Sociology, 1360-1380.

38. Yu, B., Singh, M. P., \& Venkatraman, M. (2001). Community-based Service Location. Communications of the ACM, 49-54.

39. Yu, B., Venkatraman, M., \& Singh, M. P. (2003). An Adaptive Social Network for Information Access: Theoretical and Experimental Results. Journal of the Applied Artificial Intelligence, 21-38. 
40. Yolum, P., \& Singh, M. P. (2004). Achieving Trust via Service Graphs. International Conference on Cooperative Information Systems, 509-525.

41. Yu, B. (2001). Emergence and Evolution of Agent-based Referral Networks [dissertation]. Department of Computer Science, North Caroline State University.

42. Marcgiori, M. (1997). The quest for correct information on the Web: Hyper search engines. Computer Networks and ISDN Systems , 1225-1235.

43. Wu, B., \& Davison, B. D. (2005). Identifying Link Farm Spam Pages. Special interest tracks and posters of the 14th international conference on World Wide Web , 820-829.

44. Yu, B., \& Singh, M. P. (2003). Searching Social Networks. Proceedings of the Second International Joint Conference on Autonomous Agents and MultiAgent Systems , 65-72.

45. Salton, G., \& McGill, M. J. (1983). An Introduction to Modern Information Retrieval. New York: McGraw-Hill.

46. Yolum, P., \& Singh, M. P. (2004). Engineering Self-Organizing Referral Networks for Trustworthy Service Selection. IEEE Transactions on systems, man, and cybernetics, 1-10.

47. Yolum, P., \& Singh, M. P. (2002). An Agent-Based Approach for Trustworthy Service Location. Lecture Notes in Artificial Intelligence, 45-56.

48. Brin, S., \& Page, L. (1998). The anatomy of a large scale hypertextual Web search engine. Computer Networks and ISDN Systems , 107-117. 
49. Cormen, T. H., Leiserson, C. E., Rivest, R. L., \& Stein, C. (2001). Introduction to Algorithms Second Edition. Cambridge: McGraw-Hill.

50. Gerck, E. (2002). Trust as Qualified Reliance on Information. Ewing: COOK Network Consultants.

51. Kaelbling, L. P., Littman, M. L., \& Moore, A. W. (1996). Reinforcement Learning: A Survey. Journal of Artificial Intelligence Research , 237-285. 